DESY $94-018$

ISSN $0418-9833$

February 1994

\title{
PATH INTEGRAL DISCUSSION FOR SMORODINSKY-WINTERNITZ POTENTIALS: I. TWO- AND THREE DIMENSIONAL EUCLIDEAN SPACE
}

\author{
C. Grosche* \\ II. Institut für Theoretische Physik \\ Universität Hamburg, Luruper Chaussee 149 \\ 22761 Hamburg, Germany \\ G. S. Pogosyan** and A. N. Sissakian** \\ Laboratory of Theoretical Physics \\ Joint Institute for Nuclear Research (Dubna) \\ 141980 Dubna, Moscow Region, Russia
}

\begin{abstract}
Path integral formulations for the Smorodinsky-Winternitz potentials in two- and threedimensional Euclidean space are presented. We mention all coordinate systems which separate the Smorodinsky-Winternitz potentials and state the corresponding path integral formulations. Whereas in many coordinate systems an explicit path integral formulation is not possible, we list in all soluble cases the path integral evaluations explicitly in terms of the propagators and the spectral expansions into the wave-functions.
\end{abstract}

\footnotetext{
* Supported by Deutsche Forschungsgemeinschaft under contract number GR 1031/2-1.

** Supported by Heisenberg-Landau program.
} 



\section{Introduction.}

In the study of the Kepler problem and the harmonic oscillator it turns out that they possess properties making them of special interest, for instance, all finite classical trajectories are closed and all energy eigen-values are multiply degenerated. These properties point beyond their specific shape and give rise to ask about their "hidden" or "dynamical" group structure, namely whether it is possible to distort them in a systematic way, and at the same time keep these properties. Furthermore, do they belong to a class of potential problems which have similar properties?

For instance, as has been known for a long time, at the basis of the specific properties of the hydrogen atom lies its $\mathrm{O}(4)$ symmetry, or to be more precise, the dynamical group $\mathrm{O}(4)$ for the discrete spectrum and the Lorentz group $\mathrm{O}(3,1)$ for the continuous spectrum. All such systems have the particular property that all the energy-levels of the system are organized in representations of the non-invariance group which contain representations of the dynamical subgroup realized in terms of the wave-functions of these energy-levels [42]. In the case of the hydrogen it enabled Pauli [112] and Fock [39] to solve the quantum mechanical Kepler problem without explicitly solving the Schrödinger equation. Actually, the algebra of the dynamical symmetry of the hydrogen atom turns out to be a centerless twisted Kac-Moody algebra [23].

Smorodinsky, Winternitz et al. started a systematic search to find and classify potential problems in two and three dimensions which can be seen as non-central generalizations of the Coulomb-, the harmonic oscillator and radial barrier potentials. The classification scheme starts from the consideration in which way integrals of motion (in classical mechanics), respectively additional operators corresponding to these integrals of motion which commute with the Hamiltonian, are related to the separability of the potential problem in more than one coordinate system. In two dimensions 442 it turns out that there are four potentials of the sought type which all have three constants of motion (including energy), i.e. there are two more operators commuting with the Hamiltonian. Smorodinsky, Winternitz et al. extended their investigations in a classical paper [93] to three dimensions by listing all potentials which separate in more than one coordinate system. However, in three dimensions one must distinguish two cases, namely minimally super-integrable systems, where four, and maximally super-integrable systems, where five functionally independent integrals of motion exist.

Actually in $D$ dimensions a system is called minimally super-integrable if it has $2 D-2$ functionally independent integrals of motions, and it is called maximally super-integrable if it has $2 D-1$ functionally independent integrals of motion [0, 64]. A discussion of the two-dimensional case is due to e.g. Winternitz et al. [129], and a detailed study of the three-dimensional case can be found in [35, 82]. Moreover, the Smorodinsky-Winternitz potentials allow a generalization to $D$ dimensions and are then also maximally super-integrable 36].

In the three-dimensional Euclidean space there are eleven coordinate systems which separate the free Hamiltonian [74, 101, 105]. Each potential must now be analysed according to its separability in these coordinate systems. Because each potential can be rotated and translated with respect to itself, one has to look for the equivalence classes among them. As it turns out there are eight minimally super-integrable (including a ring-shaped oscillator and the Hartmann potential) and five maximally super-integrable (including harmonic oscillator and Coulomb) potentials. Evans [35] presented a list of these potentials including the corresponding integrals of motion and (almost) all separating coordinate systems. The group structure of the twodimensional potentials was given in [42], for the Hartmann potential by Gerry [46] and Granovsky et al. [50], for the ring-shaped oscillator by Quesne [116] and Zhedanov [131], and the harmonic oscillator including $1 / x^{2}$-terms (a Smorodinsky-Winternitz potential, see below) was analysed by Evans [35]. 
The purpose of this paper is to give a path integral discussion of all Smorodinsky-Winternitz potentials in two- and three-dimensional Euclidean space. This includes the statement of the propagator (the time-evolution kernel), respectively, if only available, the corresponding Green function, and the spectral expansion into wave-functions and energy-levels. In the discussion of the two-dimensional Smorodinsky-Winternitz potentials the evaluation will be somewhat more elaborated in order to demonstrate the techniques. Of particular importance will be the basic path integral solutions of the radial harmonic oscillator by Peak and Inomata [113] (compare also Duru [29] and Goovaerts [48]), and of the Pöschl-Teller potential by Böhm and Junker [6] which is based on the $\mathrm{SU}(2)$ path integral (compare also Duru [28], and Inomata and Wilson [72]). All these path integral solutions are now well-known in the literature and will not be repeated here.

One may ask, if it is possible to analyse the path integral of a potential problem in terms of its dynamical symmetry group [70, 71]. In order to look at such a path integral formulation we consider the Lagrangian $\mathcal{L}(\vec{x}, \dot{\vec{x}})-V(\vec{x})\left(\vec{x} \in \mathbb{R}^{p+q}\right)$ as formulated, say, in a not-necessarily positive definite space with signature

$$
\left(g_{a b}\right)=\operatorname{diag}(\underbrace{+1, \ldots,+1}_{p \text { times }}, \underbrace{-1, \ldots,-1}_{q \text { times }}) .
$$

One introduces, say, polar coordinates $x_{\nu}=r e_{\nu}\left(\theta_{1}, \ldots, \theta_{p+q-1}\right)(\nu=1, \ldots, p+q)$, where the $\vec{e}$ are unit vectors in some suitable chosen (timelike, spacelike or lightlike) set [ [6]. One then expresses the Lagrangian in terms of these polar coordinates and seeks for an expansion of the quantity $\mathrm{e}^{z\left(\vec{e}_{1} \cdot \vec{e}_{2}\right)}$ expressed in terms of group elements $g_{1}, g_{2}$. If this is possible one can reexpress the path integration of the coordinates $\vec{x}$ into a path integration over group elements $g$ yielding [6, 26, 27, 96, 114]

$$
\begin{aligned}
\int_{\vec{x}\left(t^{\prime}\right)=\vec{x}^{\prime}}^{\vec{x}\left(t^{\prime \prime}\right)=\vec{x}^{\prime \prime}} \mathcal{D} \vec{x}(t) \exp \left[\frac{\mathrm{i}}{\hbar} \int_{t^{\prime}}^{t^{\prime \prime}} \mathcal{L}(\vec{x}, \dot{\vec{x}}) d t\right] & \mapsto \int d E_{\lambda} d_{\lambda} \sum_{m n} \hat{f}_{m n}^{\lambda} D_{m n}^{\lambda}\left(g^{\prime-1} g^{\prime \prime}\right) \\
& =\int d E_{\lambda} d_{\lambda} \sum_{m n} \hat{f}_{m n}^{\lambda} \sum_{k} D_{m, k}^{\lambda *}\left(g^{\prime}\right) D_{m, k}^{\lambda}\left(g^{\prime \prime}\right) .
\end{aligned}
$$

Here $\hat{f}_{m n}^{\lambda}$ is defined via the Fourier transformation

$$
f(g)=\int d E_{\lambda} d_{\lambda} \sum_{m n} \hat{f}_{m n}^{\lambda} D_{m n}^{\lambda}(g), \quad \hat{f}_{m n}^{\lambda}=\int_{G} f(g) D_{m n}^{\lambda *}\left(g^{-1}\right) d g
$$

and $d g$ is the invariant group (Haar) measure. $\int d E_{\lambda}$ stands for a Lebesque-Stieltjes integral to include discrete $\left(\int d E_{\lambda} \rightarrow \sum_{\lambda}\right)$ as well as continuous representations. $\int d E_{\lambda}$ is to be taken over the complete set $\{\lambda\}$ of class one representations. $d_{\lambda}$ denotes (in the compact case) the dimension of the representation and we take

$$
d_{\lambda} \int_{G} D_{m n}^{\lambda}(g) D_{m^{\prime} n^{\prime}}^{\lambda^{\prime}}(g) d g=\delta\left(\lambda, \lambda^{\prime}\right) \delta_{m, m^{\prime}} \delta_{n, n^{\prime}}
$$

as a definition for $d_{\lambda} . \delta\left(\lambda, \lambda^{\prime}\right)$ can denote a Kronecker delta, respectively, a $\delta$-function, depending whether the quantity $\lambda$ is a discrete or continuous parameter. We have furthermore used the group (composition) law

$$
D_{m n}^{\lambda}\left(g_{a}^{-1} g_{b}\right)=\sum_{k} D_{k n}^{\lambda *}\left(g_{a}\right) D_{k m}^{\lambda}\left(g_{b}\right)
$$

Choosing a basis $\{\vec{b}\}$ in the relevant Hilbert space fixes the matrix elements $D_{m n}^{\lambda}$ through $D_{m n}^{\lambda}=\left(D^{\lambda}(g) b_{m}, b_{n}\right)$ of the representation $D^{\lambda}(g)$ of the group. In particular the $D_{0 m}^{\lambda}$ are called 
associated spherical harmonics, and the $D_{00}^{\lambda}$ are the zonal harmonics. These spherical functions are eigen-functions of the corresponding Laplace-Beltrami operator on a, say, homogeneous space, and the entire Hilbert space is spanned by a complete set of associated spherical functions $D_{0 m}^{\lambda}$ (Gelfand et al. [43, 44] and Vilenkin [126]). In [58] a systematic study was presented of path integrals of the free motion in two- and three-dimensional spaces of constant curvature, i.e. on homogeneous spaces.

The Smorodinsky-Winternitz potentials can also be discussed in the context of a group path integration, provided the dynamical "hidden" group structure behind the potential problem is known, and the wave-functions and the energy-levels follow from the representations of the group [70, 71]. However, things are not as simple as for the free motion because we have not a problem of quantum motion on a homogeneous space. Whereas in many coordinate system realizations we can actually evaluate the path integral, there remain several ones, where a direct path integral evaluation is not possible because we obtain quartic, sextic, complicated Mathieulike path integral problems, where no solution is known. Also a consideration along the lines of "quasi-exactly solvable path integrals" [89] can not be applied in an obvious way. However, sometimes the zero-energy Green's function can be evaluated. As we will see, the technique of separation of variables in the path integral [55] will give us a convenient tool to exploit the actually symmetry structure in such a way that we can systematically reduce the number of degrees of freedom by using the appropriate path integral identities.

We will therefore provide the path integral formulations of all Smorodinsky-Winternitz potentials in two- and three-dimensional Euclidean space. Some can only be stated, others can be explicitly solved. We find that each of the potentials can be at least solved in one coordinate system. The identities arising from the relations between the various formulations serve as a tool to analyse the "hidden" dynamical group structure, however, only in an implicit way. The representations of the group in question where a path integral is not explicitly soluble remain still unknown. Furthermore, the explicit spectral expansions corresponding to various coordinate systems allow to study the interbasis expansions of the problem. An example of particular importance is the two-dimensional hydrogen atom [24, 95, respectively the ring-shaped oscillator [90], and the Hartmann potential [90, 131].

The knowledge of soluble examples and their relation with each other, in particular here the Smorodinsky-Winternitz potentials, is of great value in many branches of theoretical physics. For instance, the Hartmann system [63, 79, 81] with either an oscillator or an Coulomb interaction term appears in the study of molecule physics (modeling ring-shaped molecules like the benzene molecule), in the study of the Aharonov-Bohm effect [1, 80], for path integral studies c.f. e.g. (Bernido and Inomata [4], Cheng [16], Chetouani et al. 18], Gerry and Singh [45], Hoang and Giang [88], Sökmen [117], Tanikella and Inomata [123], and Wiegel [127]), and for a more recent discussion of a deformed generalization [76]. Evans [35] also mentioned cases in celestical mechanics (e.g. an artificial satellite orbiting an oblate planet, dynamics of elliptical galaxies). The consideration of the Smorodinsky-Winternitz potentials in spaces of constant (positive and negative) curvature models a space-time of closed, respectively open universes, c.f. the Kepler problem and the Higgs oscillator problem (Granovsky et al. [51], Higgs [65], Infeld [67], Leemon [87], Otchik and Red'kov [108], Pogosyan et al. [115], Schrödinger [121], and Steveson [120], and path integral discussions c.f. Barut et al. [2, 34, and Grosche [54]). Path integral discussions of Smorodinsky-Winternitz potentials in space of constant positive and negative curvature will be given in forthcoming publications. Starting from a particular exactly soluble example one can perturb it by a further (vector or scalar) potential which is not destroying separability in a conveniently chosen coordinate system [93], one can expand about the exact solution of the unperturbed one, hence giving a perturbation series in, say, either powers of the coupling parameter (e.g. 100), respectively in powers of $\hbar$. The latter then gives a "pseudo" semi-classical expansion á la DeWitt-Morette [99]-103]. 
Our work can be seen as a generalization and extension of the earlier work of Carpio-Bernido et al. [10]-[14]. They discussed only some of the Smorodinsky-Winternitz potentials and they restricted themselves only to a spherical polar coordinate path integral discussion in $E^{(3)}$, the three-dimensional Euclidean space. Their results are, of course, included in our discussion. They also did not discuss a classification scheme of the potentials. In particular, all our path integral evaluations in parabolic coordinates will be new path integral solutions, including the expansion into discrete and continuous wave-functions (if present). The path integral solutions of the two-dimensional Smorodinsky-Winternitz potential $V_{4}$ and three-dimensional SmorodinskyWinternitz potential $V_{3}$ are entirely new (in Refs. [10]-14] special cases of the latter have been discussed). We therefore give a combined presentation of henceforth solutions which have been scattered around in the literature and we also give all admissable solutions of each potential problem.

Our paper will be organized as follows: In the next Section we will list for completeness the coordinate systems in two- and three-dimensional flat space which separate the free Helmholtz, respectively Schrödinger equation. Also a separation formula for path integrals will be stated.

In the third Section we discuss the Smorodinsky-Winternitz potentials in two and three dimensions, respectively. This includes the formulation of the path integral in the various separating coordinate systems, and if possible via the basic path integrals, its solution in terms of the propagator, respectively the Green functions, and its spectral expansion. In particular, we will make frequently use of the separation technique in path integrals as developed in [55], and will not write down explicitly each step in the calculation of the examples of the explicitly soluble Smorodinsky-Winternitz potentials. In our path integral solutions we will state the Feynman kernels as explicitly as possible, and their spectral expansions according to

$$
\begin{aligned}
K\left(\vec{x}^{\prime \prime}, \vec{x}^{\prime} ; T\right)= & \int d E_{\lambda} \Psi_{\lambda}^{*} \lambda\left(\vec{x}^{\prime}\right) \Psi_{\lambda}\left(\vec{x}^{\prime \prime}\right) \mathrm{e}^{-\mathrm{i} E_{\lambda} T / \hbar} \\
= & \left\{\begin{array}{l}
\sum_{n} \Psi_{n}\left(\vec{x}^{\prime}\right) \Psi_{n}\left(\vec{x}^{\prime \prime}\right) \mathrm{e}^{-\mathrm{i} E_{n} T / \hbar} \\
\int d p \Psi_{p}\left(\vec{x}^{\prime}\right) \Psi_{p}\left(\vec{x}^{\prime \prime}\right) \mathrm{e}^{-\mathrm{i} \hbar p^{2} T / 2 M} \\
\sum_{n} \Psi_{n}\left(\vec{x}^{\prime}\right) \Psi_{n}\left(\vec{x}^{\prime \prime}\right) \mathrm{e}^{-\mathrm{i} E_{n} T / \hbar}+\int d p \Psi_{p}^{*}\left(\vec{x}^{\prime}\right) \Psi_{p}\left(\vec{x}^{\prime \prime}\right) \mathrm{e}^{-\mathrm{i} \hbar p^{2} T / 2 M},
\end{array}\right.
\end{aligned}
$$

whether the spectrum is purely discrete, purely continuous, or discrete and continuous. In these cases, where only the (energy-dependent) Green function is available, the spectral expansions will be presented similarly.

The fourth Section is devoted to a summary and a discussion of our results.

\section{Coordinate Systems in Euclidean Space and the Path Integral.}

\subsection{Coordinate Systems in Euclidean Space.}

2.1.1. Coordinate Systems in D Dimensions. In D-dimensional Euclidean space we have the coordinate system corresponding to the elliptic coordinates

$$
\begin{aligned}
& x_{j}^{2}=\frac{\prod_{i=1}^{D}\left(\rho_{i}-e_{j}\right)}{\prod_{i \neq j}^{D}\left(e_{i}-e_{j}\right)}, \quad(j=1, \ldots, D) \\
& e_{1}<\rho_{1}<e_{2}<\ldots<e_{D}<\rho_{D} .
\end{aligned}
$$

This is the most general coordinate system possible, and all other coordinate systems are included as degenerations. In the following we will explicitly state the coordinate systems in 
two- and three-dimensional flat space $E^{(2)}$ and $E^{(3)}$ which separate the Helmholtz, respectively the Schrödinger equation. We rewrite the Hamiltonian, respectively the Laplacian $\Delta_{L}=$ $g^{-1 / 2} \partial_{a} g^{a b} g^{1 / 2} \partial_{b}$ in terms of the corresponding momentum operators $p_{a}=-\mathrm{i} \hbar g^{-1 / 4} \partial_{a} g^{1 / 4}=$ $-\mathrm{i} \hbar\left(\partial_{a}+\Gamma_{a} / 2\right)\left(\Gamma_{a}=\partial_{a} \ln \sqrt{g}, g \operatorname{det}\left(g_{a b}\right)\right)$, where we choose a specific ordering prescription called product ordering [52]. Here it is assumed that $g_{a b}=h_{a c} h_{c b}, g^{a b}=h^{a c} h^{c b}$, the $h_{a c}$ being vielbeins, such that we can write $H=-\frac{\hbar^{2}}{2 M} \Delta_{L}=\frac{1}{2 M} h^{a c} p_{a} p_{b} h^{c b}+\Delta V_{P F}$ with the quantum potential $\Delta V_{P F}$ given by

$$
\Delta V_{P F}=\frac{\hbar^{2}}{8 M}\left[g^{a b} \Gamma_{a} \Gamma_{b}+2\left(g^{a b} \Gamma_{b}\right)_{, b}+g_{, a b}^{a b}\right]+\frac{\hbar^{2}}{8 M}\left(2 h^{a c} h_{, a b}^{b c}-h_{, a}^{a c} h_{, b}^{b c}-h_{, b}^{a c} h_{, a}^{b c}\right) .
$$

The potentials which may be added without spoiling the separability were derived by Eisenhardt [32] and Smorodinsky and Winternitz et al. [42, 93].

\subsubsection{Coordinate Systems in Two Dimensions.}

Cartesian Coordinates. We consider the usual cartesian coordinates $(x, y)=\vec{x} \in \mathbb{R}^{2} \equiv E^{(2)}$. The metric is given by $\left(g_{a b}\right)=\mathbb{1}_{2}$ and for the momentum operators we have $p_{x}=-\mathrm{i} \hbar \partial_{x}$, $p_{y}=-\mathrm{i} \hbar \partial_{y}$. Therefore:

$$
-\frac{\hbar^{2}}{2 M} \Delta_{E^{(2)}}=-\frac{\hbar^{2}}{2 M}\left(\frac{\partial^{2}}{\partial x^{2}}+\frac{\partial^{2}}{\partial y^{2}}\right)=\frac{1}{2 M} \vec{p}^{2}
$$

The most general potential separable in cartesian coordinates has the form

$$
V(\vec{x})=u(x)+v(y) .
$$

Polar Coordinates. Next, we consider two-dimensional polar coordinates

$$
\begin{array}{ll}
x=\rho \cos \phi, & \rho>0, \\
y=\rho \sin \phi, & 0 \leq \phi<2 \pi .
\end{array}
$$

The metric is given by $\left(g_{a b}\right)=\operatorname{diag}\left(1, \rho^{2}\right)$, and the momentum operators have the form

$$
p_{\rho}=\frac{\hbar}{\mathrm{i}}\left(\frac{\partial}{\partial \rho}+\frac{1}{2 \rho}\right), \quad p_{\phi}=\frac{\hbar}{\mathrm{i}} \frac{\partial}{\partial \phi} .
$$

This gives for the Hamiltonian

$$
-\frac{\hbar^{2}}{2 M} \Delta_{E^{(2)}}=-\frac{\hbar^{2}}{2 M}\left(\frac{\partial^{2}}{\partial \rho^{2}}+\frac{1}{\rho} \frac{\partial}{\partial \rho}+\frac{1}{\rho^{2}} \frac{\partial^{2}}{\partial \phi^{2}}\right)=\frac{1}{2 M}\left(p_{\rho}^{2}+\frac{1}{\rho^{2}} p_{\phi}^{2}\right)-\frac{\hbar^{2}}{8 M \rho^{2}} .
$$

A separable potential has the form

$$
V(\vec{x})=u(\rho)+\frac{1}{\rho^{2}} v(\phi) .
$$

Elliptic Coordinates. Third, we consider the coordinate system

$$
\begin{array}{ll}
x=d \cosh \xi \cos \eta, & \xi>0, \\
y=d \sinh \xi \sin \eta, & -\pi<\eta \leq \pi,
\end{array}
$$

and $d=R / 2$, where $R$ is the interfocus distance. The metric is $\left(g_{a b}\right)=d^{2}\left(\sinh ^{2} \xi+\sin ^{2} \eta\right) \mathbb{1}_{2}$ and we obtain for the momentum operators

$$
p_{\xi}=\frac{\hbar}{\mathrm{i}}\left(\frac{\partial}{\partial \xi}+\frac{\sinh \xi \cosh \xi}{\sinh ^{2} \xi+\sin ^{2} \eta}\right), \quad p_{\eta}=\frac{\hbar}{\mathrm{i}}\left(\frac{\partial}{\partial \eta}+\frac{\sin \eta \cos \eta}{\sinh ^{2} \xi+\sin ^{2} \eta}\right) .
$$


Consequently we have for the Hamiltonian

$$
\begin{aligned}
-\frac{\hbar^{2}}{2 M} \Delta_{E^{(2)}} & =-\frac{\hbar^{2}}{2 M d^{2}\left(\sinh ^{2} \xi+\sin ^{2} \eta\right)}\left(\frac{\partial^{2}}{\partial \xi^{2}}+\frac{\partial^{2}}{\partial \eta^{2}}\right) \\
& =\frac{1}{2 M d^{2}}\left(\sinh ^{2} \xi+\sin ^{2} \eta\right)^{-1 / 2}\left(p_{\xi}^{2}+p_{\eta}^{2}\right)\left(\sinh ^{2} \xi+\sin ^{2} \eta\right)^{-1 / 2} .
\end{aligned}
$$

Replacing $x \mapsto x=d(\cosh \xi \cos \eta+1)$ yields a coordinate system which we call in the following elliptic II coordinates [95]. The elliptic coordinate system is useful in the study of two center systems. Furthermore, an additional parameter is introduced, the interfocus distance $R$. This distinguishes it from the other two-dimensional coordinate systems which can be obtained from the elliptic one by considering specific limits. In particular $R \rightarrow 0$ reproduces polar coordinates, and $R \rightarrow \infty$ cartesian coordinates. In the case of the elliptic II systems, $R \rightarrow \infty$ reproduces parabolic coordinates (see below).

Note $\Delta V_{P F}=0$ which is a peculiarity of two dimensions with metric $\propto \mathbb{1}$, c.f. (10). A potential separable in these coordinates reads

$$
V(\vec{x})=\frac{u(\cosh \xi)+v(\cos \eta)}{\sinh ^{2} \xi+\sin ^{2} \eta} .
$$

Parabolic Coordinates. Finally, we consider the coordinate system

$$
x=\frac{1}{2}\left(\eta^{2}-\xi^{2}\right), \quad y=\xi \eta, \quad \xi \in \mathbb{R}, \eta>0
$$

(alternatively $\xi>0, \eta \in \mathbb{R}[97])$. We have $\left(g_{a b}\right)=\left(\xi^{2}+\eta^{2}\right) \mathbb{1}_{2}$, and consequently for the momentum operators

$$
p_{\xi}=\frac{\hbar}{\mathrm{i}}\left(\frac{\partial}{\partial \xi}+\frac{\xi}{\xi^{2}+\eta^{2}}\right), \quad p_{\eta}=\frac{\hbar}{\mathrm{i}}\left(\frac{\partial}{\partial \eta}+\frac{\eta}{\xi^{2}+\eta^{2}}\right) .
$$

This gives for the Hamiltonian

$$
-\frac{\hbar^{2}}{2 M} \Delta_{E^{(2)}}=-\frac{\hbar^{2}}{2 M\left(\xi^{2}+\eta^{2}\right)}\left(\frac{\partial^{2}}{\partial \xi^{2}}+\frac{\partial^{2}}{\partial \eta^{2}}\right)=\frac{1}{2 M}\left(\xi^{2}+\eta^{2}\right)^{-1 / 2}\left(p_{\xi}^{2}+p_{\eta}^{2}\right)\left(\xi^{2}+\eta^{2}\right)^{-1 / 2} .
$$

These coordinates have been used in the literature to discuss the two-dimensional "Coulombproblem", c.f. [31, 42, 57, 68]. The transformation $(x, y) \mapsto(\xi, \eta)$ actually is the two-dimensional realization of the Levi-Civita transformation. A separable potential has the form

$$
V(\vec{x})=\frac{u(\xi)+v(\eta)}{\xi^{2}+\eta^{2}}
$$

\subsubsection{Coordinate Systems in Three Dimensions.}

Cartesian Coordinates. Again, we start with the simplest case, cartesian coordinates $(x, y, z)$ $=\vec{x} \in \mathbb{R}^{3} \equiv E^{(3)}$. Then $\left(g_{a b}\right)=\mathbb{1}_{3}$, and $\vec{p}=-\mathrm{i} \hbar \nabla$. This gives for the Hamiltonian

$$
-\frac{\hbar^{2}}{2 M} \Delta_{E^{(3)}}=-\frac{\hbar^{2}}{2 M} \nabla^{2}=\frac{1}{2 M} \vec{p}^{2},
$$

The most general potential separable in cartesian coordinates has the form

$$
V(\vec{x})=u(x)+v(y)+w(z) .
$$


Circular Polar Coordinates. Next, we consider circular cylinder coordinates which are very similar to the two-dimensional polar coordinates

$$
\left.\begin{array}{ll}
x=\rho \cos \phi, & \rho>0, \\
y=\rho \sin \phi, & 0 \leq \phi<2 \pi, \\
z=z, & z \in \mathbb{R} .
\end{array}\right\}
$$

The metric reads $\left(g_{a b}\right)=\operatorname{diag}\left(1, \rho^{2}, 1\right)$, and the momentum operators are given by (14) and $p_{z}=-\mathrm{i} \hbar \partial_{z}$. This gives for the Hamiltonian

$$
-\frac{\hbar^{2}}{2 M} \Delta_{E^{(3)}}=-\frac{\hbar^{2}}{2 M}\left(\frac{\partial^{2}}{\partial \rho^{2}}+\frac{1}{\rho} \frac{\partial}{\partial \rho}+\frac{1}{\rho^{2}} \frac{\partial^{2}}{\partial \phi^{2}}+\frac{\partial^{2}}{\partial z^{2}}\right)=\frac{1}{2 M}\left(p_{\rho}^{2}+\frac{1}{\rho^{2}} p_{\phi}^{2}+p_{z}^{2}\right)-\frac{\hbar^{2}}{8 M \rho^{2}} .
$$

A separable potential has the form

$$
V(\vec{x})=u(\rho)+\frac{1}{\rho^{2}} v(\phi)+w(z) .
$$

Circular Elliptic coordinates. We consider the coordinate system

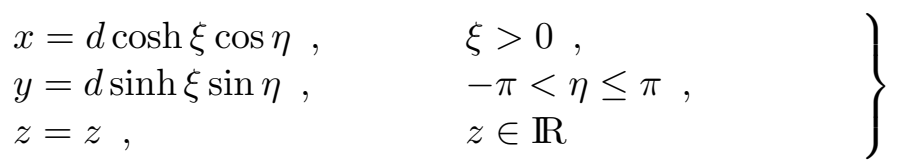

(alternatively $\xi \in \mathbb{R}, 0<\eta<\pi[97])$. The metric is $\left(g_{a b}\right)=\operatorname{diag}\left[d^{2}\left(\sinh ^{2} \xi+\sin ^{2} \eta\right), d^{2}\left(\sinh ^{2} \xi+\right.\right.$ $\left.\left.\sin ^{2} \eta\right), 1\right]$, and we obtain for the momentum operators (18), and $p_{z}=-\mathrm{i} \hbar \partial_{z}$. Consequently for the Hamiltonian

$$
\begin{aligned}
-\frac{\hbar^{2}}{2 M} \Delta_{E^{(3)}} & =-\frac{\hbar^{2}}{2 M}\left[\frac{1}{d^{2}\left(\sinh ^{2} \xi+\sin ^{2} \eta\right)}\left(\frac{\partial^{2}}{\partial \xi^{2}}+\frac{\partial^{2}}{\partial \eta^{2}}\right)+\frac{\partial^{2}}{\partial z^{2}}\right] \\
& =\frac{1}{2 M}\left[\frac{1}{d^{2}}\left(\sinh ^{2} \xi+\sin ^{2} \eta\right)^{-1 / 2}\left(p_{\xi}^{2}+p_{\eta}^{2}\right)\left(\sinh ^{2} \xi+\sin ^{2} \eta\right)^{-1 / 2}+p_{z}^{2}\right] .
\end{aligned}
$$

Similarly as in the two-dimensional case, circular elliptic II coordinates are described by the replacement $x \mapsto x=d(\cosh \xi \cos \eta+1)$. A potential separable in elliptic coordinates reads

$$
V(\vec{x})=\frac{u(\cosh \xi)+v(\cos \eta)}{\sinh ^{2} \xi+\sin ^{2} \eta}+w(z)
$$

Circular Parabolic Coordinates. The last example for cylinder coordinates in three dimensions are the parabolic cylinder coordinates

$$
\begin{aligned}
& x=\frac{1}{2}\left(\eta^{2}-\xi^{2}\right), \quad y=\xi \eta, \quad \xi \in \mathbb{R}, \eta>0, \\
& z=z, \quad z \in \mathbb{R} \text {, }
\end{aligned}
$$

which is the obvious generalization of the two-dimensional case. We have $\left(g_{a b}\right)=\operatorname{diag}\left(\xi^{2}+\right.$ $\eta^{2}, \xi^{2}+\eta^{2}, 1$ ), hence for the momentum operators (22) and $p_{z}=-\mathrm{i} \hbar \partial_{z}$. This gives for the Hamiltonian

$$
\begin{aligned}
-\frac{\hbar^{2}}{2 M} \Delta_{E^{(3)}} & =-\frac{\hbar^{2}}{2 M}\left[\frac{1}{\xi^{2}+\eta^{2}}\left(\frac{\partial^{2}}{\partial \xi^{2}}+\frac{\partial^{2}}{\partial \eta^{2}}\right)+\frac{\partial^{2}}{\partial z^{2}}\right] \\
& =\frac{1}{2 M}\left[\left(\xi^{2}+\eta^{2}\right)^{-1 / 2}\left(p_{\xi}^{2}+p_{\eta}^{2}\right)\left(\xi^{2}+\eta^{2}\right)^{-1 / 2}+p_{z}^{2}\right] .
\end{aligned}
$$

A separable potential has the form

$$
V(\vec{x})=\frac{u(\xi)+v(\eta)}{\xi^{2}+\eta^{2}}+w(z)
$$


Sphero-Conical Coordinates. We consider the coordinate system in terms of Jacobi functions [11]

$$
\left.\begin{array}{ll}
x=r \operatorname{sn}(\mu, k) \operatorname{dn}\left(\nu, k^{\prime}\right), & r>0, \quad k^{2}+k^{\prime 2}=1, \\
y=r \operatorname{cn}(\mu, k) \operatorname{cn}\left(\nu, k^{\prime}\right), & -k \leq \mu \leq k \\
z=r \operatorname{dn}(\mu, k) \operatorname{sn}\left(\nu, k^{\prime}\right), & -2 k^{\prime} \leq \nu \leq 2 k^{\prime} .
\end{array}\right\}
$$

The metric tensor $g_{a b}$ in these coordinates has the form

$$
\left(g_{a b}\right)=\operatorname{diag}\left[1, r^{2}\left(k^{2} \mathrm{cn}^{2} \mu+{k^{\prime}}^{2} \mathrm{cn}^{2} \nu\right), r^{2}\left(k^{2} \mathrm{cn}^{2} \mu+{k^{\prime}}^{2} \mathrm{cn}^{2} \nu\right)\right],
$$

the momentum operators are $p_{r}=-\mathrm{i} \hbar(\partial / \partial r+1 / r)$, and

$$
p_{\mu}=\frac{\hbar}{\mathrm{i}}\left(\frac{\partial}{\partial \mu}-\frac{k^{2} \operatorname{sn} \mu \operatorname{cn} \mu \mathrm{dn} \mu}{k^{2} \operatorname{cn}^{2} \mu+k^{\prime 2} \operatorname{cn}^{2} \nu}\right), \quad p_{\nu}=\frac{\hbar}{\mathrm{i}}\left(\frac{\partial}{\partial \nu}-\frac{k^{\prime 2} \operatorname{sn} \nu \operatorname{cn} \nu \operatorname{dn} \nu}{k^{2} \operatorname{cn}^{2} \mu+k^{\prime 2} \operatorname{cn}^{2} \nu}\right) .
$$

The Hamiltonian has the form

$$
\begin{aligned}
-\frac{\hbar^{2}}{2 M} \Delta_{E^{(3)}} & =-\frac{\hbar^{2}}{2 M}\left[\frac{\partial^{2}}{\partial r^{2}}+\frac{2}{r} \frac{\partial}{\partial r}+\frac{1}{r^{2}} \frac{1}{k^{2} \operatorname{cn}^{2} \mu+k^{\prime 2} \operatorname{cn}^{2} \nu}\left(\frac{\partial^{2}}{\partial \mu^{2}}+\frac{\partial^{2}}{\partial \nu^{2}}\right)\right] \\
& =\frac{1}{2 M}\left(p_{r}^{2}+\frac{1}{r^{2}} \frac{1}{\sqrt{k^{2} \mathrm{cn}^{2} \mu+k^{\prime 2} \operatorname{cn}^{2} \nu}}\left(p_{\mu}^{2}+p_{\nu}^{2}\right) \frac{1}{\sqrt{k^{2} \operatorname{cn}^{2} \mu+k^{\prime 2} \operatorname{cn}^{2} \nu}}\right) .
\end{aligned}
$$

Here a separable potential must have the form

$$
V(\vec{x})=u(r)+\frac{1}{r^{2}} \frac{v(\operatorname{cn} \alpha)+w(\operatorname{cn} \beta)}{k^{2} \operatorname{cn}^{2} \alpha+k^{\prime 2} \operatorname{cn}^{2} \beta} .
$$

The algebraic representation of these coordinates has the form

$$
\begin{aligned}
x^{2} & =r^{2} \frac{\left(\rho_{1}-a_{1}\right)\left(\rho_{2}-a_{1}\right)}{\left(a_{3}-a_{1}\right)\left(a_{2}-a_{1}\right)}, & & r>0, \\
y^{2} & =r^{2} \frac{\left(\rho_{1}-a_{2}\right)\left(\rho_{2}-a_{2}\right)}{\left(a_{3}-a_{2}\right)\left(a_{1}-a_{2}\right)}, & & a_{1}<\rho_{1}<a_{2}<\rho_{2}<a_{3}, \\
z^{2} & =r^{2} \frac{\left(\rho_{1}-a_{3}\right)\left(\rho_{2}-a_{3}\right)}{\left(a_{1}-a_{3}\right)\left(a_{2}-a_{3}\right)} . & &
\end{aligned}
$$

Here we have $\left[P(\rho)=\left(\rho-a_{1}\right)\left(\rho-a_{2}\right)\left(\rho-a_{3}\right)\right]$

$$
\left(g_{a b}\right)=\operatorname{diag}\left(1, \frac{r^{2}}{4} \frac{\rho_{2}-\rho_{1}}{P\left(\rho_{1}\right)}, \frac{r^{2}}{4} \frac{\rho_{1}-\rho_{2}}{P\left(\rho_{2}\right)}\right) .
$$

The momentum operators are

$$
p_{\rho_{1}}=\frac{\hbar}{\mathrm{i}}\left(\frac{\partial}{\partial \rho_{1}}+\frac{1}{2} \frac{1}{\rho_{1}-\rho_{2}}-\frac{1}{4} \frac{P^{\prime}\left(\rho_{1}\right)}{P\left(\rho_{1}\right)}\right), \quad p_{\rho_{2}}=\frac{\hbar}{\mathrm{i}}\left(\frac{\partial}{\partial \rho_{2}}+\frac{1}{2} \frac{1}{\rho_{2}-\rho_{1}}-\frac{1}{4} \frac{P^{\prime}\left(\rho_{2}\right)}{P\left(\rho_{2}\right)}\right) .
$$

Together with the zweibeins

$$
h^{\rho_{1} \rho_{1}}=\frac{1}{2} \sqrt{\frac{P\left(\rho_{1}\right)}{\rho_{2}-\rho_{1}}}, \quad h^{\rho_{2} \rho_{2}}=\frac{1}{2} \sqrt{\frac{P\left(\rho_{2}\right)}{\rho_{1}-\rho_{2}}}
$$


we obtain for the Hamiltonian

$$
\begin{aligned}
H & =-\frac{\hbar^{2}}{2 M}\left(\frac{\partial^{2}}{\partial r^{2}}+\frac{2}{r} \frac{\partial}{\partial r}+\frac{1}{r^{2}} \Delta_{L B}\right) \\
& =\frac{1}{2 M}\left[p_{r}^{2}+\frac{1}{r^{2}}\left(\sum_{i=1}^{2} h^{\rho_{i} \rho_{i}} p_{\rho_{i}}^{2} h^{\rho_{i} \rho_{i}}+\Delta V_{P F}\left(\rho_{i}\right)\right)\right]-\frac{\hbar^{2}}{8 M r^{2}}, \\
\Delta_{L B} & =\frac{4}{\rho_{2}-\rho_{1}}\left[\sqrt{P\left(\rho_{1}\right)} \frac{\partial}{\partial \rho_{1}} \sqrt{P\left(\rho_{1}\right)} \frac{\partial}{\partial \rho_{1}}+\sqrt{-P\left(\rho_{2}\right)} \frac{\partial}{\partial \rho_{2}} \sqrt{-P\left(\rho_{2}\right)} \frac{\partial}{\partial \rho_{2}}\right],
\end{aligned}
$$

where the $\Delta V_{P F}\left(\rho_{i}\right)$ are determined by (10). The relation between the coordinates $\left(\rho_{1}, \rho_{2}\right)$ and $(\mu, \nu)$ is established via

$$
\left(a_{1}-\rho_{1}\right)=\left(a_{1}-a_{2}\right) \operatorname{sn}^{2}(\mu, k), \quad\left(a_{2}-\rho_{2}\right)=\left(a_{2}-a_{3}\right) \mathrm{cn}^{2}\left(\nu, k^{\prime}\right) .
$$

Spherical Coordinates. We consider the spherical coordinates

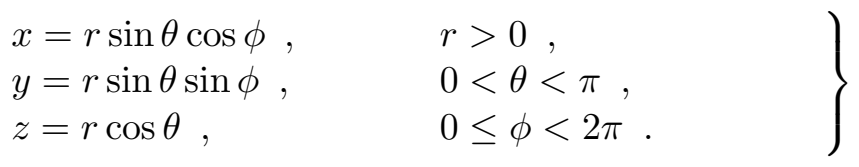

These are the usual three-dimensional polar coordinates. The metric tensor is $\left(g_{a b}\right)=$ $\operatorname{diag}\left(1, r^{2}, r^{2} \sin ^{2} \theta\right)$, and the momentum operators have the form

$$
p_{r}=\frac{\hbar}{\mathrm{i}}\left(\frac{\partial}{\partial r}+\frac{1}{r}\right), \quad p_{\theta}=\frac{\hbar}{\mathrm{i}}\left(\frac{\partial}{\partial \theta}+\frac{1}{2} \cot \theta\right), \quad p_{\phi}=\frac{\hbar}{\mathrm{i}} \frac{\partial}{\partial \phi} .
$$

For the Hamiltonian we obtain

$$
\begin{aligned}
-\frac{\hbar^{2}}{2 M} \Delta_{E^{(3)}} & =-\frac{\hbar^{2}}{2 M}\left[\frac{\partial^{2}}{\partial r^{2}}+\frac{2}{r} \frac{\partial}{\partial r}+\frac{1}{r^{2}}\left(\frac{\partial^{2}}{\partial \theta^{2}}+\cot \theta \frac{\partial}{\partial \theta}\right)+\frac{1}{r^{2} \sin ^{2} \theta} \frac{\partial^{2}}{\partial \phi^{2}}\right] \\
& =\frac{1}{2 M}\left(p_{r}^{2}+\frac{1}{r^{2}} p_{\theta}^{2}+\frac{1}{r^{2} \sin ^{2} \theta} p_{\phi}^{2}\right)-\frac{\hbar^{2}}{8 M r^{2}}\left(1+\frac{1}{\sin ^{2} \theta}\right) .
\end{aligned}
$$

A potential which is separable in spherical coordinates reads

$$
V(\vec{x})=u(r)+\frac{1}{r^{2}} v(\theta)+\frac{1}{r^{2} \sin ^{2} \theta} w(\phi) .
$$

Parabolic Coordinates. We consider the coordinate system

$$
\left.\begin{array}{ll}
x=\xi \eta \cos \phi, & y=\xi \eta \sin \phi,
\end{array} \quad \begin{array}{l}
\xi, \eta>0 \\
z=\frac{1}{2}\left(\xi^{2}-\eta^{2}\right),
\end{array}\right\}
$$

This gives for the metric tensor $\left(g_{a b}\right)=\operatorname{diag}\left(\xi^{2}+\eta^{2}, \xi^{2}+\eta^{2}, \xi^{2} \eta^{2}\right)$, and for the momentum operators we get

$$
p_{\xi}=\frac{\hbar}{\mathrm{i}}\left(\frac{\partial}{\partial \xi}+\frac{\xi}{\xi^{2}+\eta^{2}}+\frac{1}{2 \xi}\right), \quad p_{\eta}=\frac{\hbar}{\mathrm{i}}\left(\frac{\partial}{\partial \eta}+\frac{\eta}{\xi^{2}+\eta^{2}}+\frac{1}{2 \eta}\right),
$$

together with $p_{\phi}=-\mathrm{i} \hbar \partial_{\phi}$. For the Hamiltonian we obtain

$$
\begin{aligned}
-\frac{\hbar^{2}}{2 M} \Delta_{E^{(3)}} & =-\frac{\hbar^{2}}{2 M}\left[\frac{1}{\xi^{2}+\eta^{2}}\left(\frac{\partial^{2}}{\partial \xi^{2}}+\frac{\partial}{\partial \xi}+\frac{\partial^{2}}{\partial \eta^{2}}+\frac{\partial}{\partial \eta}\right)+\frac{1}{\xi^{2} \eta^{2}} \frac{\partial^{2}}{\partial \phi^{2}}\right] \\
& =\frac{1}{2 M}\left[\frac{1}{\sqrt{\xi^{2}+\eta^{2}}}\left(p_{\xi}^{2}+p_{\eta}^{2}\right) \frac{1}{\sqrt{\xi^{2}+\eta^{2}}}+\frac{1}{\xi^{2} \eta^{2}} p_{\phi}^{2}\right]-\frac{\hbar^{2}}{8 M \xi^{2} \eta^{2}} .
\end{aligned}
$$


In parabolic coordinates a potential is separable if it has the form

$$
V(\vec{x})=\frac{u(\xi)+v(\eta)}{\sqrt{x^{2}+y^{2}+z^{2}}}+\frac{w(\phi)}{x^{2}+y^{2}}
$$

Prolate Spheroidal Coordinates. We consider the coordinate system

$$
\begin{array}{ll}
x=\frac{R}{2} \sqrt{\left(\xi^{2}-1\right)\left(1-\eta^{2}\right)} \cos \phi=d \sinh \mu \sin \nu \cos \phi, & \mu>0, \quad 0<\nu<\pi, \\
y=\frac{R}{2} \sqrt{\left(\xi^{2}-1\right)\left(1-\eta^{2}\right)} \sin \phi=d \sinh \mu \sin \nu \sin \phi, & \xi>1, \quad|\eta|<1, \\
z=\frac{R}{2} \xi \eta=d \cosh \mu \cos \nu, & 0 \leq \phi<2 \pi .
\end{array}
$$

For convenience we have also introduced the alternative representation of the coordinates in terms of trigonometric and hyperbolic functions via $\xi=\cosh \mu, \eta=\cos \nu . R=2 d$ is the interfocus distance. This yields $\left(g_{a b}\right)=d^{2} \operatorname{diag}\left(\sinh ^{2} \mu+\sin ^{2} \nu, \sinh ^{2} \mu+\sin ^{2} \nu, \sinh ^{2} \mu \sin ^{2} \nu\right)$, and for the momentum operators we obtain

$$
p_{\mu}=\frac{\hbar}{\mathrm{i}}\left(\frac{\partial}{\partial \mu}+\frac{\sinh \mu \cosh \mu}{\sinh ^{2} \mu+\sin ^{2} \nu}+\frac{1}{2} \operatorname{coth} \mu\right), p_{\nu}=\frac{\hbar}{\mathrm{i}}\left(\frac{\partial}{\partial \nu}+\frac{\sin \nu \cos \nu}{\sinh ^{2} \mu+\sin ^{2} \nu}+\frac{1}{2} \cot \nu\right),
$$

and $p_{\phi}=-\mathrm{i} \hbar \partial_{\phi}$. The Hamiltonian has the form

$$
\begin{gathered}
-\frac{\hbar^{2}}{2 M} \Delta_{E^{(3)}} \\
=-\frac{\hbar^{2}}{2 M d^{2}}\left[\frac{1}{\sinh ^{2} \mu+\sin ^{2} \nu}\left(\frac{\partial^{2}}{\partial \mu^{2}}+\operatorname{coth} \mu \frac{\partial}{\partial \mu}+\frac{\partial^{2}}{\partial \nu^{2}}+\cot \nu \frac{\partial}{\partial \nu}\right)+\frac{1}{\sinh ^{2} \mu \sin ^{2} \nu} \frac{\partial^{2}}{\partial^{2} \phi}\right] \\
=\frac{1}{2 M d^{2}}\left[\frac{1}{\sqrt{\sinh ^{2} \mu+\sin ^{2} \nu}}\left(p_{\mu}^{2}+p_{\nu}^{2}\right) \frac{1}{\sqrt{\sinh ^{2} \mu+\sin ^{2} \nu}}+\frac{1}{\sinh ^{2} \mu+\sin ^{2} \nu} p_{\phi}^{2}\right] \\
-\frac{\hbar^{2}}{8 M d^{2} \sinh ^{2} \mu \sin ^{2} \nu} .
\end{gathered}
$$

A separable potential must have the form

$$
V(\vec{x})=\frac{u(\cosh \mu)+v(\cos \nu)}{\sinh ^{2} \mu+\sin ^{2} \nu}+\frac{w(\phi)}{\sinh ^{2} \mu \sin ^{2} \nu} .
$$

Replacing $z \mapsto z=d(\cosh \mu \cos \nu+1)$ gives the prolate spheroidal II coordinate system [22, 75, 83, 94, 128], which is one of the four coordinate systems which separate the Coulomb potential problem, c.f. the potential $V_{3}(\vec{x})$ in 3.2.3. Note that there is no mathematical difference between the prolate spheroidal and prolate spheroidal II coordinate systems. The later has a shifted origin such that the left focus is located at the coordinate origin. As in the elliptic coordinate system in two dimensions the prolate (and oblate) spheroidal coordinate system is a one parameter coordinate system. The prolate spheroidal coordinate has the property of separating the twocenter Coulomb problem (Coulson and Robinson [22], Morse [104] and Teller [124]).

Oblate Spheroidal Coordinates. We consider the coordinate system

$$
\begin{aligned}
& x=\frac{\bar{R}}{2} \sqrt{\left(\bar{\xi}^{2}+1\right)\left(1-\bar{\eta}^{2}\right)} \cos \phi=\bar{d} \cosh \bar{\mu} \sin \bar{\nu} \cos \phi, \quad \bar{\mu}>0, \quad 0<\bar{\nu}<\pi, \\
& y=\frac{\bar{R}}{2} \sqrt{\left(\bar{\xi}^{2}+1\right)\left(1-\bar{\eta}^{2}\right)} \sin \phi=\bar{d} \cosh \bar{\mu} \sin \bar{\nu} \sin \phi, \quad \bar{\xi}>0, \quad|\bar{\eta}|<1, \\
& z=\frac{\bar{R}}{2} \bar{\xi} \bar{\eta}=\bar{d} \sinh \bar{\mu} \cos \bar{\nu}, \quad 0 \leq \phi<2 \pi
\end{aligned}
$$


(alternatively $\bar{\mu} \in \mathbb{R}, 0<\bar{\nu}<\pi / 2$ [97]). We again use also the alternative representation $\bar{\xi}=\sinh \bar{\mu}, \bar{\eta}=\cos \bar{\nu} . \quad \bar{R}=2 \bar{d}$ is the interfocus distance. This yields $\left(g_{a b}\right)=\bar{d}^{2} \operatorname{diag}\left(\cosh ^{2} \bar{\mu}-\right.$ $\left.\sin ^{2} \bar{\nu}, \cosh ^{2} \bar{\mu}-\sin ^{2} \bar{\nu}, \cosh ^{2} \bar{\mu} \sin ^{2} \bar{\nu}\right)$, and for the momentum operators we obtain

$$
p_{\bar{\mu}}=\frac{\hbar}{\mathrm{i}}\left(\frac{\partial}{\partial \bar{\mu}}+\frac{\sinh \bar{\mu} \cosh \bar{\mu}}{\cosh ^{2} \bar{\mu}-\sin ^{2} \bar{\nu}}+\frac{1}{2} \tanh \bar{\mu}\right), p_{\bar{\nu}}=\frac{\hbar}{\mathrm{i}}\left(\frac{\partial}{\partial \bar{\nu}}+\frac{\sin \bar{\nu} \cos \bar{\nu}}{\cosh ^{2} \bar{\mu}-\sin ^{2} \bar{\nu}}+\frac{1}{2} \cot \bar{\nu}\right),
$$

and $p_{\phi}=-\mathrm{i} \hbar \partial_{\phi}$. The Hamiltonian has the form

$$
\begin{gathered}
-\frac{\hbar^{2}}{2 M} \Delta_{E^{(3)}} \\
=-\frac{\hbar^{2}}{2 M \bar{d}^{2}}\left[\frac{1}{\cosh ^{2} \bar{\mu}-\sin ^{2} \bar{\nu}}\left(\frac{\partial^{2}}{\partial \bar{\mu}^{2}}+\tanh \bar{\mu} \frac{\partial}{\partial \bar{\mu}}+\frac{\partial^{2}}{\partial \bar{\nu}^{2}}+\cot \bar{\nu} \frac{\partial}{\partial \bar{\nu}}\right)+\frac{1}{\cosh ^{2} \bar{\mu} \sin ^{2} \bar{\nu}} \frac{\partial^{2}}{\partial^{2} \phi}\right] \\
=\frac{1}{2 M \bar{d}^{2}}\left[\frac{1}{\sqrt{\cosh ^{2} \bar{\mu}-\sin ^{2} \bar{\nu}}}\left(p_{\bar{\mu}}^{2}+p_{\bar{\nu}}^{2}\right) \frac{1}{\sqrt{\cosh ^{2} \bar{\mu}-\sin ^{2} \bar{\nu}}}+\frac{1}{\cosh ^{2} \bar{\mu}-\sin ^{2} \bar{\nu}} p_{\phi}^{2}\right] \\
-\frac{\hbar^{2}}{8 M \bar{d}^{2} \cosh ^{2} \bar{\mu} \sin ^{2} \bar{\nu}} .
\end{gathered}
$$

Here a separable potential must have the form

$$
V(\vec{x})=\frac{u(\sinh \bar{\mu})+v(\cos \bar{\nu})}{\cosh ^{2} \bar{\mu}-\sin ^{2} \bar{\nu}}+\frac{w(\phi)}{\cosh ^{2} \bar{\mu} \sin ^{2} \bar{\nu}} .
$$

Paraboloidal Coordinates. The last two coordinate system are the most complicated ones and are similar in some of their features, c.f. (80, 81) and [58, 105, 106] for further information. First we consider the coordinate system

$$
\begin{aligned}
& x^{2}=\frac{\left(\eta_{1}-a\right)\left(\eta_{2}-a\right)\left(\eta_{3}-a\right)}{a-b}, 0<\eta_{1}<b<\eta_{2}<a<\eta_{3} \\
& y^{2}=\frac{\left(\eta_{1}-b\right)\left(\eta_{2}-b\right)\left(\eta_{3}-b\right)}{b-a}, \\
& z=\frac{1}{2}\left(\eta_{1}+\eta_{2}+\eta_{3}-a-b\right),
\end{aligned}
$$

The metric tensor is given by $[P(\eta)=(\eta-a)(\eta-b)]$

$$
\left(g_{a b}\right)=\frac{1}{4} \operatorname{diag}\left(\frac{\left(\eta_{1}-\eta_{2}\right)\left(\eta_{1}-\eta_{3}\right)}{P\left(\eta_{1}\right)}, \frac{\left(\eta_{2}-\eta_{1}\right)\left(\eta_{2}-\eta_{3}\right)}{P\left(\eta_{2}\right)}, \frac{\left(\eta_{3}-\eta_{1}\right)\left(\eta_{3}-\eta_{2}\right)}{P\left(\eta_{3}\right)}\right) .
$$

The momentum operators are

$$
\begin{aligned}
& p_{\eta_{1}}=\frac{\hbar}{\mathrm{i}}\left(\frac{\partial}{\partial \eta_{1}}+\frac{1}{2} \frac{1}{\eta_{1}-\eta_{2}}+\frac{1}{2} \frac{1}{\eta_{1}-\eta_{3}}-\frac{1}{4} \frac{P^{\prime}\left(\eta_{1}\right)}{P\left(\eta_{1}\right)}\right), \\
& p_{\eta_{2}}=\frac{\hbar}{\mathrm{i}}\left(\frac{\partial}{\partial \eta_{2}}+\frac{1}{2} \frac{1}{\eta_{2}-\eta_{1}}+\frac{1}{2} \frac{1}{\eta_{2}-\eta_{3}}-\frac{1}{4} \frac{P^{\prime}\left(\eta_{2}\right)}{P\left(\eta_{2}\right)}\right), \\
& p_{\eta_{3}}=\frac{\hbar}{\mathrm{i}}\left(\frac{\partial}{\partial \eta_{3}}+\frac{1}{2} \frac{1}{\eta_{3}-\eta_{1}}+\frac{1}{2} \frac{1}{\eta_{3}-\eta_{2}}-\frac{1}{4} \frac{P^{\prime}\left(\eta_{3}\right)}{P\left(\eta_{3}\right)}\right) .
\end{aligned}
$$

Together with the dreibeins

$$
\left.\begin{array}{rl}
h^{\eta_{1} \eta_{1}} & =\frac{1}{2} \sqrt{\frac{P\left(\eta_{1}\right)}{\left(\eta_{1}-\eta_{2}\right)\left(\eta_{1}-\eta_{3}\right.}}, \\
h^{\eta_{2} \eta_{2}} & =\frac{1}{2} \sqrt{\frac{P\left(\eta_{2}\right)}{\left(\eta_{2}-\eta_{1}\right)\left(\eta_{2}-\eta_{3}\right)}}, \\
h^{\eta_{3} \eta_{3}} & =\frac{1}{2} \sqrt{\frac{P\left(\eta_{3}\right)}{\left(\eta_{3}-\eta_{1}\right)\left(\eta_{3}-\eta_{2}\right)}},
\end{array}\right\}
$$


we obtain for the Hamiltonian

$$
\begin{aligned}
-\frac{\hbar^{2}}{2 M} \Delta_{E^{(3)}}= & -\frac{2 \hbar^{2}}{M}\left[\frac{\sqrt{P\left(\eta_{1}\right)}}{\left(\eta_{1}-\eta_{2}\right)\left(\eta_{1}-\eta_{3}\right)} \frac{\partial}{\partial \eta_{1}} \sqrt{P\left(\eta_{1}\right)} \frac{\partial}{\partial \eta_{1}}+\frac{\sqrt{P\left(\eta_{2}\right)}}{\left(\eta_{2}-\eta_{3}\right)\left(\eta_{2}-\eta_{1}\right)} \frac{\partial}{\partial \eta_{2}} \sqrt{P\left(\eta_{2}\right)} \frac{\partial}{\partial \eta_{2}}\right. \\
& \left.+\frac{\sqrt{P\left(\eta_{3}\right)}}{\left(\eta_{3}-\eta_{1}\right)\left(\eta_{3}-\eta_{2}\right)} \frac{\partial}{\partial \eta_{3}} \sqrt{P\left(\eta_{3}\right)} \frac{\partial}{\partial \eta_{3}}\right] \\
= & \frac{1}{2 M} \sum_{i=1}^{3}\left(h^{\eta_{i} \eta_{i}} p_{\eta_{i}}^{2} h^{\eta_{i} \eta_{i}}+\Delta V\left(\eta_{i}\right)\right)
\end{aligned}
$$

The $\Delta V_{P F}\left(\eta_{i}\right)$ are determined by (10). A potential separable in paraboloidal coordinates must have the form

$$
V(\vec{x})=\frac{\left(\eta_{2}-\eta_{3}\right) u\left(\eta_{1}\right)+\left(\eta_{1}-\eta_{3}\right) v\left(\eta_{2}\right)+\left(\eta_{1}-\eta_{2}\right) w\left(\eta_{3}\right)}{\left(\eta_{1}-\eta_{2}\right)\left(\eta_{1}-\eta_{3}\right)\left(\eta_{2}-\eta_{3}\right)}
$$

Ellipsoidal Coordinates. As the last coordinate system we consider

$$
\begin{aligned}
x^{2} & =\frac{\left(\rho_{1}-a_{3}\right)\left(\rho_{2}-a_{3}\right)\left(\rho_{3}-a_{3}\right)}{\left(a_{3}-a_{1}\right)\left(a_{2}-a_{1}\right)}, 0<a_{1}<\rho_{1}<a_{2}<\rho_{2}<a_{3}<\rho_{3}, \\
y^{2} & =\frac{\left(\rho_{1}-a_{2}\right)\left(\rho_{2}-a_{2}\right)\left(\rho_{3}-a_{2}\right)}{\left(a_{3}-a_{2}\right)\left(a_{1}-a_{2}\right)}, \\
z^{2} & =\frac{\left(\rho_{1}-a_{1}\right)\left(\rho_{2}-a_{1}\right)\left(\rho_{3}-a_{1}\right)}{\left(a_{1}-a_{3}\right)\left(a_{2}-a_{3}\right)} .
\end{aligned}
$$

For the metric tensor we have $\left[P(\rho)=\left(\rho-a_{1}\right)\left(\rho-a_{2}\right)\left(\rho-a_{3}\right)\right]$

$$
\left(g_{a b}\right)=\frac{1}{4} \operatorname{diag}\left(\frac{\left(\rho_{1}-\rho_{2}\right)\left(\rho_{1}-\rho_{3}\right)}{P\left(\rho_{1}\right)}, \frac{\left(\rho_{2}-\rho_{3}\right)\left(\rho_{2}-\rho_{1}\right)}{P\left(\rho_{2}\right)}, \frac{\left(\rho_{3}-\rho_{1}\right)\left(\rho_{3}-\rho_{2}\right)}{P\left(\rho_{3}\right)}\right) .
$$

The momentum operators are

$$
\begin{aligned}
& p_{\rho_{1}}=\frac{\hbar}{\mathrm{i}}\left(\frac{\partial}{\partial \rho_{1}}+\frac{1}{2} \frac{1}{\rho_{1}-\rho_{2}}+\frac{1}{2} \frac{1}{\rho_{1}-\rho_{3}}-\frac{1}{4} \frac{P^{\prime}\left(\rho_{1}\right)}{P\left(\rho_{1}\right)}\right), \\
& p_{\rho_{2}}=\frac{\hbar}{\mathrm{i}}\left(\frac{\partial}{\partial \rho_{2}}+\frac{1}{2} \frac{1}{\rho_{2}-\rho_{1}}+\frac{1}{2} \frac{1}{\rho_{2}-\rho_{3}}-\frac{1}{4} \frac{P^{\prime}\left(\rho_{2}\right)}{P\left(\rho_{2}\right)}\right), \\
& p_{\rho_{3}}=\frac{\hbar}{\mathrm{i}}\left(\frac{\partial}{\partial \rho_{3}}+\frac{1}{2} \frac{1}{\rho_{3}-\rho_{1}}+\frac{1}{2} \frac{1}{\rho_{3}-\rho_{2}}-\frac{1}{4} \frac{P^{\prime}\left(\rho_{3}\right)}{P\left(\rho_{3}\right)}\right) .
\end{aligned}
$$

Together with the dreibeins

$$
\left.\begin{array}{rl}
h^{\rho_{1} \rho_{1}} & =\frac{1}{2} \sqrt{\frac{P\left(\rho_{1}\right)}{\left(\rho_{1}-\rho_{2}\right)\left(\rho_{1}-\rho_{3}\right.}}, \\
h^{\rho_{2} \rho_{2}} & =\frac{1}{2} \sqrt{\frac{P\left(\rho_{2}\right)}{\left(\rho_{2}-\rho_{1}\right)\left(\rho_{2}-\rho_{3}\right)}}, \\
h^{\rho_{3} \rho_{3}} & =\frac{1}{2} \sqrt{\frac{P\left(\rho_{3}\right)}{\left(\rho_{3}-\rho_{1}\right)\left(\rho_{3}-\rho_{2}\right)}},
\end{array}\right\}
$$

we obtain for the Hamiltonian

$$
\begin{aligned}
-\frac{\hbar^{2}}{2 M} \Delta_{E^{(3)}}= & -\frac{2 \hbar^{2}}{M}\left[\frac{\sqrt{P\left(\rho_{1}\right)}}{\left(\rho_{1}-\rho_{2}\right)\left(\rho_{1}-\rho_{3}\right)} \frac{\partial}{\partial \rho_{1}} \sqrt{P\left(\rho_{1}\right)} \frac{\partial}{\partial \rho_{1}}+\frac{\sqrt{P\left(\rho_{2}\right)}}{\left(\rho_{2}-\rho_{3}\right)\left(\rho_{2}-\rho_{1}\right)} \frac{\partial}{\partial \rho_{2}} \sqrt{P\left(\rho_{2}\right)} \frac{\partial}{\partial \rho_{2}}\right. \\
& \left.+\frac{\sqrt{P\left(\rho_{3}\right)}}{\left(\rho_{3}-\rho_{1}\right)\left(\rho_{3}-\rho_{2}\right)} \frac{\partial}{\partial \rho_{3}} \sqrt{P\left(\rho_{3}\right)} \frac{\partial}{\partial \rho_{3}}\right] \\
= & \frac{1}{2 M} \sum_{i=1}^{3}\left(h^{\rho_{i} \rho_{i}} p_{\rho_{i}}^{2} h^{\rho_{i} \rho_{i}}+\Delta V\left(\rho_{i}\right)\right) .
\end{aligned}
$$


The $\Delta V_{P F}\left(\rho_{i}\right)$ are determined by (10). A potential separable in ellipsoidal coordinates has the form of (70) with $\eta_{i}$ replaced by $\rho_{i}$.

The ellipsoidal coordinate system is a two parameter coordinate system. Denoting $R_{1}^{2}=$ $a_{2}-a_{1}, R_{2}^{2}=a_{3}-a_{2}$ and $R_{3}^{2}=a_{3}-a_{1}$, the $R_{i}$ must fulfill the constraint $R_{1}^{2}+R_{2}^{2}=R_{3}^{2}$. The $R_{i}$ describe the foci distance from the coordinate origin. By considering the limits $R_{1,2} \rightarrow 0$, respectively $R_{1,2} \rightarrow \infty$ we can generate the seven coordinate systems described in table 1 (note $\left.\bar{\rho}_{i}=\rho_{i}-a_{2}\right)$.

Table 1: The degenerations of the ellipsoidal coordinate system

\begin{tabular}{|c|c|c|c|}
\hline $\begin{array}{l}\text { prolate } \\
\text { spheroidal } \\
\text { system }\end{array}$ & $\begin{array}{l}x=(R / 2) \sqrt{\left(\xi^{2}-1\right)\left(1-\eta^{2}\right)} \cos \phi \\
y=(R / 2) \sqrt{\left(\xi^{2}-1\right)\left(1-\eta^{2}\right)} \sin \phi \\
z=(R / 2) \xi \eta\end{array}$ & $\begin{array}{l}R_{2}^{2} \rightarrow 0 \\
R_{1}^{2} \rightarrow R^{2} / 4\end{array}$ & $\begin{aligned} & \bar{\rho}_{1} \rightarrow R_{1}^{2}\left(\eta^{2}-1\right) \\
& \bar{\rho}_{2} \rightarrow R_{2}^{2} \sin ^{2} \phi \\
& \bar{\rho}_{3} \rightarrow R_{1}^{2}\left(\xi^{2}-1\right) \\
&\end{aligned}$ \\
\hline $\begin{array}{l}\text { oblate } \\
\text { spheroidal } \\
\text { system }\end{array}$ & $\begin{array}{l}x=(\bar{R} / 2) \sqrt{\left(\bar{\xi}^{2}-1\right)\left(1-\bar{\eta}^{2}\right)} \cos \phi \\
y=(\bar{R} / 2) \sqrt{\left(\bar{\xi}^{2}-1\right)\left(1-\bar{\eta}^{2}\right)} \sin \phi \\
z=(\bar{R} / 2) \bar{\xi} \bar{\eta}\end{array}$ & $\begin{array}{l}R_{2}^{2} \rightarrow 0 \\
R_{1}^{2} \rightarrow \bar{R}^{2} / 4\end{array}$ & $\begin{array}{l}\bar{\rho}_{1} \rightarrow-R_{1}^{2} \sin ^{2} \phi \\
\bar{\rho}_{2} \rightarrow\left(\bar{R}^{2} / 4\right)\left(1-\bar{\eta}^{2}\right) \\
\bar{\rho}_{3} \rightarrow\left(\bar{R}^{2} / 4\right)\left(1+\bar{\eta}^{2}\right)\end{array}$ \\
\hline $\begin{array}{l}\text { Spherical } \\
\text { system }\end{array}$ & $\begin{array}{l}x=r \sin \theta \cos \phi \\
y=r \sin \theta \sin \phi \\
z=r \cos \theta\end{array}$ & $\begin{array}{l}R_{1}^{2} \rightarrow 0 \\
R_{2}^{2} \rightarrow 0 \\
R_{2}^{2} / R_{1}^{2} \rightarrow 0\end{array}$ & $\begin{array}{l}\bar{\rho}_{1} \rightarrow-R_{1}^{2} \sin ^{2} \theta \\
\bar{\rho}_{2} \rightarrow R_{2}^{2} \sin ^{2} \theta \sin ^{2} \phi \\
\bar{\rho}_{3} \rightarrow r^{2}\end{array}$ \\
\hline $\begin{array}{l}\text { Sphero-conical } \\
\text { system }\end{array}$ & $\begin{array}{l}x=r \operatorname{sn}(\mu, k) \operatorname{dn}\left(\nu, k^{\prime}\right) \\
y=r \operatorname{cn}(\mu, k) \operatorname{cn}\left(\nu, k^{\prime}\right) \\
z=r \operatorname{dn}(\mu, k) \operatorname{sn}\left(\nu, k^{\prime}\right)\end{array}$ & $\begin{array}{l}R_{1}^{2} \rightarrow 0 \\
R_{2}^{2} \rightarrow 0 \\
R_{2}^{2} / R_{1}^{2} \rightarrow k^{2}\end{array}$ & $\begin{array}{l}\bar{\rho}_{1} \rightarrow-k^{\prime 2} R_{3}^{2} \mathrm{cn}^{2}\left(\nu, k^{\prime}\right) \\
\bar{\rho}_{2} \rightarrow k^{2} R_{3}^{2} \mathrm{cn}^{2}(\mu, k) \\
\bar{\rho}_{3} \rightarrow r^{2}\end{array}$ \\
\hline $\begin{array}{l}\text { Circular } \\
\text { elliptic } \\
\text { system }\end{array}$ & $\begin{array}{l}x=(R / 2) \sinh \mu \sin \nu \\
y=(R / 2) \cosh \mu \cos \nu \\
z=z^{\prime}\end{array}$ & $\begin{array}{l}R_{1}^{2} \rightarrow \infty \\
R_{2}^{2}=R^{2} / 4\end{array}$ & $\begin{array}{l}\bar{\rho}_{1} \rightarrow z^{\prime 2}-R_{1}^{2} \\
\bar{\rho}_{2} \rightarrow\left(R^{2} / 4\right) \cos ^{2} \nu \\
\bar{\rho}_{3} \rightarrow\left(R^{2} / 4\right) \cosh ^{2} \mu\end{array}$ \\
\hline $\begin{array}{l}\text { Circular } \\
\text { polar } \\
\text { system }\end{array}$ & $\begin{array}{l}x=\rho \cos \phi \\
y=\rho \sin \phi \\
z=z^{\prime}\end{array}$ & $\begin{array}{l}R_{1}^{2} \rightarrow \infty \\
R_{2}^{2} \rightarrow 0\end{array}$ & $\begin{array}{l}\bar{\rho}_{1} \rightarrow z^{\prime 2}-R_{1}^{2} \\
\bar{\rho}_{2} \rightarrow R^{2} \sin ^{2} \phi \\
\bar{\rho}_{3} \rightarrow \rho^{2}\end{array}$ \\
\hline $\begin{array}{l}\text { Cartesian } \\
\text { system }\end{array}$ & $\begin{array}{l}x=x^{\prime} \\
y=y^{\prime} \\
z=z^{\prime}\end{array}$ & $\begin{array}{l}R_{1}^{2} \rightarrow \infty \\
R_{2}^{2} \rightarrow \infty\end{array}$ & $\begin{array}{l}\bar{\rho}_{1} \rightarrow{z^{\prime}}^{2}-R_{1}^{2} \\
\bar{\rho}_{2} \rightarrow x^{\prime 2}+R_{1}^{2} \\
\bar{\rho}_{3} \rightarrow y^{\prime 2}\end{array}$ \\
\hline
\end{tabular}

We must obtain the remaining three coordinate systems which are not listed in table 1 . The paraboloidal system is obtained by the focus translation $z^{\prime} \rightarrow z-R_{1}$ from the ellipsoidal coordinate system by $R_{1,2}^{2} \rightarrow \infty$ together with $R_{2}^{2} / R_{1} \rightarrow a-b$ which gives three more coordinate systems: The parabolic system then follows from the paraboloidal system in the limit $a \rightarrow b$ (the parabolic system can also be obtained from the prolate spheroidal II system). The circular parabolic system is obtained from the degeneration of the elliptic II system, c.f. 2.1.2.

\subsection{Separation of Coordinates in the Path Integral.}

We want to look for the coordinate systems which separate the relevant partial differential equations, i.e. the Hamiltonian, and, more important from our point of view, the path integral. In order to develop such a theory we consider according to 105 the Lagrangian $\mathcal{L}=\frac{M}{2} \sum_{i=1}^{D} h_{i}^{2} \dot{x}_{i}^{2}$ and the Laplacian $\Delta_{L}$, respectively, in the following way (where only orthogonal coordinate systems are taken into account)

$$
\Delta_{L B}=\sum_{i=1}^{D} \frac{1}{\prod_{j=1}^{D} h_{j}(\{\xi\})} \frac{\partial}{\partial \xi_{i}}\left(\frac{\prod_{k=1}^{D} h_{k}(\{\xi\})}{h_{i}^{2}(\{\xi\})} \frac{\partial}{\partial \xi_{i}}\right)
$$




$$
=: \sum_{i=1}^{D} \frac{1}{\prod_{j=1}^{D} h_{j}(\{\xi\})} \frac{\partial}{\partial \xi_{i}}\left(g_{i}\left(\xi_{1}, \ldots, \xi_{i-1}, \xi_{i+1}, \ldots, \xi_{D}\right) f\left(\xi_{i}\right) \frac{\partial}{\partial \xi_{i}}\right),
$$

where $\{\xi\}$ denotes the set of variables $\left(\xi_{1}, \ldots, \xi_{D}\right)$, and the existence of the functions $f_{i}, g_{i}$ is necessary for the separation [74, 105]. We introduce the Stäckel-determinant [74, 101, 105]

$$
S(\{\xi\})=\operatorname{det}\left(\Phi_{i j}\right)=\prod_{i=1}^{D} \frac{h_{i}(\{\xi\})}{f_{i}\left(\xi_{i}\right)}, M_{i}\left(\xi_{1}, \ldots, \xi_{i-1}, \xi_{i+1}, \ldots, \xi_{D}\right)=\frac{\partial S}{\partial \Phi_{i 1}}=\frac{S(\{\xi\})}{h_{i}^{2}(\{\xi\})},
$$

and abbreviate $\Gamma_{i}=f_{i}^{\prime} / f_{i}$. Then

$$
g_{i}\left(\xi_{1}, \ldots, \xi_{i-1}, \xi_{i+1}, \ldots, \xi_{D}\right)=M_{i}\left(\xi_{1}, \ldots, \xi_{i-1}, \xi_{i+1}, \ldots, \xi_{D}\right) \prod_{\substack{j=1 \\ i \neq j}}^{D} f_{j}\left(\xi_{j}\right)
$$

which fixes the functions $g_{i}$. Introducing the (new) momentum operators $P_{\xi_{i}}=\frac{\hbar}{\mathrm{i}}\left(\partial \xi_{i}+\frac{1}{2} f_{i}^{\prime} / f_{i}\right)$ we obtain according to the general theory the following identity in the path integral [58] by means of the space-time transformation technique (Duru [28], Fischer et al. [40], Refs. [59, 61], Kleinert [85], and Pak and Sökmen [110])

$$
\begin{aligned}
& \prod_{i=1}^{D} \int_{\xi_{i}\left(t^{\prime}\right)=\xi_{i}^{\prime}}^{\xi_{i}\left(t^{\prime \prime}\right)=\xi_{i}^{\prime \prime}} h_{i} \mathcal{D} \xi_{i}(t) \exp \left\{\frac{\mathrm{i}}{\hbar} \int_{t^{\prime}}^{t^{\prime \prime}}\left[\frac{M}{2} \sum_{i=1}^{D} h_{i}^{2} \dot{\xi}_{i}^{2}-\Delta V_{P F}(\{\xi\})\right] d t\right\} \\
& =\prod_{i=1}^{D} \int_{\xi_{i}\left(t^{\prime}\right)=\xi_{i}^{\prime}}^{\xi_{i}\left(t^{\prime \prime}\right)=\xi_{i}^{\prime \prime}} \sqrt{\frac{S}{M_{i}}} \mathcal{D} \xi_{i}(t) \exp \left\{\frac{\mathrm{i}}{\hbar} \int_{t^{\prime}}^{t^{\prime \prime}}\left[\frac{M}{2} S \sum_{i=1}^{D} \frac{\dot{\xi}_{i}^{2}}{M_{i}}-\Delta V_{P F}(\{\xi\})\right] d t\right\} \\
& =\left(S^{\prime} S^{\prime \prime}\right)^{\frac{1}{2}(1-D / 2)} \int_{\mathbb{R}} \frac{d E}{2 \pi \hbar} \mathrm{e}^{-\mathrm{i} E T / \hbar} \int_{0}^{\infty} d s^{\prime \prime} \prod_{i=1}^{D} \int_{\xi_{i}(0)=\xi_{i}^{\prime}}^{\xi_{i}\left(s^{\prime \prime}\right)=\xi_{i}^{\prime \prime}} M_{i}^{-1 / 2} \mathcal{D} \xi_{i}(s) \\
& \quad \times \exp \left\{\frac{\mathrm{i}}{\hbar} \int_{0}^{s^{\prime \prime}}\left[\frac{M}{2} \sum_{i=1}^{D} \frac{\dot{\xi}_{i}^{2}}{M_{i}}+E S-\frac{\hbar^{2}}{8 M} \sum_{i=1}^{D} M_{i}\left(\Gamma_{i}^{2}+2 \Gamma_{i}^{\prime}\right)\right] d s\right\} .
\end{aligned}
$$

Here we have adopted the following lattice formulation of the path integral (c.f. DeWitt [25], Feynman [37], Gervais and Jevicki [47], Refs. [59, 61], McLaughlin and Schulman [92], Mizrahi [98], and Omote [107] for alternative lattice definitions)

$$
\begin{aligned}
& K\left(\vec{q}^{\prime \prime}, \vec{q}^{\prime} ; T\right)= \int_{\vec{q}\left(t^{\prime}\right)=\vec{q}^{\prime}}^{\vec{q}\left(t^{\prime \prime}\right)=\vec{q}^{\prime \prime}} \sqrt{g} \mathcal{D}_{P F} \vec{q}(t) \exp \left\{\frac{\mathrm{i}}{\hbar} \int_{t^{\prime}}^{t^{\prime \prime}}\left[\frac{M}{2} h_{a c}(\vec{q}) h_{c b}(\vec{q}) \dot{q}^{a} \dot{q}^{b}-V(\vec{q})-\Delta V_{P F}(\vec{q})\right] d t\right\} \\
& \equiv \lim _{N \rightarrow \infty}\left(\frac{M}{2 \pi \mathrm{i} \epsilon \hbar}\right)^{N D / 2} \prod_{j=1}^{N-1} \int d \vec{q}_{j} \sqrt{g\left(\vec{q}_{j}\right)} \\
& \quad \times \exp \left\{\frac{\mathrm{i}}{\hbar} \sum_{j=1}^{N}\left[\frac{M}{2 \epsilon} h_{b c}\left(\vec{q}_{j}\right) h_{a c}\left(\vec{q}_{j-1}\right) \Delta q_{j}^{a} \Delta q_{j}^{b}-\epsilon V\left(\vec{q}_{j}\right)-\epsilon \Delta V_{P F}\left(\vec{q}_{j}\right)\right]\right\} \cdot(81)
\end{aligned}
$$

This path integral formulation will be used in the sequel. 


\section{Path Integral Formulation of Smorodinsky-Winternitz Potentials.}

\subsection{Two-Dimensional Smorodinsky-Winternitz Potentials.}

In this subsection we discuss the four two-dimensional Smorodinsky-Winternitz potentials [42]. They are characterized by having three functionally independent integrals of motion, i.e. there are two more operators related to these integrals of motion which commute with the Hamiltonian.

We will state if ever possible both the propagator and its spectral expansion into the wavefunctions and energy-levels. $\vec{x}$ denotes a two-dimensional variable: $\vec{x}=(x, y) \equiv\left(x_{1}, x_{2}\right)$, and without loss of generality it will be assumed that $\vec{x} \in \mathbb{R}^{2}, \mathbb{R}^{3}$.

In the table 2 we list the two-dimensional maximally super-integrable Smorodinsky-Winternitz potentials together with the separating coordinate systems [42]. The cases where an explicit path integration is possible are underlined.

Table 2: The two-dimensional maximally super-integrable potentials

\begin{tabular}{|l|l|}
\hline Potential $V(x, y)$ & $\begin{array}{l}\text { Coordinate } \\
\text { System }\end{array}$ \\
\hline$V_{1}=\frac{M}{2} \omega^{2}\left(x^{2}+y^{2}\right)+\frac{\hbar^{2}}{2 M}\left(\frac{k_{1}^{2}-\frac{1}{4}}{x^{2}}+\frac{k_{2}^{2}-\frac{1}{4}}{y^{2}}\right)$ & Cartesian \\
\hline$V_{2}=\frac{M}{2} \omega^{2}\left(4 x^{2}+y^{2}\right)+k_{1} x+\frac{\hbar^{2}}{2 M} \frac{k_{2}^{2}-\frac{1}{4}}{y^{2}}$ & Ellar \\
\hline$V_{3}=-\frac{\alpha}{\sqrt{x^{2}+y^{2}}}+\frac{\hbar^{2}}{4 M} \frac{1}{\sqrt{x^{2}+y^{2}}}\left(\frac{k_{1}^{2}-\frac{1}{4}}{\sqrt{x^{2}+y^{2}}+x}+\frac{k_{2}^{2}-\frac{1}{4}}{\sqrt{x^{2}+y^{2}}-x}\right)$ & $\underline{\text { Cartesian }}$ \\
\hline$V_{4}=-\frac{\alpha}{\rho}+\sqrt{\frac{2}{\rho}}\left(\beta_{1} \cos \frac{\phi}{2}+\beta_{2} \sin \frac{\phi}{2}\right)$ & $\begin{array}{l}\text { Porabolic } \\
\text { Elliptic II } \\
\text { Parabolic }\end{array}$ \\
\hline & $\underline{\text { Mutually }}$ \\
\hline
\end{tabular}

3.1.1. We consider the potential $\left(k_{1,2}>0\right)$

$$
V_{1}(\vec{x})=\frac{M}{2} \omega^{2}\left(x_{1}^{2}+x_{2}^{2}\right)+\frac{\hbar^{2}}{2 M}\left(\frac{k_{1}^{2}-\frac{1}{4}}{x_{1}^{2}}+\frac{k_{2}^{2}-\frac{1}{4}}{x_{2}^{2}}\right) .
$$

This potential is separable in three coordinate systems. We obtain the path integral formulations, c.f. (81) for their lattice definitions

$$
K^{\left(V_{1}\right)}\left(\vec{x}^{\prime \prime}, \vec{x}^{\prime} ; T\right)
$$

Cartesian Coordinates:

$$
=\int_{\vec{x}\left(t^{\prime}\right)=\vec{x}^{\prime}}^{\vec{x}\left(t^{\prime \prime}\right)=\vec{x}^{\prime \prime}} \mathcal{D} \vec{x}(t) \exp \left\{\frac{\mathrm{i}}{\hbar} \int_{t^{\prime}}^{t^{\prime \prime}}\left[\frac{M}{2}\left(\dot{\vec{x}}^{2}-\omega^{2} \vec{x}^{2}\right)-\frac{\hbar^{2}}{2 M}\left(\frac{k_{1}^{2}-\frac{1}{4}}{x_{1}^{2}}+\frac{k_{2}^{2}-\frac{1}{4}}{x_{2}^{2}}\right)\right] d t\right\}
$$




\section{Polar Coordinates:}

$$
\begin{aligned}
& =\int_{\rho\left(t^{\prime}\right)=\rho^{\prime}}^{\rho\left(t^{\prime \prime}\right)=\rho^{\prime \prime}} \rho \mathcal{D} \rho(t) \int_{\phi\left(t^{\prime}\right)=\phi^{\prime}}^{\phi\left(t^{\prime \prime}\right)=\phi^{\prime \prime}} \mathcal{D} \phi(t) \\
& \quad \times \exp \left\{\frac{\mathrm{i}}{\hbar} \int_{t^{\prime}}^{t^{\prime \prime}}\left[\frac{M}{2}\left(\dot{\rho}^{2}+\rho^{2} \dot{\phi}^{2}-\omega^{2} \rho^{2}\right)-\frac{\hbar^{2}}{2 M \rho^{2}}\left(\frac{k_{1}^{2}-\frac{1}{4}}{\cos ^{2} \phi}+\frac{k_{2}^{2}-\frac{1}{4}}{\sin ^{2} \phi}-\frac{1}{4}\right)\right] d t\right\}
\end{aligned}
$$

\section{Elliptic Coordinates:}

$$
\begin{aligned}
& =\int_{\xi\left(t^{\prime}\right)=\xi^{\prime}}^{\xi\left(t^{\prime \prime}\right)=\xi^{\prime \prime}} \mathcal{D} \xi(t) \int_{\eta\left(t^{\prime}\right)=\eta^{\prime}}^{\eta\left(t^{\prime \prime}\right)=\eta^{\prime \prime}} \mathcal{D} \eta(t) d^{2}\left(\sinh ^{2} \xi+\sin ^{2} \eta\right) \\
& \quad \times \exp \left\{\frac { \mathrm { i } } { \hbar } \int _ { t ^ { \prime } } ^ { t ^ { \prime \prime } } \left[\frac{M}{2} d^{2}\left(\left(\sinh ^{2} \xi+\sin ^{2} \eta\right)\left(\dot{\xi}^{2}+\dot{\eta}^{2}\right)-\omega^{2}\left(\cosh ^{2} \xi \cos ^{2} \eta+\sinh ^{2} \xi \sin ^{2} \eta\right)\right)\right.\right. \\
& \left.\left.\quad-\frac{\hbar^{2}}{2 M d^{2}}\left(\frac{k_{1}^{2}-\frac{1}{4}}{\cosh ^{2} \xi \cos ^{2} \eta}+\frac{k_{2}^{2}-\frac{1}{4}}{\sinh ^{2} \xi \sin ^{2} \eta}\right)\right] d t\right\} \\
& =\int_{\mathbb{R}} \frac{d E}{2 \pi \hbar} \mathrm{e}^{-\mathrm{i} E T / \hbar} \int_{0}^{\infty} d s^{\prime \prime} \int_{\xi\left(s^{\prime \prime}\right)=\xi^{\prime \prime}} \mathcal{D} \xi(s) \int_{\eta\left(s^{\prime \prime}\right)=\eta^{\prime \prime}} \mathcal{D} \eta(s) \\
& \quad \times \exp \left\{\frac { \mathrm { i } } { \hbar } \int _ { 0 } ^ { s ^ { \prime \prime } } \left[\frac{M}{2}\left(\left(\dot{\xi}^{2}+\dot{\eta}^{2}\right)-\omega^{2}\left(\cosh ^{2} \xi \sinh ^{2} \xi+\sin ^{2} \eta \cos ^{2} \eta\right)\right)+E d^{2}\left(\sinh ^{2} \xi+\sin ^{2} \eta\right)\right.\right. \\
& \left.\left.\quad-\frac{\hbar^{2}}{2 M}\left(\left(k_{1}^{2}-\frac{1}{4}\right)\left(\frac{1}{\cos ^{2} \eta}-\frac{1}{\cosh ^{2} \xi}\right)+\left(k_{2}^{2}-\frac{1}{4}\right)\left(\frac{1}{\sin ^{2} \eta}+\frac{1}{\sinh ^{2} \xi}\right)\right)\right] d s\right\}
\end{aligned}
$$

Note that in the last line a pure time-transformation [84 has been performed.

We are going to discuss the path integral (84) in some detail in order to demonstrate the relevant technique. All subsequent path integral evaluations are similarly done and our method remains the same whether polar, parabolic, or other coordinate systems are used.

The path integral solution in cartesian coordinates is straightforward by applying the path integral solution for the radial harmonic oscillator for the coordinates $x_{1,2}$, respectively, c.f. (92, 93). In the case of polar coordinates one first separates the $\phi$-path integration by means of the path integral identity of the Pöschl-Teller potential $(0<\phi<\pi / 2)$ 6, 28, 72]

$$
\begin{aligned}
& \int_{\phi\left(t^{\prime}\right)=\phi^{\prime}}^{\phi\left(t^{\prime \prime}\right)=\phi^{\prime \prime}} \mathcal{D} \phi(t) \exp \left\{\frac{\mathrm{i}}{\hbar} \int_{t^{\prime}}^{t^{\prime \prime}}\left[\frac{M}{2} \dot{\phi}^{2}-\frac{\hbar^{2}}{2 M}\left(\frac{\alpha^{2}-\frac{1}{4}}{\sin ^{2} \phi}+\frac{\beta^{2}-\frac{1}{4}}{\cos ^{2} \phi}\right)\right] d t\right\} \\
& \quad=\sum_{n=0}^{\infty} \mathrm{e}^{-\mathrm{i} E_{n} T / \hbar} \Phi_{n}^{(\alpha, \beta)}\left(\phi^{\prime}\right) \Phi_{n}^{(\alpha, \beta)}\left(\phi^{\prime \prime}\right),
\end{aligned}
$$

and $\Phi_{n}^{(\alpha, \beta)}(\phi)$ denote the normalized Pöschl-Teller wave-functions

$$
\begin{aligned}
\Phi_{n}^{(\alpha, \beta)}(\phi)=[2(\alpha+\beta & \left.+2 l+1) \frac{l ! \Gamma(\alpha+\beta+l+1)}{\Gamma(\alpha+l+1) \Gamma(\beta+l+1)}\right]^{1 / 2} \\
& \times(\sin \phi)^{\alpha+1 / 2}(\cos \phi)^{\beta+1 / 2} P_{n}^{(\alpha, \beta)}(\cos 2 \phi) .
\end{aligned}
$$

The $P_{n}^{(\alpha, \beta)}(x)$ are Jacobi polynomials (49, p.1035). Note that the genuine Pöschl-Teller potentials requires $\alpha, \beta>\frac{1}{2}$. Otherwise one can call it an attractive Pöschl-Teller-like potential. This rule of separation of variables in the path integral consists of the following path integral identity 
55] $\left(\hat{g}=\prod g_{i}^{2},\left\{g_{i}\right\}_{i} \equiv \vec{g} \equiv \vec{g}(\vec{z})\right)$

$$
\begin{aligned}
& \int_{\vec{z}\left(t^{\prime}\right)=\vec{z}^{\prime}}^{z\left(t^{\prime \prime}\right)=\vec{z}^{\prime \prime}} f^{d}(\vec{z}) \sqrt{g(\vec{z})} \mathcal{D} \vec{z}(t) \int_{\vec{x}\left(t^{\prime}\right)=\vec{x}^{\prime}}^{\vec{x}\left(t^{\prime \prime}\right)=\vec{x}^{\prime \prime}} \mathcal{D} \vec{x}(t) \\
& \times \exp \left\{\frac{i}{\hbar} \int_{t^{\prime}}^{t^{\prime \prime}}\left[\frac{M}{2}\left((\vec{g} \cdot \dot{\vec{z}})^{2}+f^{2}(\vec{z}) \dot{\vec{x}}^{2}\right)-\left(\frac{V(\vec{x})}{f^{2}(\vec{z})}+W(\vec{z})+\Delta W(\vec{z})\right)\right] d t\right\} \\
& =\left[f\left(\vec{z}^{\prime}\right) f\left(\vec{z}^{\prime \prime}\right)\right]^{-d / 2} \int d E_{\lambda} \Phi_{\lambda}^{*}\left(\vec{x}^{\prime}\right) \Phi_{\lambda}\left(\vec{x}^{\prime \prime}\right) \int_{\vec{z}\left(t^{\prime \prime}\right)=\vec{z}^{\prime \prime}}^{\vec{z}\left(t^{\prime}\right)=\vec{z}^{\prime \prime}} \sqrt{(\vec{z})} \mathcal{D} \vec{z}(t) \\
& \times \exp \left\{\frac{\mathrm{i}}{\hbar} \int_{t^{\prime}}^{t^{\prime \prime}}\left[\frac{M}{2}(\vec{g} \cdot \dot{\vec{z}})^{2}-W(\vec{z})-\Delta W(\vec{z})-\frac{E_{\lambda}}{f^{2}(\vec{z})}\right] d t\right\}
\end{aligned}
$$

where the $x$-path integration is given by

$$
\int_{\vec{x}\left(t^{\prime}\right)=\vec{x}^{\prime}}^{\vec{x}\left(t^{\prime \prime}\right)=\vec{x}^{\prime \prime}} \mathcal{D} \vec{x}(t) \exp \left\{\frac{\mathrm{i}}{\hbar} \int_{t^{\prime}}^{t^{\prime \prime}}\left[\frac{M}{2} \dot{\vec{x}}^{2}-V(\vec{x})\right] d t\right\}=\int d E_{\lambda} \Phi_{\lambda}^{*}\left(\vec{x}^{\prime}\right) \Phi_{\lambda}\left(\vec{x}^{\prime \prime}\right) \mathrm{e}^{-\mathrm{i} E_{\lambda} T / \hbar} .
$$

The remaining $\rho$-path integration (97) is again of the radial harmonic oscillator type. The radial $1 / \rho^{2}$-dependence must be incorporated into the path integral in terms of a functional weight $\mu_{\lambda}\left[\rho^{2}\right]$ (98) in order to guarantee a proper definition of the corresponding lattice formulation of the radial path integral [59, 119]. As it turns out, this Besselian path integral formulation [70] with the functional weight $\mu_{\lambda}\left[\rho^{2}\right]$ for radial path integrals can be interpreted as an additional potential term, and vice versa [40, 86]. We also want to point out that a similar reasoning must be done in the case of the Pöschl-Teller and modified Pöschl-Teller potential, respectively [41, 53]. Hence we apply the radial harmonic oscillator the path integral solution and its expansion into wave-functions according to $(\lambda$ an arbitrary parameter [29, 48, 113, 119])

$$
\begin{array}{rl}
\int_{r\left(t^{\prime}\right)=r^{\prime}}^{r\left(t^{\prime \prime}\right)=r^{\prime \prime}} \mathcal{D} & r(t) \exp \left[\frac{\mathrm{i}}{\hbar} \int_{t^{\prime}}^{t^{\prime \prime}}\left(\frac{M}{2} \dot{r}^{2}-\hbar^{2} \frac{\lambda^{2}-\frac{1}{4}}{2 M r^{2}}-\frac{M}{2} \omega^{2} r^{2}\right) d t\right] \\
= & \int_{r\left(t^{\prime}\right)=r^{\prime}}^{r\left(t^{\prime \prime}\right)=r^{\prime \prime}} \mu_{\lambda}\left[r^{2}\right] \mathcal{D} r(t) \exp \left[\frac{\mathrm{i} M}{2 \hbar} \int_{t^{\prime}}^{t^{\prime \prime}}\left(\dot{r}^{2}-\omega^{2} r^{2}\right) d t\right] \\
= & \frac{M \omega \sqrt{r^{\prime} r^{\prime \prime}}}{\mathrm{i} \hbar \sin \omega T} \exp \left[-\frac{M \omega}{2 \mathrm{i} \hbar}\left(r^{\prime 2}+r^{\prime \prime 2}\right) \cot \omega T\right] I_{\lambda}\left(\frac{M \omega r^{\prime} r^{\prime \prime}}{\mathrm{i} \hbar \sin \omega T}\right) \\
= & \frac{2 M \omega}{\hbar} \sqrt{r^{\prime} r^{\prime \prime}} \sum_{n=0}^{\infty} \mathrm{e}^{-\mathrm{i} \omega T(2 n+\lambda+1)} \frac{n !}{\Gamma(n+\lambda+1)}\left(\frac{M \omega}{\hbar} r^{\prime} r^{\prime \prime}\right)^{\lambda} \\
& \quad \times \exp \left(-\frac{M \omega}{2 \hbar}\left(r^{\prime 2}+r^{\prime \prime 2}\right)\right) L_{n}^{(\lambda)}\left(\frac{M \omega}{\hbar} r^{\prime 2}\right) L_{n}^{(\lambda)}\left(\frac{M \omega}{\hbar} r^{\prime \prime 2}\right) .
\end{array}
$$

The expansion into the wave-functions has been obtained by means of the Hille-Hardy formula (49], p.1038)

$$
\frac{t^{-\alpha / 2}}{1-t} \exp \left[-\frac{1}{2}(x+y) \frac{1+t}{1-t}\right] I_{\alpha}\left(\frac{2 \sqrt{x y t}}{1-t}\right)=\sum_{n=0}^{\infty} \frac{t^{n} n ! \mathrm{e}^{-\frac{1}{2}(x+y)}}{\Gamma(n+\alpha+1)}(x y)^{\alpha / 2} L_{n}(x) L_{n}(y) .
$$

The $L_{n}(x)$ are Laguerre polynomials ([49], p.1037). We therefore obtain 
$K^{\left(V_{1}\right)}\left(\vec{x}^{\prime \prime}, \vec{x}^{\prime} ; T\right)$

Cartesian Coordinates [29, 48, 113, 119]:

$=\left(\frac{M \omega}{\mathrm{i} \hbar \sin \omega T}\right)^{2} \prod_{i=1}^{2} \sqrt{x_{i}^{\prime} x_{i}^{\prime \prime}} \exp \left[-\frac{M \omega}{2 \mathrm{i} \hbar}\left(x_{i}^{\prime 2}+x_{i}^{\prime \prime 2}\right) \cot \omega T\right] I_{ \pm k_{i}}\left(\frac{M \omega x_{i}^{\prime} x_{i}^{\prime \prime}}{\mathrm{i} \hbar \sin \omega T}\right)$

$=\sum_{n_{1}, n_{2}=0}^{\infty} \mathrm{e}^{-\mathrm{i} E_{N} T / \hbar} \Psi_{n_{1}, n_{2}}\left(x_{1}^{\prime}, x_{2}^{\prime}\right) \Psi_{n_{1}, n_{2}}\left(x_{1}^{\prime \prime}, x_{2}^{\prime \prime}\right)$

Polar Coordinates:

$=\frac{1}{\sqrt{\rho^{\prime} \rho^{\prime \prime}}} \sum_{n=0}^{\infty} \Phi_{n}^{\left( \pm k_{2}, \pm k_{1}\right)}\left(\phi^{\prime}\right) \Phi_{n}^{\left( \pm k_{2}, \pm k_{1}\right)}\left(\phi^{\prime \prime}\right) \int_{\rho\left(t^{\prime}\right)=\rho^{\prime}}^{\rho\left(t^{\prime \prime}\right)=\rho^{\prime \prime}} \mathcal{D} \rho(t) \exp \left\{\frac{\mathrm{i}}{\hbar} \int_{t^{\prime}}^{t^{\prime \prime}}\left[\frac{M}{2}\left(\dot{\rho}^{2}-\omega^{2} \rho^{2}\right)-\hbar^{2} \frac{\lambda^{2}-\frac{1}{4}}{2 M \rho^{2}}\right] d t\right\}$

$=\left(\rho^{\prime} \rho^{\prime \prime}\right)^{-1 / 2} \sum_{n=0}^{\infty} \Phi_{n}^{\left( \pm k_{2}, \pm k_{1}\right)}\left(\phi^{\prime}\right) \Phi_{n}^{\left( \pm k_{2}, \pm k_{1}\right)}\left(\phi^{\prime \prime}\right) \int_{\rho\left(t^{\prime}\right)=\rho^{\prime}}^{\rho\left(t^{\prime \prime}\right)=\rho^{\prime \prime}} \mu_{\lambda}\left[\rho^{2}\right] \mathcal{D} \rho(t) \exp \left[\frac{\mathrm{i} M}{2 \hbar} \int_{t^{\prime}}^{t^{\prime \prime}}\left(\dot{\rho}^{2}-\omega^{2} \rho^{2}\right) d t\right]$

$=\frac{M \omega}{\mathrm{i} \hbar \sin \omega T} \sum_{n=0}^{\infty} \Phi_{n}^{\left( \pm k_{2}, \pm k_{1}\right)}\left(\phi^{\prime}\right) \Phi_{n}^{\left( \pm k_{2}, \pm k_{1}\right)}\left(\phi^{\prime \prime}\right) \exp \left[-\frac{M \omega}{2 \mathrm{i} \hbar}\left({\rho^{\prime 2}}^{2}+{\rho^{\prime \prime 2}}^{2}\right) \cot \omega T\right] I_{\lambda}\left(\frac{M \omega \rho^{\prime} \rho^{\prime \prime}}{\mathrm{i} \hbar \sin \omega T}\right)$

$=\sum_{m, n=0}^{\infty} \mathrm{e}^{-\mathrm{i} E_{N} T / \hbar} \Psi_{m, n}\left(\phi^{\prime}, \rho^{\prime}\right) \Psi_{m, n}\left(\phi^{\prime \prime}, \rho^{\prime \prime}\right)$,

where $\lambda=2 n \pm k_{1} \pm k_{2}+1$. The wave-functions and the energy-spectra are given by

Cartesian Coordinates:

$\Psi_{n_{1}, n_{2}}\left(x_{1}, x_{2}\right)=\frac{2 M \omega}{\hbar} \prod_{i=1}^{2}\left(\frac{M \omega}{\hbar}\right)^{ \pm k_{i} / 2} \sqrt{\frac{n_{i} !}{\Gamma\left(n_{i} \pm k_{i}+1\right)}} x_{i}^{1 / 2 \pm k_{i}} \exp \left(-\frac{M \omega}{2 \hbar} x_{i}^{2}\right) L_{n_{i}}^{\left( \pm k_{i}\right)}\left(\frac{M \omega}{\hbar} x_{i}^{2}\right)$,

$E_{N}=\hbar \omega\left(2 N \pm k_{1} \pm k_{2}+2\right), \quad N=n_{1}+n_{2}$,

Polar Coordinates:

$\Psi_{m, n}(\rho, \phi)=\Phi_{n}^{\left( \pm k_{2}, \pm k_{1}\right)}(\phi) \sqrt{\frac{2 M \omega}{\hbar}}\left(\frac{M \omega}{\hbar} \rho^{2}\right)^{\lambda / 2} \sqrt{\frac{m !}{\Gamma(m+\lambda+1)}} \exp \left(-\frac{M \omega}{2 \hbar} \rho^{2}\right) L_{m}^{(\lambda)}\left(\frac{M \omega}{\hbar} \rho^{2}\right)$,

$E_{N}=\hbar \omega\left(2 N \pm k_{1} \pm k_{2}+2\right), \quad N=m+n$,

and $\Phi_{n}^{(\alpha, \beta)}(\phi)$ of (88) with $\alpha= \pm k_{2}, \beta= \pm k_{1}$. Let us remark that the wave-functions have been normalized in the domain $[0, \infty)$. The positive sign at the $k_{i}$ has to be taken whenever $k_{i} \geq \frac{1}{2}$, i.e. the radial potential term is repulsive at the origin, and the motion takes only place in the domain $x_{i}>0$. If $0<\left|k_{i}\right|<\frac{1}{2}$, i.e. the radial potential term is attractive at the origin, both the positive and the negative sign must be taken into account in the solution. This is indicated by the notion $\pm k_{i}$ in the formulæ. It has also the consequence that for each $k_{i}$ the motion can take place on the entire real line. In the present case this means that in cartesian coordinates we must distinguish four cases: i) $x_{1}, x_{2}>0$, ii) $x_{1}>0, x_{2} \in \mathbb{R}$, iii) $x_{1} \in \mathbb{R}, x_{2}>0$ and iv) $\left(x_{1}, x_{2}\right) \in \mathbb{R}^{2}$. In polar coordinates the same feature is recovered by the observation that the Pöschl-Teller barriers are absent for $\left|k_{i}\right|<\frac{1}{2}$. We will keep this notion in the sequel for all 
following Smorodinsky-Winternitz potentials.

3.1.2. We consider the potential $\left(k_{1,2}>0\right)$

$$
V_{2}(\vec{x})=\frac{M}{2} \omega^{2}\left(4 x^{2}+y^{2}\right)+k_{1} x+\frac{\hbar^{2}}{2 M} \frac{k_{2}^{2}-\frac{1}{4}}{y^{2}},
$$

which is separable in two coordinate systems. For $k_{1}=0$ this potential is also called Holtpotential 66. We have

$$
K^{\left(V_{2}\right)}\left(\vec{x}^{\prime \prime}, \vec{x}^{\prime} ; T\right)
$$

Cartesian Coordinates:

$$
=\int_{\vec{x}\left(t^{\prime}\right)=\vec{x}^{\prime}}^{\vec{x}\left(t^{\prime \prime}\right)=\vec{x}^{\prime \prime}} \mathcal{D} \vec{x}(t) \exp \left\{\frac{\mathrm{i}}{\hbar} \int_{t^{\prime}}^{t^{\prime \prime}}\left[\frac{M}{2}\left(\dot{\vec{x}}^{2}-\omega^{2}\left(4 x^{2}+y^{2}\right)\right)-k_{1} x-\frac{\hbar^{2}}{2 M} \frac{k_{2}^{2}-\frac{1}{4}}{y^{2}}\right] d t\right\}
$$

Parabolic Coordinates:

$$
\begin{aligned}
& =\int_{\eta\left(t^{\prime}\right)=\eta^{\prime}} \mathcal{D} \eta(t) \int_{\xi\left(t^{\prime}\right)=\xi^{\prime}}^{\xi\left(t^{\prime \prime}\right)=\xi^{\prime \prime}} \mathcal{D} \xi(t)\left(\xi^{2}+\eta^{2}\right) \\
& \times \exp \left\{\frac{\mathrm{i}}{\hbar} \int_{t^{\prime}}^{t^{\prime \prime}}\left[\frac{M}{2}\left(\left(\xi^{2}+\eta^{2}\right)\left(\dot{\xi}^{2}+\dot{\eta}^{2}\right)-\omega^{2}\left(\left(\xi^{2}-\eta^{2}\right)^{2}-\xi^{2} \eta^{2}\right)\right)-\frac{k_{1}}{2}\left(\xi^{2}-\eta^{2}\right)-\hbar^{2} \frac{k_{2}^{2}-\frac{1}{4}}{2 M \eta^{2} \xi^{2}}\right] d t\right\} \\
& =\int_{\mathbb{R}} \frac{d E}{2 \pi \hbar} \mathrm{e}^{-\mathrm{i} E T / \hbar} \int_{0}^{\infty} d s^{\prime \prime} \int_{\eta(0)=\eta^{\prime}}^{\eta\left(s^{\prime \prime}\right)=\eta^{\prime \prime}} \mathcal{D} \eta(s) \int_{\xi(0)=\xi^{\prime}}^{\xi\left(s^{\prime \prime}\right)=\xi^{\prime \prime}} \mathcal{D} \xi(s) \\
& \times \exp \left\{\frac { \mathrm { i } } { \hbar } \int _ { 0 } ^ { s ^ { \prime \prime } } \left[\frac{M}{2}\left(\left(\dot{\xi}^{2}+\dot{\eta}^{2}\right)-\omega^{2}\left(\xi^{6}+\eta^{6}\right)\right)\right.\right. \\
& \left.\left.\quad-\frac{k_{1}}{2}\left(\xi^{4}-\eta^{4}\right)+E\left(\xi^{2}+\eta^{2}\right)-\frac{\hbar^{2}}{2 M}\left(\frac{k_{2}^{2}-\frac{1}{4}}{\eta^{2}}+\frac{k_{2}^{2}-\frac{1}{4}}{\xi^{2}}\right)\right] d s\right\} .
\end{aligned}
$$

In the $x$-path integration one makes use of the shifted harmonic oscillator path integral solution 37, 38, and in the $y$-path integration one makes use of the radial harmonic oscillator path integral. The expansion into the wave-functions in $x$ is obtained by means of the Mehler formula (33], p.272)

$$
\mathrm{e}^{-\left(x^{2}+y^{2}\right) / 2} \sum_{n=0}^{\infty} \frac{1}{n !}\left(\frac{z}{2}\right)^{n} H_{n}(x) H_{n}(y)=\frac{1}{\sqrt{1-z^{2}}} \exp \left[\frac{4 x y z-\left(x^{2}+y^{2}\right)\left(1+z^{2}\right)}{2\left(1-z^{2}\right)}\right] .
$$

The $H_{n}(x)$ are Hermite polynomials (44], p.1033). We have for the harmonic oscillator path integral the kernel and its expansion into wave-functions according to (we omit the Maslow indices)

$$
\begin{aligned}
& \int_{x\left(t^{\prime}\right)=x^{\prime}}^{x\left(t^{\prime \prime}\right)=x^{\prime \prime}} \mathcal{D} x(t) \exp \left[\frac{\mathrm{i} M}{2 \hbar} \int_{t^{\prime}}^{t^{\prime \prime}}\left(\dot{x}^{2}-\omega^{2} x^{2}\right) d t\right] \\
& =\left(\frac{M \omega}{2 \pi \mathrm{i} \hbar \sin \omega T}\right)^{1 / 2} \exp \left\{-\frac{M \omega}{2 \mathrm{i} \hbar}\left[\left(x^{\prime 2}+x^{\prime \prime 2}\right) \cot \omega T-2 \frac{x^{\prime} x^{\prime \prime}}{\sin \omega T}\right]\right\} \\
& =\sum_{n=0}^{\infty} \frac{\mathrm{e}^{-\mathrm{i} \omega T(n+1 / 2)}}{2^{n} n !}\left(\frac{M \omega}{\pi \hbar}\right)^{1 / 2} H_{n}\left(\sqrt{\frac{M \omega}{\hbar}} x^{\prime}\right) H_{n}\left(\sqrt{\frac{M \omega}{\hbar}} x^{\prime \prime}\right) \exp \left(-\frac{M \omega}{2 \hbar}\left(x^{\prime 2}+x^{\prime \prime 2}\right)\right) .
\end{aligned}
$$


The $\xi^{4}, \eta^{4}, \xi^{6}, \eta^{6}$-terms make the path integral in parabolic coordinates intractable. For $k_{1}=0$ the anharmonic sextic potential problems in $\xi$ and $\eta$ can only be treated for a zero separation constant, c.f. the appendix. The result in cartesian coordinates has the form $\left(\tilde{x}=x+k_{1} / 4 M \omega^{2}\right)$ 


$$
\begin{aligned}
K^{\left(V_{2}\right)}\left(\vec{x}^{\prime \prime}, \vec{x}^{\prime} ; T\right)= & \left(\frac{M \omega}{2 \pi \mathrm{i} \hbar \sin \omega T}\right)^{1 / 2} \exp \left\{\frac{\mathrm{i} M \omega}{2 \hbar \sin \omega T}\left[\left(\tilde{x}^{2}+\tilde{x}^{\prime \prime 2}\right) \cos \omega T-2 \tilde{x}^{\prime} \tilde{x}^{\prime \prime}\right]\right\} \\
& \times \frac{M \omega}{2 \mathrm{i} \hbar \sin \omega T} \exp \left[-\frac{M \omega}{2 \mathrm{i} \hbar}\left(y^{\prime 2}+y^{\prime \prime 2}\right) \cos \omega T\right] I_{k_{2}}\left(\frac{M \omega y^{\prime} y^{\prime \prime}}{\mathrm{i} \hbar \sin \omega T}\right) \\
= & \sum_{n_{1}, n_{2}=0}^{\infty} \mathrm{e}^{-\mathrm{i} E_{n_{1}, n_{2}} T / \hbar} \Psi_{n_{1}, n_{2}}\left(x^{\prime \prime}, y^{\prime \prime}\right) \Psi_{n_{1}, n_{2}}\left(x^{\prime}, y^{\prime}\right)
\end{aligned}
$$

with the wave-functions and the energy-spectrum given by

$$
\begin{aligned}
& \Psi_{n_{1}, n_{2}}(x, y)= \sqrt{\frac{2 M \omega}{\hbar}}\left(\frac{M \omega}{\hbar}\right)^{ \pm k_{2} / 2} \sqrt{\frac{n_{2} !}{\Gamma\left(n_{2} \pm k_{2}+1\right)}} y^{1 / 2 \pm k_{2}} \exp \left(-\frac{M \omega}{2 \hbar} y^{2}\right) L_{n_{2}}^{\left( \pm k_{2}\right)}\left(\frac{M \omega}{\hbar} y^{2}\right) \\
& \times \sqrt{\sqrt{\frac{2 M \omega}{\pi \hbar}} \frac{1}{2^{n_{1}} n_{1} !} \exp \left(-\frac{M \omega}{\hbar} \tilde{x}^{2}\right) H_{n_{1}}\left(\sqrt{\frac{2 M \omega}{\hbar}} \tilde{x}\right),} \\
& E_{n_{1}, n_{2}}=\hbar \omega\left(n_{1}+2 n_{2} \pm k_{2}+\frac{3}{2}\right)+\frac{k_{1}^{2}}{8 M \omega^{2}} .
\end{aligned}
$$

Note the cases whether $k_{2} \geq \frac{1}{2}$, respectively $0<k_{2}<\frac{1}{2}$.

3.1.3. We consider the potential $\left(k_{1,2}>0\right)$

$$
V_{3}(\vec{x})=-\frac{\alpha}{\sqrt{x^{2}+y^{2}}}+\frac{\hbar^{2}}{4 M} \frac{1}{\sqrt{x^{2}+y^{2}}}\left(\frac{k_{1}^{2}-\frac{1}{4}}{\sqrt{x^{2}+y^{2}}+x}+\frac{k_{2}^{2}-\frac{1}{4}}{\sqrt{x^{2}+y^{2}}-x}\right) .
$$

This potential is separable in polar, parabolic and elliptic II coordinates. A path integral discussion for the pure Coulomb case is due to Inomata [68 and Duru and Kleinert 31. We have

$$
K^{\left(V_{3}\right)}\left(\vec{x}^{\prime \prime}, \vec{x}^{\prime} ; T\right)
$$

\section{Polar Coordinates:}

$$
\begin{aligned}
& =\int_{\rho\left(t^{\prime}\right)=\rho^{\prime}}^{\rho\left(t^{\prime \prime}\right)=\rho^{\prime \prime}} \rho \mathcal{D} \rho(t) \int_{\phi\left(t^{\prime}\right)=\phi^{\prime}}^{\phi\left(t^{\prime \prime}\right)=\phi^{\prime \prime}} \mathcal{D} \phi(t) \\
& \quad \times \exp \left\{\frac{\mathrm{i}}{\hbar} \int_{t^{\prime}}^{t^{\prime \prime}}\left[\frac{M}{2}\left(\dot{\rho}^{2}+\rho^{2} \dot{\phi}^{2}\right)+\frac{\alpha}{\rho}-\frac{\hbar^{2}}{8 M \rho^{2}}\left(\frac{k_{1}^{2}-\frac{1}{4}}{\cos ^{2} \frac{\phi}{2}}+\frac{k_{2}^{2}-\frac{1}{4}}{\sin ^{2} \frac{\phi}{2}}-1\right)\right] d t\right\}
\end{aligned}
$$

Elliptic II Coordinates [95]:

$$
\begin{array}{r}
=\int_{\xi\left(t^{\prime}\right)=\xi^{\prime}}^{\xi\left(t^{\prime \prime}\right)=\xi^{\prime \prime}} \mathcal{D} \xi(t) \int_{\eta\left(t^{\prime}\right)=\eta^{\prime}}^{\eta\left(t^{\prime \prime}\right)=\eta^{\prime \prime}} \mathcal{D} \eta(t) d^{2}\left(\sinh ^{2} \xi+\sin ^{2} \eta\right) \\
\quad \times \exp \left\{\frac { \mathrm { i } } { \hbar } \int _ { t ^ { \prime } } ^ { t ^ { \prime \prime } } \left[\frac{M}{2}\left(\sinh ^{2} \xi^{2}+\sin ^{2} \eta\right)\left(\dot{\xi}^{2}+\dot{\eta}^{2}\right)+\frac{\alpha}{d(\cosh \xi+\cos \eta)}\right.\right. \\
\quad-\frac{\hbar^{2}}{4 M d\left(\cosh ^{2} \xi-\cos ^{2} \eta\right)}\left(\frac{\left(k_{1}^{2}+k_{2}^{2}-\frac{1}{2}\right)+\left(k_{2}^{2}-k_{1}^{2}\right) \cos \eta}{\sin ^{2} \eta}\right. \\
\left.\left.\left.+\frac{\left(k_{1}^{2}+k_{2}^{2}-\frac{1}{2}\right)+\left(k_{2}^{2}-k_{1}^{2}\right) \cosh \xi}{\sinh ^{2} \xi}\right)\right] d t\right\}
\end{array}
$$

Parabolic Coordinates [21]: 


$$
\begin{aligned}
& =\int_{\eta\left(t^{\prime}\right)=\eta^{\prime}}^{\eta\left(t^{\prime \prime}\right)=\eta^{\prime \prime}} \mathcal{D} \eta(t) \int_{\xi\left(t^{\prime}\right)=\xi^{\prime}}^{\xi\left(t^{\prime \prime}\right)=\xi^{\prime \prime}} \mathcal{D} \xi(t)\left(\xi^{2}+\eta^{2}\right) \\
& \quad \times \exp \left\{\frac{\mathrm{i}}{\hbar} \int_{t^{\prime}}^{t^{\prime \prime}}\left[\frac{M}{2}\left(\xi^{2}+\eta^{2}\right)\left(\dot{\xi}^{2}+\dot{\eta}^{2}\right)+\frac{2 \alpha}{\xi^{2}+\eta^{2}}-\frac{\hbar^{2}}{2 M\left(\xi^{2}+\eta^{2}\right)}\left(\frac{k_{1}^{2}-\frac{1}{4}}{\xi^{2}}+\frac{k_{2}^{2}-\frac{1}{4}}{\eta^{2}}\right)\right] d t\right\} .
\end{aligned}
$$

If the domain of the angular variable is $\phi \in(0,2 \pi)$ there are two separated domains (halfplanes) in the case of $k_{1}>\frac{1}{2}$. A rotation of the axis about $\pi / 2$ interchanges the roles of $k_{1}$ and $k_{2}$. In order to evaluate the two path integrals in polar and parabolic coordinates a space-time transformation must be performed. The result in polar coordinates has the form $\left[\kappa=\alpha \sqrt{-M / 2 E} / \hbar, \lambda=m+\left(1 \pm k_{1} \pm k_{2}\right) / 2\right.$, only the radial Green function can be explicitly evaluated]

$$
\begin{aligned}
& G^{\left(V_{3}\right)}\left(\vec{x}^{\prime \prime}, \vec{x}^{\prime} ; E\right)=\frac{\mathrm{i}}{\hbar} \int_{0}^{\infty} d T \mathrm{e}^{\mathrm{i} T E / \hbar} K^{\left(V_{3}\right)}\left(\vec{x}^{\prime \prime}, \vec{x}^{\prime} ; T\right) \\
& =\frac{1}{2}\left(r^{\prime} r^{\prime \prime}\right)^{-1 / 2} \sum_{l=0}^{\infty} \Phi_{n}^{\left( \pm k_{2}, \pm k_{1}\right)}\left(\frac{\phi^{\prime}}{2}\right) \Phi_{n}^{\left( \pm k_{2}, \pm k_{1}\right)}\left(\frac{\phi^{\prime \prime}}{2}\right) \\
& \quad \times \frac{\mathrm{i}}{\hbar} \int_{0}^{\infty} d T \mathrm{e}^{\mathrm{i} T E / \hbar} \int_{\rho\left(t^{\prime}\right)=\rho^{\prime}}^{\rho\left(t^{\prime \prime}\right)=\rho^{\prime \prime}} \mathcal{D} \rho(t) \exp \left[\frac{\mathrm{i}}{\hbar} \int_{t^{\prime}}^{t^{\prime \prime}}\left(\frac{M}{2} \dot{\rho}^{2}+\frac{\alpha}{\rho}-\hbar^{2} \frac{\lambda^{2}-\frac{1}{4}}{2 M \rho^{2}}\right) d t\right] \\
& =\frac{1}{2}\left(\rho^{\prime} \rho^{\prime \prime}\right)^{-1 / 2} \sum_{n=0}^{\infty} \Phi_{n}^{\left( \pm k_{2}, \pm k_{1}\right)}\left(\frac{\phi^{\prime}}{2}\right) \Phi_{n}^{\left( \pm k_{2}, \pm k_{1}\right)}\left(\frac{\phi^{\prime \prime}}{2}\right) \\
& \quad \times \frac{1}{\hbar} \sqrt{-\frac{M}{2 E}} \frac{\Gamma\left(\frac{1}{2}+\lambda-\kappa\right)}{\Gamma(2 \lambda+1)} W_{\kappa, \lambda}\left(\sqrt{-8 M E} \frac{\rho_{>}}{\hbar}\right) M_{\kappa, \lambda}\left(\sqrt{-8 M E} \frac{\rho_{<}}{\hbar}\right) \\
& =\sum_{n=0}^{\infty}\left\{\sum_{m=0}^{\infty} \frac{\Psi_{n, m}^{*}\left(\rho^{\prime}, \phi^{\prime}\right) \Psi_{n, m}\left(\rho^{\prime \prime}, \phi^{\prime \prime}\right)}{E_{m}-E}+\int_{\mathbb{R}} d p \frac{\Psi_{p, m}^{*}\left(\rho^{\prime}, \phi^{\prime}\right) \Psi_{p, m}^{\alpha)}\left(\rho^{\prime \prime}, \phi^{\prime \prime}\right)}{\hbar^{2} p^{2} / 2 M-E}\right\} .
\end{aligned}
$$

In the $\phi$-path integration the path integral solution of the Pöschl-Teller potential has been used. In the radial path integration the path integral solution for the radial Coulomb potential was used (Chetouani and Hammann [19], Duru and Kleinert [30, 31], and Refs. [56, 118]), and the Green function is expanded according to (Kratzer potential, $\kappa=\alpha \sqrt{-M / 2 E} / \hbar$ )

$$
\begin{aligned}
\frac{\mathrm{i}}{\hbar} \int_{0}^{\infty} d T \mathrm{e}^{\mathrm{i} T E / \hbar} \int_{x\left(t^{\prime}\right)=x^{\prime}}^{x\left(t^{\prime \prime}\right)=x^{\prime \prime}} \mathcal{D} x(t) \exp \left[\frac{\mathrm{i}}{\hbar} \int_{t^{\prime}}^{t^{\prime \prime}}\left(\frac{M}{2} \dot{x}^{2}+\frac{\alpha}{|x|}-\frac{\hbar^{2}}{2 M} \frac{\lambda^{2}-\frac{1}{4}}{x^{2}}\right) d t\right] \\
\quad=\frac{1}{\hbar} \sqrt{-\frac{M}{2 E} \frac{\Gamma\left(\frac{1}{2}+\lambda-\kappa\right)}{\Gamma(2 \lambda+1)} W_{\kappa, \lambda}\left(\sqrt{-8 M E} \frac{x_{>}}{\hbar}\right) M_{\kappa, \lambda}\left(\sqrt{-8 M E} \frac{x_{<}}{\hbar}\right)} \\
=\sum_{n=0}^{\infty} \frac{\Psi_{n}\left(x^{\prime}\right) \Psi_{n}\left(x^{\prime \prime}\right)}{E_{n}-E}+\int_{\mathbb{R}} d p \frac{\Psi_{p}^{*}\left(x^{\prime}\right) \Psi_{p}\left(x^{\prime \prime}\right)}{E_{p}-E} .
\end{aligned}
$$

$x_{<,>}$denotes the smaller/larger of $x^{\prime}, x^{\prime \prime}$. The $W_{\kappa, \lambda}(x)$ and $M_{\kappa, \lambda}(x)$ are Whittaker functions (49], p.1059). The bound state wave-functions and the energy-spectrum of the Kratzer potential are given by $\left(a=\hbar^{2} / M \alpha\right)$

$$
\Psi_{n}(x)=\frac{1}{n+\lambda+\frac{1}{2}}\left[\frac{n !}{a \Gamma(n+2 \lambda+1)}\right]^{1 / 2}
$$




$$
\begin{gathered}
\times\left(\frac{2 x}{a\left(n+\lambda+\frac{1}{2}\right)}\right)^{\lambda+1 / 2} \exp \left[-\frac{x}{a(n+1)}\right] L_{n}^{(2 \lambda)}\left(\frac{2 x}{a(n+1)}\right), \\
E_{n}=-\frac{M \alpha^{2}}{2 \hbar^{2}\left(n+\lambda+\frac{1}{2}\right)^{2}},
\end{gathered}
$$

and for the continuous spectrum with $E_{p}=p^{2} \hbar^{2} / 2 M$ one has

$$
\Psi_{p}(x)=\frac{\Gamma\left(\frac{1}{2}+\lambda-\mathrm{i} / a p\right)}{\sqrt{2 \pi} \Gamma(2 \lambda+1)} \exp \left(\frac{\pi}{2 a p}\right) M_{\mathrm{i} / a p, \lambda}(-2 \mathrm{i} p x) .
$$

Therefore the polar coordinate wave-functions and the energy-spectrum of our potential problem are given by $\left(a=M / \alpha \hbar^{2}\right)$ :

$$
\begin{aligned}
\Psi_{n, m}(\rho, \phi)= & 2^{-1 / 2} \Phi_{n}^{\left( \pm k_{2}, \pm k_{1}\right)}\left(\frac{\phi}{2}\right)\left[\frac{2 m !}{a^{2}\left(m+\lambda+\frac{1}{2}\right)^{3} \Gamma(m+2 \lambda+1)}\right]^{1 / 2} \\
& \times\left(\frac{2 \rho}{a\left(m+\lambda+\frac{1}{2}\right)}\right)^{\lambda} \exp \left(-\frac{\rho}{a\left(m+\lambda+\frac{1}{2}\right)}\right) L_{m}^{(2 \lambda)}\left(\frac{2 \rho}{a\left(m+\lambda+\frac{1}{2}\right)}\right) \\
E_{n}= & -\frac{M \alpha^{2}}{2 \hbar^{2}\left(m+\lambda+\frac{1}{2}\right)^{2}} \\
\Psi_{n, p}(\rho, \phi)= & 2^{-1 / 2} \Phi_{n}^{\left( \pm k_{2}, \pm k_{1}\right)}\left(\frac{\phi}{2}\right) \frac{\Gamma\left(\frac{1}{2}+\lambda-\mathrm{i} / a p\right)}{\sqrt{2 \pi \rho} \Gamma(2 \lambda+1)} \exp \left(\frac{\pi}{2 a p}\right) M_{\mathrm{i} / a p, \lambda}(-2 \mathrm{i} p \rho)
\end{aligned}
$$

The $\Phi_{n}^{\left( \pm k_{2}, \pm k_{1}\right)}(\phi / 2)$ are the Pöschl-Teller wave-functions (88).

In parabolic coordinates we obtain by performing a time transformation and applying the path integral solution of the radial harmonic oscillator (92) in the $\xi$ and $\eta$ variable, respectively $(\omega=\sqrt{-2 E / M})$

$$
\begin{aligned}
& \frac{\mathrm{i}}{\hbar} \int_{0}^{\infty} d T \mathrm{e}^{\mathrm{i} T E / \hbar} K^{\left(V_{3}\right)}\left(\vec{x}^{\prime \prime}, \vec{x}^{\prime} ; T\right) \\
& =\int_{0}^{\infty} d s^{\prime \prime} e^{2 \mathrm{i} \alpha s^{\prime \prime} / \hbar} \int_{\eta(0)=\eta^{\prime}}^{\eta\left(s^{\prime \prime}\right)=\eta^{\prime \prime}} \mathcal{D} \eta(s) \int_{\xi(0)=\xi^{\prime}}^{\xi\left(s^{\prime \prime}\right)=\xi^{\prime \prime}} \mathcal{D} \xi(s) \\
& \quad \times \exp \left\{\frac{\mathrm{i}}{\hbar} \int_{0}^{s^{\prime \prime}}\left[\frac{M}{2}\left(\dot{\xi}^{2}+\dot{\eta}^{2}\right)+E\left(\xi^{2}+\eta^{2}\right)-\frac{\hbar^{2}}{2 M}\left(\frac{k_{1}^{2}-\frac{1}{4}}{\xi^{2}}+\frac{k_{2}^{2}-\frac{1}{4}}{\eta^{2}}\right)\right] d t\right\} \\
& =\left(\frac{M}{\mathrm{i} \hbar}\right)^{2} \sqrt{\xi^{\prime} \xi^{\prime \prime} \eta^{\prime} \eta^{\prime \prime}} \int_{0}^{\infty} \frac{\omega^{2} d s^{\prime \prime}}{\sin ^{2} \omega s^{\prime \prime}} \mathrm{e}^{2 \mathrm{i} \alpha s^{\prime \prime} / \hbar} \\
& \quad \times \exp \left[-\frac{M \omega}{2 \mathrm{i} \hbar}\left(\xi^{\prime 2}+\xi^{\prime \prime 2}+\eta^{\prime 2}+\eta^{\prime \prime 2}\right) \cot \omega s^{\prime \prime}\right] I_{k_{1}}\left(\frac{M \omega \xi^{\prime} \xi^{\prime \prime}}{\mathrm{i} \hbar \sin \omega s^{\prime \prime}}\right) I_{k_{2}}\left(\frac{M \omega \eta^{\prime} \eta^{\prime \prime}}{\mathrm{i} \hbar \sin \omega s^{\prime \prime}}\right) \\
& =\sum_{n_{1}, n_{2}=0}^{\infty} \frac{\Psi_{n_{1}, n_{2}}^{*}\left(\xi^{\prime}, \eta^{\prime}\right) \Psi_{n_{1}, n_{2}}\left(\xi^{\prime \prime}, \eta^{\prime \prime}\right)}{E_{n_{1}, n_{2}}-E}+\int_{0}^{\infty} d p \int_{\mathbb{R}} d \zeta \frac{\Psi_{p, \zeta}^{*}\left(\xi^{\prime}, \eta^{\prime}\right) \Psi_{p, \zeta}\left(\xi^{\prime \prime}, \eta^{\prime \prime}\right)}{E_{p}-E},
\end{aligned}
$$

where are $\left(N=n_{1}+n_{2}+\frac{1}{2}\left( \pm k_{1} \pm k_{2}\right)+1, a=\hbar^{2} / M \alpha\right)$

$$
\begin{aligned}
& \Psi_{n_{1}, n_{2}}(\xi, \eta)= {\left[\frac{2}{a^{2} N^{3}} \cdot \frac{n_{1} ! n_{2} !}{\Gamma\left(n_{1} \pm k_{1}+1\right) \Gamma\left(n_{2} \pm k_{2}+1\right)}\right]^{1 / 2} } \\
& \times\left(\frac{\xi}{a N}\right)^{ \pm k_{1} / 2}\left(\frac{\eta}{a N}\right)^{ \pm k_{2} / 2} \exp \left(-\frac{\xi^{2}+\eta^{2}}{2 a N}\right) L_{n_{1}}^{\left( \pm k_{1}\right)}\left(\frac{\xi^{2}}{a N}\right) L_{n_{2}}^{\left( \pm k_{2}\right)}\left(\frac{\eta^{2}}{a N}\right) \\
& E_{n_{1}, n_{2}}=-\frac{M \alpha^{2}}{\hbar^{2} N^{2}}
\end{aligned}
$$




$$
\begin{aligned}
& \Psi_{p, \zeta}(\xi, \eta)=\frac{\Gamma\left[\frac{1 \pm k_{2}}{2}+\mathrm{i}(1 / a+\zeta) / 2 p\right] \Gamma\left[\frac{1 \pm k_{1}}{2}+\mathrm{i}(1 / a-\zeta) / 2 p\right]}{2 \pi \sqrt{\xi \eta} \Gamma\left(1 \pm k_{2}\right) \Gamma\left(1 \pm k_{1}\right)} \mathrm{e}^{\pi / 2 a p} \\
& \quad \times M_{-\mathrm{i}(1 / a+\zeta) / 2 p, \pm k_{1} / 2}\left(-\mathrm{i} p \xi^{2}\right) M_{-\mathrm{i}(1 / a-\zeta) / 2 p, \pm k_{2} / 2}\left(-\mathrm{i} p \eta^{2}\right) \\
& E_{p}=\frac{\hbar^{2}}{2 M} p^{2} .
\end{aligned}
$$

In order to extract the discrete spectrum one applies (c.f. [20, 56, 58]) the Mehler formula [33]. For the continuous spectrum one applies the following dispersion relation (c.f. [8], p.158, [34], p.414, 49, p.884)

$$
\begin{aligned}
& \frac{1}{\sin \alpha} \exp [-(x+y) \cot \alpha] I_{2 \mu}\left(\frac{2 \sqrt{x y}}{\sin \alpha}\right) \\
& =\frac{1}{2 \pi \sqrt{x y}} \int_{\mathbb{R}} \frac{\Gamma\left(\frac{1}{2}+\mu+\mathrm{i} p\right) \Gamma\left(\frac{1}{2}+\mu-\mathrm{i} p\right)}{\Gamma^{2}(1+2 \mu)} \mathrm{e}^{-2 \alpha p+\pi p} M_{+\mathrm{i} p, \mu}(-2 \mathrm{i} x) M_{-\mathrm{i} p, \mu}(+2 \mathrm{i} y) d p
\end{aligned}
$$

and performs the variable substitution $\left(\nu_{1}, \nu_{2}\right) \mapsto\left(p_{\xi}, p_{\eta}\right) \mapsto[(1 / a+\zeta) / 2 p,(1 / a-\zeta) / 2 p]$. $\zeta$ is the parabolic separation constant. In elliptic II coordinates $K^{\left(V_{3}\right)}$ is not explicitly soluble and we obtain only the path integral identity

$$
\begin{aligned}
& K^{\left(V_{3}\right)}\left(\vec{x}^{\prime \prime}, \vec{x}^{\prime} ; T\right) \\
& =\int_{\mathbb{R}} \frac{d E}{2 \pi \hbar} \mathrm{e}^{-\mathrm{i} E T / \hbar} \int_{0}^{\infty} d s^{\prime \prime} \int_{\xi(0)=\xi^{\prime}}^{\xi\left(s^{\prime \prime}\right)=\xi^{\prime \prime}} \mathcal{D} \xi(s) \int_{\eta(0)=\eta^{\prime}}^{\eta\left(s^{\prime \prime}\right)=\eta^{\prime \prime}} \mathcal{D} \eta(s) \\
& \times \exp \left\{\frac { \mathrm { i } } { \hbar } \int _ { 0 } ^ { s ^ { \prime \prime } } \left[\frac{M}{2}\left(\dot{\xi}^{2}+\dot{\eta}^{2}\right)+E d^{2}\left(\cosh ^{2} \xi-\cos ^{2} \eta\right)+\alpha d(\cosh \xi+\cos \eta)\right.\right. \\
& \left.\left.-\frac{\hbar^{2}}{2 M}\left(\frac{\left(k_{1}^{2}-\frac{1}{4}\right)(1-\cos \eta)+\left(k_{2}^{2}-\frac{1}{4}\right)(1+\cos \eta)}{\sin ^{2} \eta}\right)\right] d s\right\} . \\
& \left.\left.\quad+\frac{\left(k_{1}^{2}-\frac{1}{4}\right)(1-\cosh \xi)+\left(k_{2}^{2}-\frac{1}{4}\right)(1+\cosh \xi)}{\sinh ^{2} \xi}\right)\right]
\end{aligned}
$$

No further evaluation is possible.

3.1.4. We consider the potential $\left(\beta_{1,2} \in \mathbb{R}\right)$

$$
V_{4}(\vec{x})=-\frac{\alpha}{\rho}+\sqrt{\frac{2}{\rho}}\left(\beta_{1} \cos \frac{\phi}{2}+\beta_{2} \sin \frac{\phi}{2}\right) .
$$

This potential is only separable in mutually orthogonal parabolic coordinates and again a time transformation must be performed. We obtain $[\omega=\sqrt{-2 E / M}$, only the Green function can be explicitly evaluated, $\left.(\tilde{\xi}, \tilde{\eta})=\left(\xi-\beta_{1} / E, \eta-\beta_{2} / E\right)\right]$

$$
\begin{aligned}
& \frac{\mathrm{i}}{\hbar} \int_{0}^{\infty} d T \mathrm{e}^{\mathrm{i} T E / \hbar} \int_{\vec{x}\left(t^{\prime}\right)=\vec{x}^{\prime}}^{\vec{x}\left(t^{\prime \prime}\right)=\vec{x}^{\prime \prime}} \mathcal{D} \vec{x}(t) \\
& \quad \times \exp \left\{\frac{\mathrm{i}}{\hbar} \int_{t^{\prime}}^{t^{\prime \prime}}\left[\frac{M}{2}\left(\dot{\rho}^{2}+\rho^{2} \dot{\phi}^{2}\right)+\frac{\alpha}{\rho}+\frac{\alpha}{\rho}+\sqrt{\frac{2}{\rho}}\left(\beta_{1} \cos \frac{\phi}{2}+\beta_{2} \sin \frac{\phi}{2}\right)\right] d t\right\} \\
& =\frac{\mathrm{i}}{\hbar} \int_{0}^{\infty} d T \mathrm{e}^{\mathrm{i} T E / \hbar} \int_{\eta\left(t^{\prime}\right)=\eta^{\prime}}^{\eta\left(t^{\prime \prime}\right)=\eta^{\prime \prime}} \mathcal{D} \eta(t) \int_{\xi\left(t^{\prime}\right)=\xi^{\prime}}^{\left.t^{\prime \prime}\right)=\xi^{\prime \prime}} \mathcal{D} \xi(t)\left(\xi^{2}+\eta^{2}\right)
\end{aligned}
$$




$$
\begin{aligned}
& \times \exp \left\{\frac{\mathrm{i}}{\hbar} \int_{t^{\prime}}^{t^{\prime \prime}}\left[\frac{M}{2}\left(\xi^{2}+\eta^{2}\right)\left(\dot{\xi}^{2}+\dot{\eta}^{2}\right)+2 \frac{\alpha-\left(\beta_{1} \xi+\beta_{2} \eta\right)}{\xi^{2}+\eta^{2}}\right] d t\right\} \\
& =\int_{0}^{\infty} d s^{\prime \prime} \mathrm{e}^{2 \mathrm{i} \alpha s^{\prime \prime} / \hbar} \int_{\eta(0)=\eta^{\prime}}^{\eta\left(s^{\prime \prime}\right)=\eta^{\prime \prime}} \mathcal{D} \eta(s) \int_{\xi(0)=\xi^{\prime}}^{\xi\left(s^{\prime \prime}\right)=\xi^{\prime \prime}} \mathcal{D} \xi(s) \\
& \times \exp \left\{\frac{\mathrm{i}}{\hbar} \int_{0}^{s^{\prime \prime}}\left[\frac{M}{2}\left(\dot{\xi}^{2}+\dot{\eta}^{2}\right)+E\left(\xi^{2}+\eta^{2}\right)-2\left(\beta_{1} \xi+\beta_{2} \eta\right)\right] d t\right\} \\
& =\int_{0}^{\infty} d s^{\prime \prime} \frac{M \omega}{\pi \mathrm{i} \hbar \sin \omega s^{\prime \prime}} \cosh \left(\frac{M \omega\left(\tilde{\xi}^{\prime} \tilde{\xi}^{\prime \prime}+\tilde{\eta}^{\prime} \tilde{\eta}^{\prime \prime}\right)}{\mathrm{i} \hbar \sin \omega s^{\prime \prime}}\right) \\
& \times \exp \left[\frac{2 \mathrm{i} \alpha s^{\prime \prime}}{\hbar}-\frac{\mathrm{i}}{\hbar} \frac{\beta_{1}^{2}+\beta_{2}^{2}}{E} s^{\prime \prime}+\frac{\mathrm{i} M \omega}{2 \hbar}\left(\tilde{\xi}^{\prime 2}+\tilde{\xi}^{\prime \prime 2}+\tilde{\eta}^{2}+\tilde{\eta}^{\prime \prime 2}\right) \cot \omega s^{\prime \prime}\right] \\
& =\sum_{n_{1}, n_{2}=0}^{\infty} \frac{\Psi_{n_{1}, n_{2}}\left(\xi^{\prime}, \eta^{\prime}\right) \Psi_{n_{1}, n_{2}}\left(\xi^{\prime \prime}, \eta^{\prime \prime}\right)}{E_{n_{1}, n_{2}}-E}+\sum_{e, o} \int_{\mathbb{R}} d \zeta \int_{\mathbb{R}} d p \frac{\Psi_{p, \zeta}^{(e, o) *}\left(\xi^{\prime}, \eta^{\prime}\right) \Psi_{p, \zeta}^{(e, o)}\left(\xi^{\prime \prime}, \eta^{\prime \prime}\right)}{\hbar^{2} p^{2} / 2 M-E},
\end{aligned}
$$

and $\sum_{e, o}$ denotes the summation over even and odd states, respectively; the bound state energylevels are determined by $\left(N=n_{1}+n_{2}+1, n_{1}, n_{2} \in \mathbb{N}_{0}, \omega_{N}=\sqrt{-2 E_{N} / M}\right)$

$$
\omega_{N}^{3}-\frac{2 \alpha}{N \hbar} \omega_{N}^{2}-2 \frac{\beta_{1}^{2}+\beta_{2}^{2}}{M N \hbar}=0
$$

For $\alpha>0$ the discriminante of this cubic equation shows that there is one real root $\omega_{N}$ for each $N$ such that the bound state energy-levels $E_{N}$ are given by

$$
\begin{aligned}
E_{N} & =E_{n_{1}, n_{2}}=-\frac{M}{2} \omega_{N}^{2}, \quad \omega_{N}=y_{N}+\frac{2 \alpha}{3 \hbar N}, \quad y_{N}=u_{1}+u_{2} \\
u_{1,2} & =\sqrt[3]{\left(\frac{2 \alpha}{3 \hbar N}\right)^{3}+\frac{\beta_{1}^{2}+\beta_{2}^{2}}{M N \hbar} \mp \sqrt{\left(\frac{\beta_{1}^{2}+\beta_{2}^{2}}{M N \hbar}\right)^{2}+2 \frac{\beta_{1}^{2}+\beta_{2}^{2}}{M N \hbar}\left(\frac{2 \alpha}{3 \hbar N}\right)^{3}}} .
\end{aligned}
$$

The corresponding bound-state wave-functions have the form

$$
\begin{aligned}
\Psi_{n_{1}, n_{2}}(\xi, \eta)= & \sqrt{\frac{M}{\pi \hbar}} \cdot \frac{4}{n_{1} ! n_{2} ! 2^{n_{1}+n_{2}}}\left(\lim _{E \rightarrow E_{N}} \frac{-\left(\frac{M}{2} \omega_{N}^{2}+E\right) M \omega_{N}^{4} / N}{\omega^{3}-\frac{2 \alpha}{N \hbar} \omega^{2}-2 \frac{\beta_{1}^{2}+\beta_{2}^{2}}{M N \hbar}}\right)^{1 / 2} \\
& \times \exp \left[-\frac{M \omega_{N}}{2 \hbar}\left(\tilde{\xi}^{2}+\tilde{\eta}^{2}\right)\right] H_{n_{1}}\left(\sqrt{\frac{M \omega_{N}}{\hbar}} \tilde{\xi}\right) H_{n_{2}}\left(\sqrt{\frac{M \omega_{N}}{\hbar}} \tilde{\eta}\right) .
\end{aligned}
$$

$N=n_{1}+n_{2}$ must be chosen in such a way that $N=n_{1}+n_{2}$ is an even number [130]. The continuous functions $\Psi_{p, \zeta}^{(e, o)}(\xi, \eta)$ are given by $\left[\tilde{a}=\hbar^{2} / M\left(\alpha-m\left(\beta_{1}^{2}+\beta_{2}^{2}\right) / \hbar^{2} p^{2}\right)\right]$

$$
\begin{aligned}
\Psi_{p, \zeta}^{(e, o)}(\xi, \eta)=\frac{\mathrm{e}^{\pi / 2 a p}}{\sqrt{2} 4 \pi^{2}}\left(\begin{array}{c}
\Gamma\left[\frac{1}{4}+\frac{\mathrm{i}}{2 p}(1 / \tilde{a}+\zeta)\right] E_{-\frac{1}{2}+\frac{\mathrm{i}}{p}(1 / \tilde{a}+\zeta)}^{(0)}\left(\mathrm{e}^{-\mathrm{i} \pi / 4} \sqrt{2 p} \tilde{\xi}\right) \\
\left.\Gamma\left[\frac{3}{4}+\frac{\mathrm{i}}{2 p}(1 / \tilde{a}+\zeta)\right] E_{-\frac{1}{2}+\frac{\mathrm{i}}{p}(1 / \tilde{a}+\zeta)}^{\left(\mathrm{e}^{-\mathrm{i} \pi / 4} \sqrt{2 p} \tilde{\xi}\right)}\right)
\end{array}\right) \\
\times\left(\begin{array}{c}
\Gamma\left[\frac{1}{4}+\frac{\mathrm{i}}{2 p}(1 / \tilde{a}-\zeta)\right] E_{-\frac{1}{2}+\frac{\mathrm{i}}{p}(1 / \tilde{a}-\zeta)}^{(0)}\left(\mathrm{e}^{-\mathrm{i} \pi / 4} \sqrt{2 p} \tilde{\eta}\right) \\
\Gamma\left[\frac{3}{4}+\frac{\mathrm{i}}{2 p}(1 / \tilde{a}-\zeta)\right] E_{-\frac{1}{2}+\frac{\mathrm{i}}{p}(1 / \tilde{a}-\zeta)}^{(1)}\left(\mathrm{e}^{-\mathrm{i} \pi / 4} \sqrt{2 p} \tilde{\eta}\right)
\end{array}\right) .
\end{aligned}
$$

For the determination of the continuous spectrum one uses the dispersion relation ([49], p.896)

$$
\begin{aligned}
\int_{c-\mathrm{i} \infty}^{c+\mathrm{i} \infty}\left[D_{\nu}(x) D_{-\nu-1}(\mathrm{i} y)+D_{\nu}(-x) D_{-\nu-1}(-\mathrm{i} y)\right] \frac{t^{-\nu-1} d \nu}{\sin (-\nu \pi)} \\
=2 \mathrm{i} \sqrt{\frac{2 \pi}{1+t^{2}}} \exp \left(\frac{1-t^{2}}{1+t^{2}} \cdot \frac{x^{2}+y^{2}}{4}+\frac{\mathrm{i} t x y}{1+t^{2}}\right),
\end{aligned}
$$


and performs the variable substitution $\left(\nu_{1}, \nu_{2}\right) \mapsto\left(p_{\xi}, p_{\eta}\right) \mapsto[(1 / \tilde{a}+\zeta) / 2 p,(1 / \tilde{a}-\zeta) / 2 p]$. $\zeta$ is the parabolic separation constant. The $D_{\nu}(z)$ are parabolic cylinder functions, and the $E_{\nu}^{(0)}(z)$ and $E_{\nu}^{(1)}(z)$ are even and odd parabolic cylinder functions [8], respectively.

Note that in the evaluation of the path integral one has to take into account that by using the linearly shifted harmonic oscillator solution for the $\xi$ - and $\eta$-variable, respectively, one actually uses a double covering of the original $(x, y) \in \mathbb{R}^{2}$-plane, i.e. $\vec{u} \equiv(\tilde{\xi}, \tilde{\eta}) \in \mathbb{R}^{2}$. Furthermore we have taken into account that our mapping is of the "square-root" type which gives rise to a sign ambiguity. "Thus, if one considers all paths in the complex $z=x+\mathrm{i} y$-plane from $z^{\prime}$ to $z^{\prime \prime}$, they will be mapped into two different classes of paths in the $\vec{u}$-plane: Those which go from $\vec{u}^{\prime}$ to $\vec{u}^{\prime \prime}$ and those going from $\vec{u}^{\prime}$ to $-\vec{u}^{\prime \prime}$. In the cut complex $z$-plane for the function $|\vec{u}|=\sqrt{|z|}$ these are the paths passing an even or odd number of times through the square root from $|z|=0$ and $|z|=-\infty$. We may choose the $\vec{u}^{\prime}$ corresponding to the initial $z^{\prime}$ to lie on the first sheet i.e. in the right half $\vec{u}$-plane). The final $\vec{u}^{\prime \prime}$ can be in the right as well as the left half-plane and all paths on the $z$-plane go over into paths from $\vec{u}^{\prime}$ to $\vec{u}^{\prime \prime}$ and those from $\vec{u}^{\prime}$ to $-\vec{u}^{\prime \prime} " 31$. Thus the two contributions arise in (140).

\subsection{Three-Dimensional Maximally Super-Integrable Smorodinsky-Winternitz Po- tentials.}

We discuss in this subsection the five three-dimensional maximally super-integrable potentials. They are characterized by having five functionally independent integrals of motion. In the sequel $\vec{x}$ denotes a three-dimensional coordinate: $\vec{x}=(x, y, z) \equiv\left(x_{1}, x_{2}, x_{3}\right)$.

In table 3 we list the three-dimensional maximally super-integrable Smorodinsky-Winternitz potentials together with the separating coordinate systems (where the italized coordinates systems were not mentioned in [35]). The cases where an explicit path integration is possible are underlined.

3.2.1. We consider the three-dimensional potential $\left(k_{1,2,3}>0\right)$

$$
V_{1}(\vec{x})=\frac{M}{2} \omega^{2} \vec{x}^{2}+\frac{\hbar^{2}}{2 M}\left(\frac{k_{1}^{2}-\frac{1}{4}}{x^{2}}+\frac{k_{2}^{2}-\frac{1}{4}}{y^{2}}+\frac{k_{3}^{2}-\frac{1}{4}}{z^{2}}\right) .
$$

Note furthermore that the pure three-dimensional harmonic oscillator [73] has the same separating coordinate systems as $V_{1}(\vec{x})$ and therefore the additional centrifugal terms do not spoil this property.

We formulate the corresponding path integral in the coordinate systems which separate the path integral for this potential problem. We have

$$
K^{\left(V_{1}\right)}\left(\vec{x}^{\prime \prime}, \vec{x}^{\prime} ; T\right)
$$

Cartesian Coordinates:

$$
\int_{\vec{x}\left(t^{\prime}\right)=\vec{x}^{\prime}}^{\vec{x}\left(t^{\prime \prime}\right)=\vec{x}^{\prime \prime}} \mathcal{D} \vec{x}(t) \exp \left\{\frac{\mathrm{i}}{\hbar} \int_{t^{\prime}}^{t^{\prime \prime}}\left[\frac{M}{2}\left(\dot{\vec{x}}^{2}-\omega^{2} \vec{x}^{2}\right)-\frac{\hbar^{2}}{2 M} \sum_{i=1}^{3} \frac{k_{i}^{2}-\frac{1}{4}}{x_{i}^{2}}\right] d t\right\}
$$

Spherical Coordinates:

$$
\begin{aligned}
& =\int_{r\left(t^{\prime}\right)=r^{\prime}}^{r\left(t^{\prime \prime}\right)=r^{\prime \prime}} r^{2} \mathcal{D} r(t) \int_{\theta\left(t^{\prime}\right)=\theta^{\prime}}^{\theta\left(t^{\prime \prime}\right)=\theta^{\prime \prime}} \sin \theta \mathcal{D} \theta(t) \int_{\phi\left(t^{\prime}\right)=\phi^{\prime}}^{\phi\left(t^{\prime \prime}\right)=\phi^{\prime \prime}} \mathcal{D} \phi(t) \\
& \quad \times \exp \left\{\frac { \mathrm { i } } { \hbar } \int _ { t ^ { \prime } } ^ { t ^ { \prime \prime } } \left[\frac{M}{2}\left(\dot{r}^{2}+r^{2} \dot{\theta}^{2}+r^{2} \sin ^{2} \theta \dot{\phi}^{2}-\omega^{2} r^{2}\right)\right.\right.
\end{aligned}
$$




$$
\left.\left.-\frac{\hbar^{2}}{2 M r^{2}}\left(\frac{1}{\sin ^{2} \theta}\left(\frac{k_{1}^{2}-\frac{1}{4}}{\cos ^{2} \phi}+\frac{k_{2}^{2}-\frac{1}{4}}{\sin ^{2} \phi}-\frac{1}{4}\right)+\frac{k_{3}^{2}-\frac{1}{4}}{\cos ^{2} \theta}-\frac{1}{4}\right)\right] d t\right\}
$$


Table 3: The three-dimensional maximally super-integrable potentials

\begin{tabular}{|c|c|}
\hline Potential $V(x, y, z)$ & Coordinate System \\
\hline$V_{1}=\frac{M}{2} \omega^{2} \vec{x}^{2}+\frac{\hbar^{2}}{2 M}\left(\frac{k_{1}^{2}-\frac{1}{4}}{x^{2}}+\frac{k_{2}^{2}-\frac{1}{4}}{y^{2}}+\frac{k_{3}^{2}-\frac{1}{4}}{z^{2}}\right)$ & $\begin{array}{l}\text { Cartesian } \\
\text { Spherical } \\
\text { Circular Polar } \\
\text { Circular Elliptic } \\
\text { Conical } \\
\text { Oblate Spheroidal } \\
\text { Prolate Spheroidal } \\
\text { Ellipsoidal }\end{array}$ \\
\hline $\begin{array}{l}\omega=0, \quad k_{3}^{2}-1 / 4=0 \\
\omega=0, \quad k_{1}^{2}-1 / 4=0\end{array}$ & $\begin{array}{l}\text { Paraboloidal } \\
\text { Parabolic } \\
\underline{\text { Circular Parabolic }}\end{array}$ \\
\hline$V_{2}=\frac{M}{2} \omega^{2}\left(x^{2}+y^{2}+4 z^{2}\right)+\frac{\hbar^{2}}{2 M}\left(\frac{k_{1}^{2}-\frac{1}{4}}{x^{2}}+\frac{k_{2}^{2}-\frac{1}{4}}{y^{2}}\right)$ & $\begin{array}{l}\text { Cartesian } \\
\text { Parabolic } \\
\frac{\text { Circular Polar }}{\text { Circular Elliptic }}\end{array}$ \\
\hline$V_{3}=-\frac{\alpha}{\sqrt{x^{2}+y^{2}+z^{2}}}+\frac{\hbar^{2}}{2 M}\left(\frac{k_{1}^{2}-\frac{1}{4}}{x^{2}}+\frac{k_{2}^{2}-\frac{1}{4}}{y^{2}}\right)$ & $\begin{array}{l}\text { Conical } \\
\frac{\text { Spherical }}{\text { Parabolic }} \\
\text { Prolate Spheroidal II }\end{array}$ \\
\hline $\begin{aligned} V_{4} & =\frac{\hbar^{2}}{2 M}\left(\frac{k_{1}^{2} x}{y^{2} \sqrt{x^{2}+y^{2}}}+\frac{k_{2}^{2}-\frac{1}{4}}{y^{2}}+\frac{k_{3}^{2}-\frac{1}{4}}{z^{2}}\right) \\
& =\frac{\hbar^{2}}{2 M}\left[\frac{1}{\sqrt{x^{2}+y^{2}}}\left(\frac{\beta_{1}^{2}-\frac{1}{4}}{\sqrt{x^{2}+y^{2}}+x}+\frac{\beta_{2}^{2}-\frac{1}{4}}{\sqrt{x^{2}+y^{2}}-x}\right)+\frac{k_{3}^{2}-\frac{1}{4}}{z^{2}}\right] \\
\beta_{1}^{2} & =\frac{1}{2}\left(k_{2}^{2}+k_{1}^{2}+\frac{1}{4}\right), \quad \beta_{2}^{2}=\frac{1}{2}\left(k_{2}^{2}-k_{1}^{2}+\frac{1}{4}\right)\end{aligned}$ & $\begin{array}{l}\text { Spherical } \\
\text { Circular Elliptic II } \\
\text { Circular Parabolic } \\
\text { Circular Polar }\end{array}$ \\
\hline $\begin{aligned} V_{5} & =\frac{\hbar^{2}}{2 M}\left(\frac{k_{1}^{2} x}{y^{2} \sqrt{x^{2}+y^{2}}}+\frac{k_{2}^{2}-\frac{1}{4}}{y^{2}}\right)-k_{3} z \\
& =\frac{\hbar^{2}}{2 M \sqrt{x^{2}+y^{2}}}\left(\frac{\beta_{1}^{2}-\frac{1}{4}}{\sqrt{x^{2}+y^{2}}+x}+\frac{\beta_{2}^{2}-\frac{1}{4}}{\sqrt{x^{2}+y^{2}}-x}\right)-k_{3} z \\
\beta_{1}^{2} & =\frac{1}{2}\left(k_{2}^{2}+k_{1}^{2}+\frac{1}{4}\right), \quad \beta_{2}^{2}=\frac{1}{2}\left(k_{2}^{2}-k_{1}^{2}+\frac{1}{4}\right)\end{aligned}$ & $\begin{array}{l}\text { Circular Polar } \\
\text { Circular Elliptic II } \\
\text { Circular Parabolic } \\
\text { Parabolic }\end{array}$ \\
\hline
\end{tabular}

Circular Polar Coordinates:

$$
\begin{aligned}
& =\int_{z\left(t^{\prime}\right)=z^{\prime}}^{z\left(t^{\prime \prime}\right)=z^{\prime \prime}} \mathcal{D} z(t) \int_{\rho\left(t^{\prime}\right)=\rho^{\prime}}^{\rho\left(t^{\prime \prime}\right)=\rho^{\prime \prime}} \rho \mathcal{D} \rho(t) \int_{\phi\left(t^{\prime}\right)=\phi^{\prime}}^{\phi\left(t^{\prime \prime}\right)=\phi^{\prime \prime}} \mathcal{D} \phi(t) \\
& \quad \times \exp \left\{\frac{\mathrm{i}}{\hbar} \int_{t^{\prime}}^{t^{\prime \prime}}\left[\frac{M}{2}\left(\dot{\rho}^{2}+\rho^{2} \dot{\phi}^{2}-\omega^{2} \rho^{2}\right)-\frac{\hbar^{2}}{2 M}\left(\frac{k_{1}^{2}-\frac{1}{4}}{\rho^{2} \cos ^{2} \phi}+\frac{k_{2}^{2}-\frac{1}{4}}{\rho^{2} \sin ^{2} \phi}+\frac{k_{3}^{2}-\frac{1}{4}}{z^{2}}-\frac{1}{4 \rho^{2}}\right)\right] d t\right\}
\end{aligned}
$$


Circular Elliptic Coordinates:

$$
\begin{gathered}
=\int_{z\left(t^{\prime}\right)=z^{\prime}}^{z\left(t^{\prime \prime}\right)=z^{\prime \prime}} \mathcal{D} z(t) \int_{\xi\left(t^{\prime}\right)=\xi^{\prime}}^{\xi\left(t^{\prime \prime}\right)=\xi^{\prime \prime}} \mathcal{D} \xi(t) \int_{\eta\left(t^{\prime}\right)=\eta^{\prime}}^{\eta\left(t^{\prime \prime}\right)=\eta^{\prime \prime}} \mathcal{D} \eta(t) d^{2}\left(\sinh ^{2} \xi+\sin ^{2} \eta\right) \\
\times \exp \left\{\frac { \mathrm { i } } { \hbar } \int _ { t ^ { \prime } } ^ { t ^ { \prime \prime } } \left[\frac{M}{2}\left(d^{2}\left(\sinh ^{2} \xi+\sin ^{2} \eta\right)\left(\dot{\xi}^{2}+\dot{\eta}^{2}\right)+\dot{z}^{2}\right)\right.\right. \\
-\frac{M}{2} \omega^{2}\left(d^{2}\left(\cosh ^{2} \xi \cos ^{2} \eta+\sinh ^{2} \xi \sin ^{2} \eta\right)+z^{2}\right) \\
\left.\left.-\frac{\hbar^{2}}{2 M}\left(\frac{k_{1}^{2}-\frac{1}{4}}{d^{2} \cosh ^{2} \xi \cos ^{2} \eta}+\frac{k_{2}^{2}-\frac{1}{4}}{d^{2} \sinh ^{2} \xi \sin ^{2} \eta}+\frac{k_{3}^{2}-\frac{1}{4}}{z^{2}}\right)\right] d t\right\}
\end{gathered}
$$

Conical Coordinates:

$$
\begin{gathered}
\int_{r\left(t^{\prime}\right)=r^{\prime}}^{r\left(t^{\prime \prime}\right)=r^{\prime \prime}} r^{2} \mathcal{D} r(t) \int_{\alpha\left(t^{\prime}\right)=\alpha^{\prime}}^{\alpha\left(t^{\prime \prime}\right)=\alpha^{\prime \prime}} \mathcal{D} \alpha(t) \int_{\beta\left(t^{\prime}\right)=\beta^{\prime}}^{\beta\left(t^{\prime \prime}\right)=\beta^{\prime \prime}} \mathcal{D} \beta(t)\left(k^{2} \mathrm{cn}^{2} \alpha+k^{\prime 2} \mathrm{cn}^{2} \beta\right) \\
\times \exp \left\{\frac { \mathrm { i } } { \hbar } \int _ { t ^ { \prime } } ^ { t ^ { \prime \prime } } \left[\frac{M}{2}\left(\dot{r}^{2}+r^{2}\left(k^{2} \mathrm{cn}^{2} \alpha+k^{\prime 2} \mathrm{cn}^{2} \beta\right)\left(\dot{\alpha}^{2}+\dot{\beta}^{2}\right)-\omega^{2} r^{2}\right)\right.\right. \\
\left.\left.\quad-\frac{\hbar^{2}}{2 M r^{2}}\left(\frac{k_{1}^{2}-\frac{1}{4}}{\operatorname{sn}^{2} \alpha \mathrm{dn}^{2} \beta}+\frac{k_{2}^{2}-\frac{1}{4}}{\operatorname{cn}^{2} \alpha \mathrm{cn}^{2} \beta}+\frac{k_{3}^{2}-\frac{1}{4}}{\operatorname{dn}^{2} \alpha \mathrm{sn}^{2} \beta}\right)\right] d t\right\}
\end{gathered}
$$

Oblate Spheroidal Coordinates $\left(\lambda_{1}=2 n \pm k_{1} \pm k_{2}+1\right)$ :

$$
\begin{aligned}
& \bar{\mu}\left(t^{\prime \prime}\right)=\bar{\mu}^{\prime \prime} \quad \bar{\nu}\left(t^{\prime \prime}\right)=\bar{\nu}^{\prime \prime} \quad \phi\left(t^{\prime \prime}\right)=\phi^{\prime \prime} \\
& =\int_{\bar{\mu}\left(t^{\prime}\right)=\bar{\mu}^{\prime}} \mathcal{D} \bar{\mu}(t) \int_{\bar{\nu}\left(t^{\prime}\right)=\bar{\nu}^{\prime}} \mathcal{D} \bar{\nu}(t) \bar{d}^{3}\left(\cosh ^{2} \bar{\mu}-\sin ^{2} \bar{\nu}\right) \cosh \bar{\mu} \sin \bar{\nu} \int_{\phi\left(t^{\prime}\right)=\phi^{\prime}} \mathcal{D} \phi(t) \\
& \times \exp \left\{\frac { \mathrm { i } } { \hbar } \int _ { t ^ { \prime } } ^ { t ^ { \prime \prime } } \left[\frac { M } { 2 } \overline { d } ^ { 2 } \left(\left(\cosh ^{2} \bar{\mu}-\sin ^{2} \bar{\nu}\right)\left(\dot{\bar{\mu}}^{2}+\dot{\bar{\nu}}^{2}\right)+\cosh ^{2} \bar{\mu} \sin ^{2} \bar{\nu} \dot{\phi}^{2}\right.\right.\right. \\
& \left.-\omega^{2}\left(\cosh ^{2} \bar{\mu} \sin ^{2} \bar{\nu}+\sinh ^{2} \bar{\mu} \cos ^{2} \bar{\nu}\right)\right) \\
& \left.\left.-\frac{\hbar^{2}}{2 M \bar{d}^{2}}\left(\frac{1}{\cosh ^{2} \bar{\mu} \sin ^{2} \bar{\nu}}\left(\frac{k_{1}^{2}-\frac{1}{4}}{\cos ^{2} \phi}+\frac{k_{2}^{2}-\frac{1}{4}}{\sin ^{2} \phi}-\frac{1}{4}\right)+\frac{k_{3}^{2}-\frac{1}{4}}{\sinh ^{2} \bar{\mu} \cos ^{2} \bar{\nu}}\right)\right] d t\right\} \\
& =\left(\bar{d}^{2} \cosh \bar{\mu}^{\prime} \cosh \bar{\mu}^{\prime \prime} \sin \bar{\nu}^{\prime} \sin \bar{\nu}^{\prime \prime}\right)^{-1 / 2} \sum_{n=0}^{\infty} \Phi_{n}^{\left( \pm k_{2}, \pm k_{1}\right)}\left(\phi^{\prime}\right) \Phi_{n}^{\left( \pm k_{2}, \pm k_{1}\right)}\left(\phi^{\prime \prime}\right) \\
& \times \int_{\mathbb{R}} \frac{d E}{2 \pi \hbar} \mathrm{e}^{-\mathrm{i} E T / \hbar} \int_{0}^{\infty} d s^{\prime \prime} \int_{\bar{\mu}(0)=\bar{\mu}^{\prime}}^{\bar{\mu}\left(s^{\prime \prime}\right)=\bar{\mu}^{\prime \prime}} \mathcal{D} \bar{\mu}(s) \int_{\bar{\nu}(0)=\bar{\nu}^{\prime}}^{\bar{\nu}\left(s^{\prime \prime}\right)=\bar{\nu}^{\prime \prime}} \mathcal{D} \bar{\nu}(s) \\
& \times \exp \left\{\frac { \mathrm { i } } { \hbar } \int _ { 0 } ^ { s ^ { \prime \prime } } \left[\frac{M}{2}\left(\left(\dot{\bar{\mu}}^{2}+\dot{\bar{\nu}}^{2}\right)-\omega^{2}\left(\cosh ^{2} \bar{\mu} \sinh ^{2} \bar{\mu}+\sin ^{2} \bar{\nu} \cos ^{2} \bar{\nu}\right)\right)+E \bar{d}^{2}\left(\cosh ^{2} \bar{\mu}-\sin ^{2} \bar{\nu}\right)\right.\right. \\
& \left.\left.-\frac{\hbar^{2}}{2 M}\left(\left(\lambda_{1}^{2}-\frac{1}{4}\right)\left(\frac{1}{\cos ^{2} \bar{\nu}}-\frac{1}{\sinh ^{2} \bar{\mu}}\right)+\left(k_{3}^{2}-\frac{1}{4}\right)\left(\frac{1}{\sin ^{2} \bar{\nu}}-\frac{1}{\cosh ^{2} \bar{\mu}}\right)\right)\right] d s\right\}
\end{aligned}
$$

Prolate Spheroidal Coordinates:

$$
\begin{aligned}
& =\int_{\mu\left(t^{\prime}\right)=\mu^{\prime}}^{\mu\left(t^{\prime \prime}\right)=\mu^{\prime \prime}} \mathcal{D} \mu(t) \int_{\nu\left(t^{\prime}\right)=\nu^{\prime}}^{\nu\left(t^{\prime \prime}\right)=\nu^{\prime \prime}} \mathcal{D} \nu(t) d^{3}\left(\sinh ^{2} \mu+\sin ^{2} \nu\right) \sinh \mu \sin \nu \int_{\phi\left(t^{\prime}\right)=\phi^{\prime}}^{\phi\left(t^{\prime \prime}\right)=\phi^{\prime \prime}} \mathcal{D} \phi(t) \\
& \quad \times \exp \left\{\frac { \mathrm { i } } { \hbar } \int _ { t ^ { \prime } } ^ { t ^ { \prime \prime } } \left[\frac { M } { 2 } d ^ { 2 } \left(\left(\sinh ^{2} \mu+\sin ^{2} \nu\right)\left(\dot{\mu}^{2}+\dot{\nu}^{2}\right)+\sinh ^{2} \mu \sin ^{2} \nu \dot{\phi}^{2}\right.\right.\right.
\end{aligned}
$$




$$
\begin{aligned}
& \left.-\omega^{2}\left(\sinh ^{2} \mu \sin ^{2} \nu+\cosh ^{2} \mu \cos ^{2} \nu\right)\right) \\
& \left.\left.-\frac{\hbar^{2}}{2 M d^{2}}\left(\frac{1}{\sinh ^{2} \mu \sin ^{2} \nu}\left(\frac{k_{1}^{2}-\frac{1}{4}}{\cos ^{2} \phi}+\frac{k_{2}^{2}-\frac{1}{4}}{\sin ^{2} \phi}-\frac{1}{4}\right)+\frac{k_{3}^{2}-\frac{1}{4}}{\cosh ^{2} \mu \cos ^{2} \nu}\right)\right] d t\right\}
\end{aligned}
$$

Ellipsoidal Coordinates:

$$
\begin{aligned}
& =\int_{\rho_{1}\left(t^{\prime}\right)=\rho_{1}^{\prime}}^{\rho_{1}\left(t^{\prime \prime}\right)=\rho_{1}^{\prime \prime}} \mathcal{D} \rho_{1}(t) \int_{\rho_{2}\left(t^{\prime}\right)=\rho_{2}^{\prime}}^{\rho_{2}\left(t^{\prime \prime}\right)=\rho_{2}^{\prime \prime}} \mathcal{D} \rho_{2}(t) \int_{\rho_{3}\left(t^{\prime}\right)=\rho_{3}^{\prime}}^{\rho_{3}\left(t^{\prime \prime}\right)=\rho_{3}^{\prime \prime}} \mathcal{D} \rho_{3}(t) \frac{\left(\rho_{2}-\rho_{1}\right)\left(\rho_{3}-\rho_{2}\right)\left(\rho_{3}-\rho_{1}\right)}{8 \sqrt{-P\left(\rho_{1}\right) P\left(\rho_{2}\right) P\left(\rho_{3}\right)}} \\
& \quad \times \exp \left\{\frac{\mathrm{i}}{\hbar} \sum_{i=1}^{3} \int_{t^{\prime}}^{t^{\prime \prime}}\left[\frac{M}{2} g_{\rho_{i} \rho_{i}} \dot{\rho}_{i}^{2}-\frac{1}{\prod_{i \neq j}\left(\rho_{i}-\rho_{j}\right)}\left(\frac{M \omega^{2}}{2} P\left(\rho_{i}\right)+\frac{\hbar^{2}}{2 M} A\left(\rho_{i}\right)\right)-\Delta V_{P F}\left(\rho_{i}\right)\right] d t\right\} .
\end{aligned}
$$

In this example we have explicitly written down the separated $(\bar{\xi}, \bar{\eta})$-path integrations in the case of the oblate spheroidal coordinates. The case of the prolate spheroidal coordinates is, of course, similar. For the separation in the ellipsoidal coordinates $A(\rho)$ denotes $\left(a_{i k}=a_{i}-a_{k}\right)$

$$
A(\rho)=a_{31} a_{21} \frac{k_{1}^{2}-\frac{1}{4}}{\rho-a_{1}}+a_{12} a_{32} \frac{k_{2}^{2}-\frac{1}{4}}{\rho-a_{2}}+a_{13} a_{23} \frac{k_{3}^{2}-\frac{1}{4}}{\rho-a_{3}} .
$$

The separation of variables then is performed by means of (80). In the case of the conical coordinates the separation of variables in the path integral becomes obvious if we consider for fixed $R$ the identity

$$
\begin{aligned}
& \int_{\alpha\left(t^{\prime}\right)=\alpha^{\prime}}^{\alpha\left(t^{\prime \prime}\right)=\alpha^{\prime \prime}} \mathcal{D} \alpha(t) \int_{\beta\left(t^{\prime}\right)=\beta^{\prime}}^{\beta\left(t^{\prime \prime}\right)=\beta^{\prime \prime}} \mathcal{D} \beta(t)\left(k^{2} \mathrm{cn}^{2} \alpha+k^{\prime 2} \mathrm{cn}^{2} \beta\right) \\
& \times \exp \left\{\frac { \mathrm { i } } { \hbar } \int _ { t ^ { \prime } } ^ { t ^ { \prime \prime } } \left[\frac{M}{2} R^{2}\left(k^{2} \mathrm{cn}^{2} \alpha+{k^{\prime}}^{2} \operatorname{cn}^{2} \beta\right)\left(\dot{\alpha}^{2}+\dot{\beta}^{2}\right)\right.\right. \\
& \left.\left.-\frac{\hbar^{2}}{2 M R^{2}}\left(\frac{k_{1}^{2}-\frac{1}{4}}{\operatorname{sn}^{2} \alpha \operatorname{dn}^{2} \beta}+\frac{k_{2}^{2}-\frac{1}{4}}{\operatorname{cn}^{2} \alpha \operatorname{cn}^{2} \beta}+\frac{k_{3}^{2}-\frac{1}{4}}{\operatorname{dn}^{2} \alpha \operatorname{sn}^{2} \beta}\right)\right] d t\right\} \\
& =\int_{\mathbb{R}} \frac{d E}{2 \pi \hbar} \mathrm{e}^{-\mathrm{i} E T / \hbar} \int_{0}^{\infty} d s^{\prime \prime} \int_{\alpha(0)=\alpha^{\prime}}^{\alpha\left(s^{\prime \prime}\right)=\alpha^{\prime \prime}} \mathcal{D} \alpha(s) \int_{\beta(0)=\beta^{\prime}}^{\beta\left(s^{\prime \prime}\right)=\beta^{\prime \prime}} \mathcal{D} \beta(s) \\
& \times \exp \left\{\frac { \mathrm { i } } { \hbar } \int _ { 0 } ^ { s ^ { \prime \prime } } \left[\frac{M}{2} R^{2}\left(\dot{\alpha}^{2}+\dot{\beta}^{2}\right)-\frac{\hbar^{2}}{2 M R^{2}}\left(\left(k_{1}^{2}-\frac{1}{4}\right)\left(\frac{1}{\mathrm{sn}^{2} \alpha}-\frac{k^{2}}{\operatorname{dn}^{2} \beta}\right)\right.\right.\right. \\
& \left.\left.\left.+\left(k_{2}^{2}-\frac{1}{4}\right)\left(\frac{k^{2}}{\mathrm{cn}^{2} \beta}+\frac{k^{\prime 2}}{\mathrm{cn}^{2} \alpha}\right)+\left(k_{3}^{2}-\frac{1}{4}\right)\left(\frac{1}{\operatorname{sn}^{2} \beta}-\frac{k^{\prime 2}}{\operatorname{dn}^{2} \alpha}\right)\right)\right] d s\right\} \text {. }
\end{aligned}
$$

We find that the path integral for this potential can be explicitly evaluated in the cartesian, circular polar and spherical coordinate system [10]. The others cannot be explicitly evaluated, but are however connected through the above path integral identities. We obtain (c.f. for path integral discussions for special cases of $k_{1,2,3}$ e.g. Carpio-Bernido and Bernido [10, 12])

$$
K^{\left(V_{1}\right)}\left(\vec{x}^{\prime \prime}, \vec{x}^{\prime} ; T\right)
$$

Cartesian Coordinates:

$$
=\left(\frac{M \omega}{\mathrm{i} \hbar \sin \omega T}\right)^{3} \prod_{i=1}^{3} \sqrt{x_{i}^{\prime} x_{i}^{\prime \prime}} \exp \left[-\frac{M \omega}{2 \mathrm{i} \hbar}\left(x_{i}^{\prime 2}+x_{i}^{\prime \prime 2}\right) \cot \omega T\right] I_{k_{i}}\left(\frac{M \omega x_{i}^{\prime} x_{i}^{\prime \prime}}{\mathrm{i} \hbar \sin \omega T}\right)
$$


$=\sum_{n_{1}, n_{2}, n_{3}=0}^{\infty} \mathrm{e}^{-\mathrm{i} E_{N} T / \hbar} \Psi_{n_{1}, n_{2}, n_{3}}\left(x_{1}^{\prime \prime}, x_{2}^{\prime \prime}, x_{3}^{\prime \prime}\right) \Psi_{n_{1}, n_{2}, n_{3}}\left(x_{1}^{\prime}, x_{2}^{\prime}, x_{3}^{\prime}\right)$

Circular Polar Coordinates $\left(\lambda_{1}=2 n \pm k_{1} \pm k_{2}+1\right)$ :

$$
\begin{aligned}
= & \left(\frac{M \omega}{\mathrm{i} \hbar \sin \omega T}\right)^{2} \sqrt{z^{\prime} z^{\prime \prime}} \exp \left[-\frac{M \omega}{2 \mathrm{i} \hbar}\left(z^{\prime 2}+z^{\prime \prime 2}\right) \cot \omega T\right] I_{ \pm k_{3}}\left(\frac{M \omega z^{\prime} z^{\prime \prime}}{\mathrm{i} \hbar \sin \omega T}\right) \\
& \times \sum_{n=0}^{\infty} \Phi_{n}^{\left( \pm k_{2}, \pm k_{1}\right)}\left(\phi^{\prime}\right) \Phi_{n}^{\left( \pm k_{2}, \pm k_{1}\right)}\left(\phi^{\prime \prime}\right) \exp \left[-\frac{M \omega}{2 \mathrm{i} \hbar}\left(\rho^{\prime 2}+\rho^{\prime \prime 2}\right) \cot \omega T\right] I_{\lambda_{1}}\left(\frac{M \omega \rho^{\prime} \rho^{\prime \prime}}{\mathrm{i} \hbar \sin \omega T}\right) \\
= & \sum_{n, m, n_{z}=0}^{\infty} \mathrm{e}^{-\mathrm{i} E_{N} T / \hbar} \Psi_{n, m, n_{z}}\left(\phi^{\prime}, \rho^{\prime}, z^{\prime}\right) \Psi_{n, m, n_{z}}\left(\phi^{\prime \prime}, \rho^{\prime \prime}, z^{\prime \prime}\right)
\end{aligned}
$$

Spherical Coordinates $\left(\lambda_{2}=2 m+\lambda_{1} \pm k_{3}+1\right)$ :

$$
\begin{aligned}
= & \left(r^{\prime} r^{\prime \prime} \sin \theta^{\prime} \sin \theta^{\prime \prime}\right)^{-1 / 2} \sum_{n=0}^{\infty} \Phi_{n}^{\left( \pm k_{2}, \pm k_{1}\right)}\left(\phi^{\prime \prime}\right) \Phi_{n}^{\left( \pm k_{2}, \pm k_{1}\right)}\left(\phi^{\prime}\right) \sum_{m=0}^{\infty} \Phi_{m}^{\left(\lambda_{1}, \pm k_{3}\right)}\left(\theta^{\prime \prime}\right) \Phi_{m}^{\left(\lambda_{1}, \pm k_{3}\right)}\left(\theta^{\prime}\right) \\
& \times \frac{M \omega}{\mathrm{i} \hbar \sin \omega T} \exp \left[-\frac{M \omega}{2 \mathrm{i} \hbar}\left(r^{\prime 2}+r^{\prime \prime 2}\right) \cot \omega T\right] I_{\lambda_{2}}\left(\frac{M r^{\prime} r^{\prime \prime}}{\mathrm{i} \hbar \sin \omega T}\right) \\
= & \sum_{l, n, m=0}^{\infty} \mathrm{e}^{-\mathrm{i} E_{N} T / \hbar} \Psi_{l, n, m}\left(\theta^{\prime}, \phi^{\prime}, r^{\prime}\right) \Psi_{l, n, m}\left(\theta^{\prime \prime}, \phi^{\prime \prime}, r^{\prime \prime}\right),
\end{aligned}
$$

with the wave-functions and the energy-spectra given by ( $N$ is the principal quantum number)

\section{Cartesian Coordinates:}

$$
\begin{aligned}
& \Psi_{n_{1}, n_{2}, n_{3}}\left(x_{1}, x_{2}, x_{3}\right)=\left(\frac{2 M \omega}{\hbar}\right)^{3 / 2} \prod_{i=1}^{3}\left(\frac{M \omega}{\hbar}\right)^{ \pm k_{i} / 2} \\
& \times \sqrt{\frac{n_{i} !}{\Gamma\left(n_{i} \pm k_{i}+1\right)}} x_{i}^{1 / 2 \pm k_{i}} \exp \left(-\frac{M \omega}{2 \hbar} x_{i}^{2}\right) L_{n_{i}}^{\left( \pm k_{i}\right)}\left(\frac{M \omega}{\hbar} x_{i}^{2}\right), \\
& E_{N}=\hbar \omega\left(2 N+\sum_{i=1}^{3}\left(1 \pm k_{i}\right)\right), \quad N=n_{1}+n_{2}+n_{3},
\end{aligned}
$$

Circular Polar Coordinates $\left(\lambda_{1}=2 n \pm k_{1} \pm k_{2}+1\right)$ :

$$
\begin{aligned}
& \Psi_{n, m, n_{z}}(\phi, \rho, z)=\Phi_{n}^{\left( \pm k_{2}, \pm k_{1}\right)}(\phi) \sqrt{\frac{2 M \omega}{\hbar}}\left(\frac{M \omega}{\hbar}\right)^{ \pm k_{3} / 2} \\
& \times \sqrt{\frac{n_{z} !}{\Gamma\left(n_{z} \pm k_{3}+1\right)}} z^{1 / 2 \pm k_{3}} \exp \left(-\frac{M \omega}{2 \hbar} z^{2}\right) L_{n_{z}}^{\left( \pm k_{3}\right)}\left(\frac{M \omega}{\hbar} z^{2}\right) \\
& \times \sqrt{\frac{2 M \omega}{\hbar}}\left(\frac{M \omega}{\hbar} \rho^{2}\right)^{\lambda_{1} / 2} \sqrt{\frac{m !}{\Gamma\left(m+\lambda_{1}+1\right)}} \exp \left(-\frac{M \omega}{2 \hbar} \rho^{2}\right) L_{m}^{\left(\lambda_{1}\right)}\left(\frac{M \omega}{\hbar} \rho^{2}\right), \\
& E_{N}=\hbar \omega\left(2 N+\sum_{i=1}^{3}\left(1 \pm k_{i}\right)\right), \quad N=m+n+n_{z},
\end{aligned}
$$

Spherical Coordinates $\left(\lambda_{2}=2 m+\lambda_{1} \pm k_{3}+1\right)$ :

$$
\begin{aligned}
& \Psi_{l, n, m}(\theta, \phi, r)=(r \sin \theta)^{-1 / 2} \Phi_{n}^{\left( \pm k_{2}, \pm k_{1}\right)}(\phi) \Phi_{m}^{\left(\lambda_{1}, \pm k_{3}\right)}(\theta) \\
& \times \sqrt{\frac{2 M \omega}{\hbar}}\left(\frac{M \omega}{\hbar} r^{2}\right)^{\lambda_{2} / 2} \sqrt{\frac{l !}{\Gamma\left(l+\lambda_{2}+1\right)}} \exp \left(-\frac{M \omega}{2 \hbar} r^{2}\right) L_{l}^{\left(\lambda_{2}\right)}\left(\frac{M \omega}{\hbar} r^{2}\right), \\
& E_{N}=\hbar \omega\left(2 N+\sum_{i=1}^{3}\left(1 \pm k_{i}\right)\right), \quad N=n+m+l,
\end{aligned}
$$

and $\Phi_{n}^{\left( \pm k_{2}, \pm k_{1}\right)}(\phi)$ and $\Phi_{m}^{\left(\lambda_{1}, \pm k_{3}\right)}(\theta)$ denote the wave-functions (88), c.f. Calogero [9] and Evans 
[35, 36] for the Schrödinger approach. Note that our solution in circular polar coordinates is new. The $D$-dimensional generalization of this potential is also maximally super-integrable [36], and it is immediately obvious that it is at least separable (and exactly soluble) in cartesian and spherical coordinates (and any combination of circular spherical subsystems).

Let us furthermore consider the two following special cases of the potential $V_{1}(\vec{x})$ where $\omega=0$. Here we have a continuous spectrum instead of a discrete one.

(i) $k_{3}^{2}-\frac{1}{4}=0$ : We have $\left(\lambda=2 n \pm k_{1} \pm k_{2}+1\right)$

$$
K^{\left(V_{1}\right)}\left(\vec{x}^{\prime \prime}, \vec{x}^{\prime} ; T\right)
$$

Paraboloidal Coordinates:

$$
\begin{aligned}
& =\int_{\eta_{1}\left(t^{\prime}\right)=\eta_{1}^{\prime}}^{\eta_{1}\left(t^{\prime \prime}\right)=\eta_{1}^{\prime \prime}} \mathcal{D} \eta_{1}(t) \int_{\eta_{2}\left(t^{\prime}\right)=\eta_{2}^{\prime}}^{\eta_{2}\left(t^{\prime \prime}\right)=\eta_{2}^{\prime \prime}} \mathcal{D} \eta_{2}(t) \int_{\eta_{3}\left(t^{\prime}\right)=\eta_{3}^{\prime}}^{\eta_{3}\left(t^{\prime \prime}\right)=\eta_{3}^{\prime \prime}} \mathcal{D} \eta_{3}(t) \frac{\left(\eta_{2}-\eta_{1}\right)\left(\eta_{3}-\eta_{2}\right)\left(\eta_{3}-\eta_{1}\right)}{8 \sqrt{-P\left(\eta_{1}\right) P\left(\eta_{2}\right) P\left(\eta_{3}\right)}} \\
& \quad \times \exp \left\{\frac{\mathrm{i}}{\hbar} \sum_{i=1}^{3} \int_{t^{\prime}}^{t^{\prime \prime}}\left[\frac{M}{2} g_{\eta_{i} \eta_{i}} \dot{\eta}_{i}^{2}-\frac{\hbar^{2}}{2 M} \frac{B\left(\eta_{i}\right)}{\prod_{i \neq j}\left(\eta_{i}-\eta_{j}\right)}-\Delta V_{P F}\left(\eta_{i}\right)\right] d t\right\}
\end{aligned}
$$

Parabolic Coordinates:

$$
\begin{aligned}
& =\int_{\eta\left(t^{\prime}\right)=\eta^{\prime}}^{\eta\left(t^{\prime \prime}\right)=\eta^{\prime \prime}} \mathcal{D} \eta(t) \int_{\xi\left(t^{\prime}\right)=\xi^{\prime}}^{\xi\left(t^{\prime \prime}\right)=\xi^{\prime \prime}} \mathcal{D} \xi(t)\left(\xi^{2}+\eta^{2}\right) \xi \eta \int_{\phi\left(t^{\prime}\right)=\phi^{\prime}}^{\phi\left(t^{\prime \prime}\right)=\phi^{\prime \prime}} \mathcal{D} \phi(t) \\
& \quad \times \exp \left\{\frac{\mathrm{i}}{\hbar} \int_{t^{\prime}}^{t^{\prime \prime}}\left[\frac{M}{2}\left(\left(\xi^{2}+\eta^{2}\right)\left(\dot{\xi}^{2}+\dot{\eta}^{2}\right)+\xi^{2} \eta^{2} \dot{\phi}^{2}\right)-\frac{\hbar^{2}}{2 M \xi^{2} \eta^{2}}\left(\frac{k_{1}^{2}-\frac{1}{4}}{\cos ^{2} \phi}+\frac{k_{2}^{2}-\frac{1}{4}}{\sin ^{2} \phi}-\frac{1}{4}\right)\right] d t\right\} \\
& =\left(\xi^{\prime} \xi^{\prime \prime} \eta^{\prime} \eta^{\prime \prime}\right)^{-1 / 2} \sum_{n=0}^{\infty} \Phi_{n}^{\left( \pm k_{2}, \pm k_{1}\right)}\left(\phi^{\prime}\right) \Phi_{n}^{\left( \pm k_{2}, \pm k_{1}\right)}\left(\phi^{\prime \prime}\right) \\
& \quad \times \int_{\mathbb{R}} \frac{d E}{2 \pi \hbar} \mathrm{e}^{-\mathrm{i} E T / \hbar} \int_{0}^{\infty} d s^{\prime \prime} \int_{\eta\left(s^{\prime \prime}\right)=\eta^{\prime \prime}} \mathcal{D} \eta(s) \int_{\xi\left(s^{\prime \prime}\right)=\xi^{\prime \prime}} \mathcal{D} \xi(s) \\
& \quad \times \exp \left\{\frac{\mathrm{i}}{\hbar} \int_{0}^{s^{\prime \prime}}\left[\frac{M}{2}\left(\left(\dot{\xi}^{2}+\dot{\eta}^{2}\right)+E\left(\xi^{2}+\eta^{2}\right)-\hbar^{2} \frac{\lambda^{2}-\frac{1}{4}}{2 M}\left(\frac{1}{\xi^{2}}+\frac{1}{\eta^{2}}\right)\right] d s\right\},\right. \\
& =\sum_{n=0}^{\infty} \int_{\mathbb{R}} d \zeta \int_{0}^{\infty} d p \mathrm{e}^{-\mathrm{i} \hbar p^{2} T / 2 M} \Psi_{n, \zeta, p}^{*}\left(\phi^{\prime}, \xi^{\prime}, \eta^{\prime}\right) \Psi_{n, \zeta, p}\left(\phi^{\prime \prime}, \xi^{\prime \prime}, \eta^{\prime \prime}\right) .
\end{aligned}
$$

In paraboloidal coordinates $\Delta V_{P F}\left(\eta_{i}\right)$ are determined by (10) and the $B\left(\eta_{i}\right)$ are given by $(i=$ $\left.1,2,3, a_{i k}=a_{i}-a_{k} a_{1}=b, a_{2}=a\right)$

$$
B\left(\eta_{i}\right)=a_{21} \frac{k_{1}^{2}-\frac{1}{4}}{\eta_{i}-a}+a_{12} \frac{k_{2}^{2}-\frac{1}{4}}{\eta_{i}-b} .
$$

The wave-functions in parabolic coordinates are given by ( $\zeta$ is the parabolic separation constant)

$$
\begin{aligned}
\Psi_{n, \zeta, p}(\phi, \xi, \eta)=\Phi_{n}^{\left( \pm k_{2}, \pm k_{1}\right)}(\phi) & \frac{\left|\Gamma\left[\frac{1+\lambda}{2}+\mathrm{i} \zeta / 2 p\right]\right|^{2}}{2 \pi \sqrt{p} \Gamma^{2}(1+\lambda)} \mathrm{e}^{\pi / 2 a p} \\
& \times M_{-\mathrm{i} \zeta / 2 p, \lambda / 2}\left(-\mathrm{i} p \xi^{2}\right) M_{-\mathrm{i} \zeta / 2 p, \lambda / 2}\left(-\mathrm{i} p \eta^{2}\right) .
\end{aligned}
$$


(ii) $k_{2}^{2}-\frac{1}{4}=0$ : We have in circular parabolic coordinates

$$
\begin{aligned}
& K^{\left(V_{1}\right)}\left(\vec{x}^{\prime \prime}, \vec{x}^{\prime} ; T\right) \\
& =\int_{\eta\left(t^{\prime}\right)=\eta^{\prime}} \int^{\left(t^{\prime \prime}\right)=\eta^{\prime \prime}} \mathcal{D} \eta(t) \int_{\xi\left(t^{\prime}\right)=\xi^{\prime}}^{\xi\left(t^{\prime \prime}\right)=\xi^{\prime \prime}} \mathcal{D} \xi(t)\left(\xi^{2}+\eta^{2}\right) \int_{z\left(t^{\prime}\right)=z^{\prime}}^{z\left(t^{\prime \prime}\right)=z^{\prime \prime}} \mathcal{D} z(t) \\
& \quad \times \exp \left\{\frac{\mathrm{i}}{\hbar} \int_{t^{\prime}}^{t^{\prime \prime}}\left[\frac{M}{2}\left(\left(\xi^{2}+\eta^{2}\right)\left(\dot{\xi}^{2}+\dot{\eta}^{2}\right)+\dot{z}^{2}\right)-\frac{\hbar^{2}}{2 M}\left(\frac{k_{1}^{2}-\frac{1}{4}}{\xi^{2} \eta^{2}}+\frac{k_{3}^{2}-\frac{1}{4}}{z^{2}}\right)\right] d t\right\} \\
& =\frac{M}{\mathrm{i} \hbar T} \exp \left[\frac{\mathrm{i} M}{2 \hbar}\left(z^{\prime 2}+z^{\prime \prime 2}\right)\right] I_{ \pm k_{3}}\left(\frac{M z^{\prime} z^{\prime \prime}}{\mathrm{i} \hbar T}\right) \\
& \quad \times \int_{\mathbb{R}} \frac{d E}{2 \pi \hbar} \mathrm{e}^{-\mathrm{i} E T / \hbar} \int_{0}^{\infty} d s^{\prime \prime} \int_{\eta\left(s^{\prime \prime}\right)=\eta^{\prime \prime}} \mathcal{D} \eta(s) \int_{\xi\left(s^{\prime \prime}\right)=\xi^{\prime \prime}} \mathcal{D} \xi(s) \\
& \quad \times \exp \left\{\frac{\mathrm{i}}{\hbar} \int_{0}^{s^{\prime \prime}}\left[\frac{M}{2}\left(\left(\dot{\xi}^{2}+\dot{\eta}^{2}\right)+E\left(\xi^{2}+\eta^{2}\right)-\hbar^{2} \frac{k_{1}^{2}-\frac{1}{4}}{2 M}\left(\frac{1}{\xi^{2}}+\frac{1}{\eta^{2}}\right)\right] d s\right\}\right. \\
& =\int_{0}^{\infty} d p_{z} \int_{\mathbb{R}} d \zeta \int_{0}^{\infty} d p \mathrm{e}^{-\mathrm{i} E T / \hbar} \Psi_{p_{z}, \zeta, p}^{*}\left(z^{\prime}, \xi^{\prime}, \eta^{\prime}\right) \Psi_{p_{z}, \zeta, p}\left(z^{\prime \prime}, \xi^{\prime \prime}, \eta^{\prime \prime}\right),
\end{aligned}
$$

with the wave-functions given by ( $\zeta$ is the parabolic separation constant)

$$
\begin{aligned}
& \Psi_{n, \zeta, p}(\phi, \xi, \eta)=\sqrt{p_{z} z} J_{ \pm k_{3}}\left(p_{z} z\right) \frac{\left|\Gamma\left[\frac{1 \pm k_{1}}{2}+\mathrm{i} \zeta / 2 p\right]\right|^{2}}{2 \pi \sqrt{p \xi \eta} \Gamma^{2}\left(1 \pm k_{1}\right)} \mathrm{e}^{\pi / 2 a p} \\
& \times M_{-\mathrm{i} \zeta / 2 p, \pm k_{1} / 2}\left(-\mathrm{i} p \xi^{2}\right) M_{-\mathrm{i} \zeta / 2 p, \pm k_{1} / 2}\left(-\mathrm{i} p \eta^{2}\right), \\
& E=\frac{\hbar^{2}}{2 M}\left(p_{z}^{2}+p^{2}\right)
\end{aligned}
$$

3.2.2. We consider the potential $\left(x_{3} \equiv z \in \mathbb{R}, k_{1,2}>0\right)$

$$
V_{2}(\vec{x})=\frac{M}{2} \omega^{2}\left(x_{1}^{2}+x_{2}^{2}+4 x_{3}^{2}\right)+\frac{\hbar^{2}}{2 M}\left(\frac{k_{1}^{2}-\frac{1}{4}}{x_{1}^{2}}+\frac{k_{2}^{2}-\frac{1}{4}}{x_{2}^{2}}\right) .
$$

We write down the corresponding path integral formulations

$$
K^{\left(V_{2}\right)}\left(\vec{x}^{\prime \prime}, \vec{x}^{\prime} ; T\right)
$$

\section{Cartesian Coordinates:}

$$
=\int_{\vec{x}\left(t^{\prime}\right)=\vec{x}^{\prime}}^{\vec{x}\left(t^{\prime \prime}\right)=\vec{x}^{\prime \prime}} \mathcal{D} \vec{x}(t) \exp \left\{\frac{\mathrm{i}}{\hbar} \int_{t^{\prime}}^{t^{\prime \prime}}\left[\frac{M}{2}\left(\dot{\vec{x}}^{2}-\omega^{2}\left(x_{1}^{2}+x_{2}^{2}+4 x_{3}^{2}\right)\right)-\frac{\hbar^{2}}{2 M}\left(\frac{k_{1}^{2}-\frac{1}{4}}{x_{1}^{2}}+\frac{k_{2}^{2}-\frac{1}{4}}{x_{2}^{2}}\right)\right] d t\right\}
$$

Parabolic Coordinates $\left(\lambda=2 n \pm k_{1} \pm k_{2}+1\right)$ :

$$
\begin{gathered}
=\int_{\eta\left(t^{\prime}\right)=\eta^{\prime}}^{\eta\left(t^{\prime \prime}\right)=\eta^{\prime \prime}} \mathcal{D} \eta(t) \int_{\xi\left(t^{\prime}\right)=\xi^{\prime}}^{\xi\left(t^{\prime \prime}\right)=\xi^{\prime \prime}} \mathcal{D} \xi(t)\left(\xi^{2}+\eta^{2}\right) \xi \eta \int_{\phi\left(t^{\prime}\right)=\phi^{\prime}}^{\phi\left(t^{\prime \prime}\right)=\phi^{\prime \prime}} \mathcal{D} \phi(t) \\
\quad \times \exp \left\{\frac { \mathrm { i } } { \hbar } \int _ { t ^ { \prime } } ^ { t ^ { \prime \prime } } \left[\frac{M}{2}\left(\left(\xi^{2}+\eta^{2}\right)\left(\dot{\xi}^{2}+\dot{\eta}^{2}\right)+\xi^{2} \eta^{2} \dot{\phi}^{2}-\omega^{2}\left(\xi^{2} \eta^{2}+\left(\xi^{2}-\eta^{2}\right)^{2}\right)\right)\right.\right. \\
\left.\left.-\frac{\hbar^{2}}{2 M \xi^{2} \eta^{2}}\left(\frac{k_{1}^{2}-\frac{1}{4}}{\cos ^{2} \phi}+\frac{k_{2}^{2}-\frac{1}{4}}{\sin ^{2} \phi}-\frac{1}{4}\right)\right] d t\right\}
\end{gathered}
$$




$$
\begin{aligned}
& =\left(\xi^{\prime} \xi^{\prime \prime} \eta^{\prime} \eta^{\prime \prime}\right)^{-1 / 2} \sum_{n=0}^{\infty} \Phi_{n}^{\left( \pm k_{2}, \pm k_{1}\right)}\left(\phi^{\prime}\right) \Phi_{n}^{\left( \pm k_{2}, \pm k_{1}\right)}\left(\phi^{\prime \prime}\right) \\
& \quad \times \int_{\mathbb{R}} \frac{d E}{2 \pi \hbar} \mathrm{e}^{-\mathrm{i} E T / \hbar} \int_{0}^{\infty} d s^{\prime \prime} \int_{\eta(0)=\eta^{\prime}}^{\xi\left(s^{\prime \prime}\right)=\eta^{\prime \prime}} \mathcal{D} \eta(s) \int_{\xi(0)=\xi^{\prime}}^{\xi\left(s^{\prime \prime}\right)=\xi^{\prime \prime}} \mathcal{D} \xi(s) \\
& \quad \times \exp \left\{\frac{\mathrm{i}}{\hbar} \int_{0}^{s^{\prime \prime}}\left[\frac{M}{2}\left(\left(\dot{\xi}^{2}+\dot{\eta}^{2}\right)-\omega^{2}\left(\xi^{6}+\eta^{6}\right)\right)+E\left(\xi^{2}+\eta^{2}\right)-\hbar^{2} \frac{\lambda^{2}-\frac{1}{4}}{2 M}\left(\frac{1}{\xi^{2}}+\frac{1}{\eta^{2}}\right)\right] d s\right\}
\end{aligned}
$$

Circular Polar Coordinates:

$$
\begin{aligned}
& =\int_{\rho\left(t^{\prime}\right)=\rho^{\prime}}^{\rho\left(t^{\prime \prime}\right)=\rho^{\prime \prime}} \rho \mathcal{D} \rho(t) \int_{\phi\left(t^{\prime}\right)=\phi^{\prime}}^{\phi\left(t^{\prime \prime}\right)=\phi^{\prime \prime}} \mathcal{D} \phi(t) \int_{z\left(t^{\prime}\right)=z^{\prime}}^{z\left(t^{\prime \prime}\right)=z^{\prime \prime}} \mathcal{D} z(t) \\
& \quad \times \exp \left\{\frac{\mathrm{i}}{\hbar} \int_{t^{\prime}}^{t^{\prime \prime}}\left[\frac{M}{2}\left(\dot{\rho}^{2}+\rho^{2} \dot{\phi}^{2}+\dot{z}^{2}-\omega^{2}\left(\rho^{2}+4 z^{2}\right)\right)-\frac{\hbar^{2}}{2 M \rho^{2}}\left(\frac{k_{1}^{2}-\frac{1}{4}}{\cos ^{2} \phi}+\frac{k_{2}^{2}-\frac{1}{4}}{\sin ^{2} \phi}-\frac{1}{4}\right)\right] d t\right\}
\end{aligned}
$$

Circular Elliptic Coordinates:

$$
\begin{gathered}
=\int_{\xi\left(t^{\prime}\right)=\xi^{\prime}} \mathcal{D} \xi(t) \int_{\eta\left(t^{\prime}\right)=\eta^{\prime}}^{\eta\left(t^{\prime \prime}\right)=\eta^{\prime \prime}} \mathcal{D} \eta(t) d^{2}\left(\sinh ^{2} \xi+\sin ^{2} \eta\right) \int_{z\left(t^{\prime}\right)=z^{\prime}}^{z\left(t^{\prime \prime}\right)=z^{\prime \prime}} \mathcal{D} z(t) \\
\quad \times \exp \left\{\frac { \mathrm { i } } { \hbar } \int _ { t ^ { \prime } } ^ { t ^ { \prime \prime } } \left[\frac{M}{2} d^{2}\left(\left(\sinh ^{2} \xi+\sin ^{2} \eta\right)\left(\dot{\xi}^{2}+\dot{\eta}^{2}\right)-\omega^{2}\left(\cosh ^{2} \xi \cos ^{2} \eta+\sinh ^{2} \xi \sin ^{2} \eta\right)+4 z^{2}\right)\right.\right. \\
\left.\left.-\frac{\hbar^{2}}{2 M d^{2}}\left(\frac{k_{1}^{2}-\frac{1}{4}}{\cosh ^{2} \xi \cos ^{2} \eta}+\frac{k_{2}^{2}-\frac{1}{4}}{\sinh ^{2} \xi \sin ^{2} \eta}\right)\right] d t\right\} .
\end{gathered}
$$

and $\Phi_{n}^{(\alpha, \beta)}$ denote the wave-functions (88). $K^{\left(V_{2}\right)}(T)$ is explicitly soluble in cartesian and circular polar coordinates by means of the harmonic oscillator in $z$, and the radial harmonic oscillator in $x_{1}, x_{2}$, respectively (note the similarity to the Holt potential)

$$
K^{\left(V_{2}\right)}\left(\vec{x}^{\prime \prime}, \vec{x}^{\prime} ; T\right)
$$

\section{Cartesian Coordinates:}

$$
\begin{aligned}
& =\sqrt{\frac{M \omega}{\mathrm{i} \hbar \sin 2 \omega T}} \exp \left\{-\frac{M \omega}{\mathrm{i} \hbar \sin 2 \omega T}\left[\left(z^{\prime 2}+z^{\prime \prime 2}\right) \cos 2 \omega T-2 z^{\prime} z^{\prime \prime}\right]\right\} \\
& \times\left(\frac{M \omega}{\mathrm{i} \hbar \sin \omega T}\right)^{2} \prod_{i=1}^{2} \sqrt{x_{i}^{\prime} x_{i}^{\prime \prime}} \exp \left[-\frac{M \omega}{2 \mathrm{i} \hbar}\left(x_{i}^{\prime 2}+x_{i}^{\prime \prime 2}\right) \cot \omega T\right] I_{k_{i}}\left(\frac{M \omega x_{i}^{\prime} x_{i}^{\prime \prime}}{\mathrm{i} \hbar \sin \omega T}\right) \\
& =\sum_{n_{z}, n_{1}, n_{2}=0}^{\infty} \mathrm{e}^{-\mathrm{i} E_{N} T / \hbar} \Psi_{n_{z}, n_{1}, n_{2}}\left(z^{\prime}, x_{1}^{\prime}, x_{2}^{\prime}\right) \Psi_{n_{z}, n_{1}, n_{2}}\left(z^{\prime \prime}, x_{1}^{\prime \prime}, x_{2}^{\prime \prime}\right)
\end{aligned}
$$

Circular Polar Coordinates $\left(\lambda=2 n \pm k_{1} \pm k_{2}+1\right)$ :

$$
\begin{aligned}
= & \sqrt{\frac{M \omega}{\mathrm{i} \hbar \sin 2 \omega T}} \exp \left\{-\frac{M \omega}{\mathrm{i} \hbar \sin 2 \omega T}\left[\left(z^{\prime 2}+z^{\prime \prime 2}\right) \cos 2 \omega T-2 z^{\prime} z^{\prime \prime}\right]\right\} \\
& \times \frac{M \omega}{\mathrm{i} \hbar \sin \omega T} \sum_{n=0}^{\infty} \Phi_{n}^{\left( \pm k_{2}, \pm k_{1}\right)}\left(\phi^{\prime}\right) \Phi_{n}^{\left( \pm k_{2}, \pm k_{1}\right)}\left(\phi^{\prime \prime}\right) \exp \left[-\frac{M \omega}{2 \mathrm{i} \hbar}\left(\rho^{\prime 2}+\rho^{\prime \prime 2}\right) \cot \omega T\right] I_{\lambda}\left(\frac{M \omega \rho^{\prime} \rho^{\prime \prime}}{\mathrm{i} \hbar \sin \omega T}\right)
\end{aligned}
$$




$$
=\sum_{n_{z}, n, m=0}^{\infty} \mathrm{e}^{-\mathrm{i} E_{N} T / \hbar} \Psi_{n_{z}, n, m}\left(z^{\prime}, \phi^{\prime}, \rho^{\prime}\right) \Psi_{n_{z}, n, m}\left(z^{\prime \prime}, \phi^{\prime \prime}, \rho^{\prime \prime}\right),
$$

where the wave-functions and the energy-levels are given by ( $N$ is the principal quantum number)

Cartesian Coordinates:

$$
\begin{aligned}
& \Psi_{n_{z}, n_{1}, n_{2}}\left(x_{1}, x_{2}, z\right)=\frac{2 M \omega}{\hbar} \prod_{i=1}^{2}\left(\frac{M \omega}{\hbar}\right)^{ \pm k_{i} / 2} \sqrt{\frac{n_{i} !}{\Gamma\left(n_{i} \pm k_{i}+1\right)}} \\
& \times x_{i}^{1 / 2 \pm k_{i}} \exp \left(-\frac{M \omega}{2 \hbar} x_{i}^{2}\right) L_{n_{i}}^{\left( \pm k_{i}\right)}\left(\frac{M \omega}{\hbar} x_{i}^{2}\right) \\
& \times \sqrt{\sqrt{\frac{2 M \omega}{\pi \hbar}} \frac{1}{2^{n_{z}} n_{z} !}} \exp \left(-\frac{M \omega}{\hbar} z^{2}\right) H_{n_{z}}\left(\sqrt{\left.\frac{2 M \omega}{\hbar} z\right),}\right. \\
& E_{N}=\hbar \omega\left.N \pm k_{1} \pm k_{2}+\frac{5}{2}\right), \quad N=2\left(n_{1}+n_{2}\right)+n_{z},
\end{aligned}
$$

Circular Polar Coordinates $\left(\lambda=2 n \pm k_{1} \pm k_{2}+1\right)$ :

$$
\begin{gathered}
\Psi_{n_{z}, n, m}(z, \phi, \rho)=\Phi_{n}^{\left( \pm k_{2}, \pm k_{1}\right)}(\phi) \sqrt{\sqrt{\frac{2 M \omega}{\pi \hbar}} \frac{1}{2^{n_{z}} n_{z} !}} \exp \left(-\frac{M \omega}{\hbar} z^{2}\right) H_{n_{z}}\left(\sqrt{\left.\frac{2 M \omega}{\hbar} z\right)}\right. \\
\times \sqrt{\frac{2 M \omega}{\hbar}}\left(\frac{M \omega}{\hbar} \rho^{2}\right)^{\lambda / 2} \sqrt{\frac{m !}{\Gamma(m+\lambda+1)}} \exp \left(-\frac{M \omega}{2 \hbar} \rho^{2}\right) L_{m}^{(\lambda)}\left(\frac{M \omega}{\hbar} \rho^{2}\right), \\
E_{N}=\hbar \omega\left(N \pm k_{1} \pm k_{2}+\frac{5}{2}\right), \quad N=2(n+m)+n_{z},
\end{gathered}
$$

and the $\Phi_{n}^{\left( \pm k_{2}, \pm k_{1}\right)}(\phi)$ denote the wave-functions (88).

3.2.3. We consider the three-dimensional potential $\left(k_{1,2}>0\right)$

$$
V_{3}(\vec{x})=-\frac{\alpha}{\sqrt{x^{2}+y^{2}+z^{2}}}+\frac{\hbar^{2}}{2 M}\left(\frac{k_{1}^{2}-\frac{1}{4}}{x^{2}}+\frac{k_{2}^{2}-\frac{1}{4}}{y^{2}}\right) .
$$

This potential can be interpreted as a maximally super-integrable generalization of the Coulomb potential. Note furthermore that the pure Coulomb potential has the same separating coordinate systems as $V_{3}(\vec{x})$ and therefore the additional centrifugal terms do not spoil this property. The pure Coulomb path integral was discussed by many authors, e.g. Castrigiano and Stärk [15], Chetouani and Hammann [19, 20], Duru and Grosche [56], Kleinert [30, 31], Inomata [69], Kleinert [84], Pak and Sökmen [109], Steiner [118], and Storchak [122]. We formulate the path integrals in which this potential is separable

$$
\begin{aligned}
& K^{\left(V_{3}\right)}\left(\vec{x}^{\prime \prime}, \vec{x}^{\prime} ; T\right) \\
& =\int_{\vec{x}\left(t^{\prime \prime}\right)=\vec{x}^{\prime \prime}} \mathcal{D} \vec{x}(t) \exp \left\{\frac{\mathrm{i}}{\hbar} \int_{t^{\prime}}^{t^{\prime \prime}}\left[\frac{M}{2} \dot{\vec{x}}^{2}+\frac{\alpha}{r}-\frac{\hbar^{2}}{2 M} \sum_{i=1}^{2} \frac{k_{i}^{2}-\frac{1}{4}}{x_{i}^{2}}\right] d t\right\}
\end{aligned}
$$

Conical Coordinates:

$$
\begin{aligned}
& =\int_{r\left(t^{\prime}\right)=r^{\prime}}^{r\left(t^{\prime \prime}\right)=r^{\prime \prime}} r^{2} \mathcal{D} r(t) \int_{\alpha\left(t^{\prime}\right)=\alpha^{\prime}}^{\alpha\left(t^{\prime \prime}\right)=\alpha^{\prime \prime}} \mathcal{D} \alpha(t) \int_{\beta\left(t^{\prime}\right)=\beta^{\prime}}^{\beta\left(t^{\prime \prime}\right)=\beta^{\prime \prime}} \mathcal{D} \beta(t)\left(k^{2} \mathrm{cn}^{2} \alpha+{k^{\prime}}^{2} \mathrm{cn}^{2} \beta\right) \\
& \quad \times \exp \left\{\frac { \mathrm { i } } { \hbar } \int _ { t ^ { \prime } } ^ { t ^ { \prime \prime } } \left[\frac{M}{2}\left(\dot{r}^{2}+r^{2}\left(k^{2} \mathrm{cn}^{2} \alpha+k^{\prime 2} \mathrm{cn}^{2} \beta\right)\left(\dot{\alpha}^{2}+\dot{\beta}^{2}\right)\right)+\frac{\alpha}{r}\right.\right.
\end{aligned}
$$




$$
\left.\left.-\frac{\hbar^{2}}{2 M}\left(\frac{k_{1}^{2}-\frac{1}{4}}{\operatorname{sn}^{2} \alpha \operatorname{dn}^{2} \beta}+\frac{k_{2}^{2}-\frac{1}{4}}{\operatorname{cn}^{2} \alpha \operatorname{cn}^{2} \beta}\right)\right] d t\right\}
$$

Spherical Coordinates:

$$
\begin{aligned}
& =\int_{r\left(t^{\prime}\right)=r^{\prime}}^{r\left(t^{\prime \prime}\right)=r^{\prime \prime}} r^{2} \mathcal{D} r(t) \int_{\theta\left(t^{\prime}\right)=\theta^{\prime}}^{\theta\left(t^{\prime \prime}\right)=\theta^{\prime \prime}} \sin \theta \mathcal{D} \theta(t) \int_{\phi\left(t^{\prime}\right)=\phi^{\prime}}^{\phi\left(t^{\prime \prime}\right)=\phi^{\prime \prime}} \mathcal{D} \phi(t) \\
& \quad \times \exp \left\{\frac { \mathrm { i } } { \hbar } \int _ { t ^ { \prime } } ^ { t ^ { \prime \prime } } \left[\frac{M}{2}\left(\dot{r}^{2}+r^{2} \dot{\theta}^{2}+r^{2} \sin ^{2} \theta \dot{\phi}^{2}\right)\right.\right. \\
& \left.\left.+\frac{\alpha}{r}-\frac{\hbar^{2}}{2 M r^{2} \sin ^{2} \theta}\left(\frac{k_{1}^{2}-\frac{1}{4}}{\cos ^{2} \phi}+\frac{k_{2}^{2}-\frac{1}{4}}{\sin ^{2} \phi}-\frac{1}{4}\right)\right] d t\right\}
\end{aligned}
$$

Parabolic Coordinates:

$$
\begin{aligned}
& =\int_{\eta\left(t^{\prime}\right)=\eta^{\prime}}^{\eta\left(t^{\prime \prime}\right)=\eta^{\prime \prime}} \mathcal{D} \eta(t) \int_{\xi\left(t^{\prime}\right)=\xi^{\prime}}^{\xi\left(t^{\prime \prime}\right)=\xi^{\prime \prime}} \mathcal{D} \xi(t)\left(\xi^{2}+\eta^{2}\right) \xi \eta \int_{\phi\left(t^{\prime}\right)=\phi^{\prime}}^{\phi\left(t^{\prime \prime}\right)=\phi^{\prime \prime}} \mathcal{D} \phi(t) \\
& \times \exp \left\{\frac { \mathrm { i } } { \hbar } \int _ { t ^ { \prime } } ^ { t ^ { \prime \prime } } \left[\frac{M}{2}\left(\left(\xi^{2}+\eta^{2}\right)\left(\dot{\xi}^{2}+\dot{\eta}^{2}\right)+\xi^{2} \eta^{2} \dot{\phi}^{2}\right)\right.\right. \\
& \left.\left.\quad+\frac{2 \alpha}{\xi^{2}+\eta^{2}}-\frac{\hbar^{2}}{2 M \xi^{2} \eta^{2}}\left(\frac{k_{1}^{2}-\frac{1}{4}}{\cos ^{2} \phi}+\frac{k_{2}^{2}-\frac{1}{4}}{\sin ^{2} \phi}-\frac{1}{4}\right)\right] d t\right\}
\end{aligned}
$$

Prolate Spheroidal II Coordinates $\left(\lambda_{1}=2 n \pm k_{1} \pm k_{2}+1\right)$ :

$$
\begin{gathered}
=\int_{\mu\left(t^{\prime}\right)=\mu^{\prime}}^{\mu\left(t^{\prime \prime}\right)=\mu^{\prime \prime}} \mathcal{D} \mu(t) \int_{\nu\left(t^{\prime}\right)=\nu^{\prime}}^{\nu\left(t^{\prime \prime}\right)=\nu^{\prime \prime}} \mathcal{D} \nu(t) d^{3}\left(\sinh ^{2} \mu+\sin ^{2} \nu\right) \sin \nu \sinh \mu \int_{\phi\left(t^{\prime}\right)=\phi^{\prime}}^{\phi\left(t^{\prime \prime}\right)=\phi^{\prime \prime}} \mathcal{D} \phi(t) \\
\times \exp \left\{\frac { \mathrm { i } } { \hbar } \int _ { t ^ { \prime } } ^ { t ^ { \prime \prime } } \left[\frac{M}{2} d^{2}\left(\left(\sinh ^{2} \mu+\sin ^{2} \nu\right)\left(\dot{\mu}^{2}+\dot{\nu}^{2}\right)+\sinh ^{2} \mu \sin ^{2} \nu \dot{\phi}^{2}\right)\right.\right. \\
\left.\left.+\frac{\alpha}{d(\cosh \mu+\cos \nu)}-\frac{\hbar^{2}}{2 M d^{2} \sinh ^{2} \mu \sin ^{2} \nu}\left(\frac{k_{1}^{2}-\frac{1}{4}}{\cos ^{2} \phi}+\frac{k_{2}^{2}-\frac{1}{4}}{\sin ^{2} \phi}-\frac{1}{4}\right)\right] d t\right\} \\
=\left(d^{2} \sinh \mu^{\prime} \sinh \mu^{\prime \prime} \sin \nu^{\prime} \sin \nu^{\prime \prime}\right)^{-1 / 2} \sum_{n=0}^{\infty} \Phi_{n}^{\left( \pm k_{2}, \pm k_{1}\right)}\left(\phi^{\prime \prime}\right) \Phi_{n}^{\left( \pm k_{2}, \pm k_{1}\right)}\left(\phi^{\prime}\right) \int_{\mathbb{R}} \frac{d E}{2 \pi \hbar} \mathrm{e}^{-\mathrm{i} E T / \hbar \int_{0}^{\infty}} d s^{\prime \prime} \\
\quad \int_{\mu\left(s^{\prime \prime}\right)=\mu^{\prime \prime} \quad} \quad \int_{\nu\left(s^{\prime \prime}\right)=\nu^{\prime \prime}} \mathcal{D} \nu(s) \exp \left\{\frac { \mathrm { i } } { \hbar } \int _ { 0 } ^ { s ^ { \prime \prime } } \left[\frac{M}{2}\left(\dot{\mu}^{2}+\dot{\nu}^{2}\right)+E d^{2}\left(\cosh ^{2} \mu-\cos ^{2} \nu\right)\right.\right. \\
\quad \int_{\nu(0)=\nu^{\prime}}(203) \\
\left.\left.+\alpha d(\cosh \mu-\cos \nu)-\frac{\hbar^{2}}{2 M}\left(\frac{\lambda_{1}^{2}-\frac{1}{4}}{\sinh ^{2} \mu}+\frac{\lambda_{1}^{2}-\frac{1}{4}}{\sin ^{2} \nu}\right)\right] d s\right\} .
\end{gathered}
$$

We now obtain $\left(\lambda_{1}=2 n \pm k_{1} \pm k_{2}+1, \lambda_{2}=m+\lambda_{1}+\frac{1}{2}, \kappa=\alpha \sqrt{-M / 2 E} / \hbar\right.$, only the radial Green function can be explicitly evaluated)

$\frac{\mathrm{i}}{\hbar} \int_{0}^{\infty} d T \mathrm{e}^{\mathrm{i} T E / \hbar} K^{\left(V_{3}\right)}\left(\vec{x}^{\prime \prime}, \vec{x}^{\prime} ; T\right)$

Spherical Coordinates: 


$$
\begin{aligned}
= & \sum_{n=0}^{\infty} \Phi_{n}^{\left( \pm k_{2}, \pm k_{1}\right)}\left(\phi^{\prime \prime}\right) \Phi_{n}^{\left( \pm k_{2}, \pm k_{1}\right)}\left(\phi^{\prime}\right) \sum_{m=0}^{\infty}\left(m+\lambda_{1}+\frac{1}{2}\right) \frac{\Gamma\left(m+\lambda_{1}+1\right)}{m !} P_{\lambda_{1}+m}^{-\lambda_{1}}\left(\cos \theta^{\prime \prime}\right) P_{\lambda_{1}+m}^{-\lambda_{1}}\left(\cos \theta^{\prime}\right) \\
& \times \frac{1}{r^{\prime} r^{\prime \prime}} \frac{1}{\hbar} \sqrt{-\frac{M}{2 E}} \frac{\Gamma\left(\frac{1}{2}+\lambda_{2}-\kappa\right)}{\Gamma\left(2 \lambda_{2}+1\right)} W_{\kappa, \lambda_{2}}\left(\sqrt{-8 M E} \frac{r_{>}}{\hbar}\right) M_{\kappa, \lambda_{2}}\left(\sqrt{-8 M E} \frac{r_{<}}{\hbar}\right) \\
= & \sum_{n, m=0}^{\infty}\left\{\sum_{l=0}^{\infty} \frac{\Psi_{n, m, l}^{*}\left(\theta^{\prime}, \phi^{\prime}, r^{\prime}\right) \Psi_{n, m, l}\left(\theta^{\prime \prime}, \phi^{\prime \prime}, r^{\prime \prime}\right)}{E_{N}-E}+\int_{\mathbb{R}} d p \frac{\Psi_{n, m, p}^{*}\left(\theta^{\prime}, \phi^{\prime}, r^{\prime}\right) \Psi_{n, m, p}^{\alpha)}\left(\theta^{\prime \prime}, \phi^{\prime \prime}, r^{\prime \prime}\right)}{\hbar^{2} p^{2} / 2 M-E}\right\}
\end{aligned}
$$

Parabolic Coordinates $(\omega=\sqrt{-2 E / M})$ :

$$
\begin{aligned}
=\left(\frac{M}{\mathrm{i} \hbar}\right)^{2} \sum_{n=0}^{\infty} \Phi_{n}^{\left( \pm k_{2}, \pm k_{1}\right)}\left(\phi^{\prime \prime}\right) \Phi_{n}^{\left( \pm k_{2}, \pm k_{1}\right)}\left(\phi^{\prime}\right) \int_{0}^{\infty} \frac{\omega^{2} d s^{\prime \prime}}{\sin ^{2} \omega s^{\prime \prime}} \mathrm{e}^{2 \mathrm{i} \alpha s^{\prime \prime} / \hbar} \\
\quad \times I_{\lambda_{1}}\left(\frac{M \omega \eta^{\prime} \eta^{\prime \prime}}{\mathrm{i} \hbar \sin \omega s^{\prime \prime}}\right) I_{\lambda_{1}}\left(\frac{M \omega \xi^{\prime} \xi^{\prime \prime}}{\mathrm{i} \hbar \sin \omega s^{\prime \prime}}\right) \exp \left[-\frac{M \omega}{2 \mathrm{i} \hbar}\left(\xi^{\prime 2}+\xi^{\prime \prime 2}+\eta^{\prime 2}+\eta^{\prime \prime 2}\right) \cot \omega s^{\prime \prime}\right] \\
=\sum_{n=0}^{\infty}\left[\sum_{n_{1}, n_{2}=0}^{\infty} \frac{\Psi_{n, n_{1}, n_{2}}\left(\phi^{\prime}, \xi^{\prime}, \eta^{\prime}\right) \Psi_{n, n_{1}, n_{2}}\left(\phi^{\prime \prime}, \xi^{\prime \prime}, \eta^{\prime \prime}\right)}{E_{N}-E}\right. \\
\left.+\int_{0}^{\infty} d p \int_{\mathbb{R}} d \zeta \frac{\Psi_{n, p, \zeta}^{*}\left(\phi^{\prime}, \xi^{\prime}, \eta^{\prime}\right) \Psi_{n, p, \zeta}\left(\phi^{\prime \prime}, \xi^{\prime \prime}, \eta^{\prime \prime}\right)}{\hbar^{2} p^{2} / 2 M-E}\right] .
\end{aligned}
$$

The discrete state wave-functions in the spherical coordinates are

$$
\begin{aligned}
\Psi_{n, m, l}(\theta, \phi, r)= & \Phi_{n}^{\left( \pm k_{2}, \pm k_{1}\right)}(\phi) \sqrt{\left(m+\lambda_{1}+\frac{1}{2}\right) \frac{\Gamma\left(m+\lambda_{1}+1\right)}{m !}} P_{\lambda_{1}+m}^{-\lambda_{1}}(\cos \theta) \\
& \times \frac{2}{\left(n+\lambda_{1}+\frac{1}{2}\right)^{2}}\left[\frac{2 l !}{a^{3}\left(l+\lambda_{2}+\frac{1}{2}\right) \Gamma\left(l+2 \lambda_{2}+1\right)}\right]^{1 / 2}\left(\frac{2 r}{a\left(l+\lambda_{2}+\frac{1}{2}\right)}\right)^{\lambda_{2}} \\
& \times \exp \left(-\frac{r}{a\left(l+\lambda_{2}+\frac{1}{2}\right)}\right) L_{l}^{\left(2 \lambda_{2}\right)}\left(\frac{2 r}{a\left(l+\lambda_{2}+\frac{1}{2}\right)}\right) \\
E_{N}= & -\frac{M \alpha^{2}}{\hbar^{2} N^{2}}, \quad N=l+m+2 n \pm k_{1} \pm k_{2}+1
\end{aligned}
$$

The continuous wave-functions are

$$
\begin{gathered}
\Psi_{n, m, p}(\theta, \phi, r)=\Phi_{n}^{\left( \pm k_{2}, \pm k_{1}\right)}(\phi) \sqrt{\left(m+\lambda_{1}+\frac{1}{2}\right) \frac{\Gamma\left(m+\lambda_{1}+1\right)}{m !}} P_{\lambda_{1}+m}^{-\lambda_{1}}(\cos \theta) \\
\times \frac{\Gamma\left(\frac{1}{2}+\lambda_{2}-\mathrm{i} / a p\right)}{\sqrt{2 \pi} r \Gamma\left(2 \lambda_{2}+1\right)} \exp \left(\frac{\pi}{2 a p}\right) M_{\mathrm{i} / a p, \lambda_{2}}(-2 \mathrm{i} p r) .
\end{gathered}
$$

The discrete state wave-functions in parabolic coordinates are

$$
\begin{aligned}
\Psi_{n, n_{1}, n_{2}}(\phi, \xi, \eta)= & \Phi_{n}^{\left( \pm k_{2}, \pm k_{1}\right)}(\phi)\left[\frac{2}{a^{2} N^{3}} \cdot \frac{2 n_{1} ! n_{2} !}{\Gamma\left(n_{1}+\lambda_{1}+1\right) \Gamma\left(n_{2}+\lambda_{1}+1\right)}\right]^{1 / 2} \\
& \times\left(\frac{\xi \eta}{(a N)^{2}}\right)^{\lambda_{1}} \exp \left(-\frac{\xi^{2}+\eta^{2}}{2 a N}\right) L_{n_{1}}^{\left(\lambda_{1}\right)}\left(\frac{\xi^{2}}{a N}\right) L_{n_{2}}^{\left(\lambda_{1}\right)}\left(\frac{\eta^{2}}{a N}\right), \\
E_{N}= & -\frac{M \alpha^{2}}{\hbar^{2} N^{2}}, \quad N=n_{1}+n_{2}+2 n \pm k_{1} \pm k_{2}+1 .
\end{aligned}
$$

The continuous wave-functions are

$$
\begin{gathered}
\Psi_{n, p, \zeta}(\phi, \xi, \eta)=\Phi_{n}^{\left( \pm k_{2}, \pm k_{1}\right)}(\phi) \frac{\Gamma\left[\frac{1+\lambda_{1}}{2}+\frac{\mathrm{i}}{2 p}(1 / a+\zeta)\right] \Gamma\left[\frac{1+\lambda_{1}}{2}+\frac{\mathrm{i}}{2 p}(1 / a-\zeta)\right]}{2 \pi \sqrt{p} \xi \eta \Gamma\left(1+\lambda_{1}\right) \Gamma\left(1+\lambda_{1}\right)} \mathrm{e}^{\pi / 2 a p} \\
\times M_{-\mathrm{i}(1 / a+\zeta) / 2 p, \lambda_{1} / 2}\left(-\mathrm{i} p \xi^{2}\right) M_{-\mathrm{i}(1 / a-\zeta) / 2 p, \lambda_{1} / 2}\left(-\mathrm{i} p \eta^{2}\right) .
\end{gathered}
$$


For the expansion into the wave-functions one uses (135) and performs the variable substitution $\left(p_{\xi}, p_{\eta}\right) \mapsto[(1 / a+\zeta) / 2 p,(1 / a-\zeta) / 2 p], a=\hbar^{2} / M \alpha . \zeta$ is the parabolic separation constant.

3.2.4. We consider the potential $\left(k_{1,2,3}>0\right)$

$$
V_{4}(\vec{x})=\frac{\hbar^{2}}{2 M}\left(\frac{k_{1}^{2} x}{y^{2} \sqrt{x^{2}+y^{2}}}+\frac{k_{2}^{2}-\frac{1}{4}}{y^{2}}+\frac{k_{3}^{2}-\frac{1}{4}}{z^{2}}\right) .
$$

Note that for $k_{3}^{2}-\frac{1}{4}=0$ we obtain a trivial extension of the $\alpha=0$ case of the two-dimensional maximally super-integrable potential $V_{3}(\vec{x})$. We formulate the path integral in spherical, circular polar, circular parabolic and circular elliptic II coordinates, respectively

$$
\begin{aligned}
& K^{\left(V_{4}\right)}\left(\vec{x}^{\prime \prime}, \vec{x}^{\prime} ; T\right) \\
& =\int_{\vec{x}\left(t^{\prime}\right)=\vec{x}^{\prime}}^{\vec{x}\left(t^{\prime \prime}\right)=\vec{x}^{\prime \prime}} \mathcal{D} \vec{x}(t) \exp \left\{\frac{\mathrm{i}}{\hbar} \int_{t^{\prime}}^{t^{\prime \prime}}\left[\frac{M}{2} \dot{\vec{x}}^{2}-\frac{\hbar^{2}}{2 M}\left(\frac{k_{1}^{2} x}{y^{2} \sqrt{x^{2}+y^{2}}}+\frac{k_{2}^{2}-\frac{1}{4}}{y^{2}}+\frac{k_{3}^{2}-\frac{1}{4}}{z^{2}}\right)\right] d t\right\}
\end{aligned}
$$

Spherical Coordinates:

$$
\begin{aligned}
& =\int_{r\left(t^{\prime}\right)=r^{\prime}}^{r\left(t^{\prime \prime}\right)=r^{\prime \prime}} r^{2} \mathcal{D} r(t) \int_{\theta\left(t^{\prime}\right)=\theta^{\prime}}^{\theta\left(t^{\prime \prime}\right)=\theta^{\prime \prime}} \sin \theta \mathcal{D} \theta(t) \int_{\phi\left(t^{\prime}\right)=\phi^{\prime}}^{\phi\left(t^{\prime \prime}\right)=\phi^{\prime \prime}} \mathcal{D} \phi(t) \\
& \quad \times \exp \left\{\frac { \mathrm { i } } { \hbar } \int _ { t ^ { \prime } } ^ { t ^ { \prime \prime } } \left[\frac{M}{2}\left(\dot{r}^{2}+r^{2} \dot{\theta}^{2}+r^{2} \sin ^{2} \theta \dot{\phi}^{2}\right)\right.\right. \\
& \left.\left.\quad-\frac{\hbar^{2}}{2 M r^{2}}\left(\frac{1}{\sin ^{2} \theta}\left(\frac{k_{2}^{2}+k_{1}^{2}-\frac{1}{4}}{4 \sin ^{2} \frac{\phi}{2}}+\frac{k_{2}^{2}-k_{1}^{2}-\frac{1}{4}}{4 \cos ^{2} \frac{\phi}{2}}-\frac{1}{4}\right)+\frac{k_{3}^{2}-\frac{1}{4}}{\cos ^{2} \theta}-\frac{1}{4}\right)\right] d t\right\}
\end{aligned}
$$

Circular Polar Coordinates:

$$
\begin{aligned}
& =\int_{\rho\left(t^{\prime}\right)=\rho^{\prime}}^{\rho\left(t^{\prime \prime}\right)=\rho^{\prime \prime}} \rho \mathcal{D} \rho(t) \int_{\phi\left(t^{\prime}\right)=\phi^{\prime}}^{\phi\left(t^{\prime \prime}\right)=\phi^{\prime \prime}} \mathcal{D} \phi(t) \int_{z\left(t^{\prime}\right)=z^{\prime}}^{z\left(t^{\prime \prime}\right)=z^{\prime \prime}} \mathcal{D} z(t) \\
& \quad \times \exp \left(\frac { \mathrm { i } } { \hbar } \int _ { t ^ { \prime } } ^ { t ^ { \prime \prime } } \left\{\frac{M}{2}\left(\dot{\rho}^{2}+\rho^{2} \dot{\phi}^{2}+\dot{z}^{2}\right)\right.\right. \\
& \left.\left.\quad-\frac{\hbar^{2}}{2 M}\left[\frac{1}{\rho^{2}}\left(\frac{k_{2}^{2}-k_{1}^{2}-\frac{1}{4}}{4 \cos ^{2} \frac{\phi}{2}}+\frac{k_{2}^{2}+k_{1}^{2}-\frac{1}{4}}{4 \sin ^{2} \frac{\phi}{2}}-\frac{1}{4}\right)+\frac{k_{3}^{2}-\frac{1}{4}}{z^{2}}\right]\right\} d t\right)
\end{aligned}
$$

Circular Elliptic II Coordinates:

$$
\begin{aligned}
& =\int_{\xi\left(t^{\prime}\right)=\xi^{\prime}}^{\xi\left(t^{\prime \prime}\right)=\xi^{\prime \prime}} \mathcal{D} \xi(t) \int_{\eta\left(t^{\prime}\right)=\eta^{\prime}}^{\eta\left(t^{\prime \prime}\right)=\eta^{\prime \prime}} \mathcal{D} \eta(t) d^{2}\left(\sinh ^{2} \xi+\sin ^{2} \eta\right) \int_{z\left(t^{\prime}\right)=z^{\prime}}^{z\left(t^{\prime \prime}\right)=z^{\prime \prime}} \mathcal{D} z(t) \\
& \times \exp \left\{\frac { \mathrm { i } } { \hbar } \int _ { t ^ { \prime } } ^ { t ^ { \prime \prime } } \left[\frac{M}{2}\left(\left(\sinh ^{2} \xi^{2}+\sin ^{2} \eta\right)\left(\dot{\xi}^{2}+\dot{\eta}^{2}\right)+\dot{z}^{2}\right)+\frac{\alpha}{d(\cosh \xi+\cos \eta)}\right.\right. \\
& \left.\left.-\frac{\hbar^{2}}{2 M}\left(\frac{1}{d^{2} \sinh ^{2} \xi \sin ^{2} \eta}\left(k_{1}^{2} \frac{\cosh \xi \cos \eta+1}{\cosh \xi+\cos \eta}+k_{2}^{2}-\frac{1}{2}\right)+\frac{k_{3}^{2}-\frac{1}{4}}{z^{2}}\right)\right] d t\right\}
\end{aligned}
$$

Circular Parabolic Coordinates:

$$
\begin{aligned}
& =\int_{\eta\left(t^{\prime}\right)=\eta^{\prime}}^{\eta\left(t^{\prime \prime}\right)=\eta^{\prime \prime}} \mathcal{D} \eta(t) \int_{\xi\left(t^{\prime}\right)=\xi^{\prime}}^{\xi\left(t^{\prime \prime}\right)=\xi^{\prime \prime}} \mathcal{D} \xi(t)\left(\xi^{2}+\eta^{2}\right) \int_{z\left(t^{\prime}\right)=z^{\prime}}^{z\left(t^{\prime \prime}\right)=z^{\prime \prime}} \mathcal{D} z(t) \\
& \quad \times \exp \left\{\frac { \mathrm { i } } { \hbar } \int _ { t ^ { \prime } } ^ { t ^ { \prime \prime } } \left[\frac{M}{2}\left(\left(\xi^{2}+\eta^{2}\right)\left(\dot{\xi}^{2}+\dot{\eta}^{2}\right)+\dot{z}^{2}\right)\right.\right.
\end{aligned}
$$




$$
\left.\left.-\frac{\hbar^{2}}{2 M}\left(\frac{k_{1}^{2}\left(\eta^{2}-\xi^{2}\right)}{\xi^{2} \eta^{2}\left(\xi^{2}+\eta^{2}\right)}+\frac{k_{2}^{2}-\frac{1}{4}}{\xi^{2} \eta^{2}}+\frac{k_{3}^{2}-\frac{1}{4}}{z^{2}}\right)\right] d t\right\} .
$$

We obtain for the path integral solutions $\left(\lambda_{ \pm}^{2}=k_{2}^{2} \pm k_{1}^{2}, \lambda_{1}=n+\left(\lambda_{+}+\lambda_{-}+1\right) / 2, \lambda_{2}=\right.$ $\left.2 m+\lambda_{1} \pm k_{3}+1\right)$ 
$K^{\left(V_{4}\right)}\left(\vec{x}^{\prime \prime}, \vec{x}^{\prime} ; T\right)$

Spherical Coordinates:

$$
\begin{aligned}
= & \frac{1}{2}\left(r^{\prime} r^{\prime \prime} \sin \theta^{\prime} \sin \theta^{\prime \prime}\right)^{-1 / 2} \sum_{n=0}^{\infty} \Psi_{n}^{\left(\lambda_{+}, \lambda_{-}\right)}\left(\frac{\phi^{\prime}}{2}\right) \Psi_{n}^{\left(\lambda_{+}, \lambda_{-}\right)}\left(\frac{\phi^{\prime \prime}}{2}\right) \\
& \times \frac{M}{\mathrm{i} \hbar T} \exp \left[\frac{\mathrm{i} M}{2 \hbar T}\left(r^{\prime 2}+r^{\prime \prime 2}\right)\right] \sum_{m=0}^{\infty} \Phi_{m}^{\left(\lambda_{1}, \pm k_{3}\right)}\left(\theta^{\prime}\right) \Phi_{m}^{\left(\lambda_{1}, \pm k_{3}\right)}\left(\theta^{\prime \prime}\right) I_{\lambda_{2}}\left(\frac{M r^{\prime} r^{\prime \prime}}{\mathrm{i} \hbar T}\right) \\
= & \sum_{n, m=0}^{\infty} \int_{0}^{\infty} d p \mathrm{e}^{-\mathrm{i} E_{p} T / \hbar} \Psi_{n, m, p}^{*}\left(\theta^{\prime}, \phi^{\prime}, r^{\prime}\right) \Psi_{n, m, p}\left(\theta^{\prime \prime}, \phi^{\prime \prime}, r^{\prime \prime}\right)
\end{aligned}
$$

Circular Polar Coordinates:

$$
\begin{aligned}
= & \left(\frac{M}{\mathrm{i} \hbar T}\right)^{2} \sqrt{z^{\prime} z^{\prime \prime}} \exp \left[\frac{\mathrm{i} M}{2 \hbar}\left(z^{\prime 2}+z^{\prime \prime 2}+\rho^{\prime 2}+\rho^{\prime \prime 2}\right)\right] I_{ \pm k_{3}}\left(\frac{M z^{\prime} z^{\prime \prime}}{\mathrm{i} \hbar T}\right) \\
& \times \frac{1}{2} \sum_{n=0}^{\infty} \Phi_{n}^{\left(\lambda_{+}, \lambda_{-}\right)}\left(\frac{\phi^{\prime}}{2}\right) \Phi_{n}^{\left(\lambda_{+}, \lambda_{-}\right)}\left(\frac{\phi^{\prime \prime}}{2}\right) I_{\lambda_{1}}\left(\frac{M \rho^{\prime} \rho^{\prime \prime}}{\mathrm{i} \hbar T}\right) \\
= & \sum_{n=0}^{\infty} \int_{0}^{\infty} d p_{z} \int_{0}^{\infty} d p \mathrm{e}^{-\mathrm{i} E_{p_{z}, p} T / \hbar} \Psi_{p_{z}, n, p}^{*}\left(\phi^{\prime}, \rho^{\prime}, z^{\prime}\right) \Psi_{p_{z}, n, p}\left(\phi^{\prime \prime}, \rho^{\prime \prime}, z^{\prime \prime}\right)
\end{aligned}
$$

Circular Parabolic Coordinates:

$$
\begin{aligned}
& =\frac{M}{\mathrm{i} \hbar T} \sqrt{z^{\prime} z^{\prime \prime}} \exp \left[\frac{\mathrm{i} M}{2 \hbar T}\left(z^{\prime 2}+z^{\prime \prime 2}\right)\right] I_{ \pm k_{3}}\left(\frac{M z^{\prime} z^{\prime \prime}}{\mathrm{i} \hbar T}\right) \\
& \quad \times \int_{\mathbb{R}} d \zeta \int_{0}^{\infty} \frac{d p}{p} \frac{\left|\Gamma\left(\frac{1+\lambda_{+}}{2}+\frac{\mathrm{i} \zeta}{2 p}\right)\right|^{2}\left|\Gamma\left(\frac{1+\lambda_{-}}{2}-\frac{\mathrm{i} \zeta}{2 p}\right)\right|^{2} \mathrm{e}^{\pi / a p}}{4 \pi^{2} \sqrt{\xi^{\prime} \xi^{\prime \prime} \eta^{\prime} \eta^{\prime \prime}} \Gamma^{2}\left(1+\lambda_{-}\right) \Gamma^{2}\left(1+\lambda_{+}\right)} \mathrm{e}^{-\mathrm{i} \hbar p^{2} T / 2 M} \\
& \quad \times M_{-\mathrm{i} \zeta / 2 p, \lambda_{+} / 2}\left(-\mathrm{i} p \xi^{\prime \prime 2}\right) M_{\mathrm{i} \zeta / 2 p, \lambda_{+} / 2}\left(\mathrm{i} p \xi^{2}\right) M_{\mathrm{i} \zeta / 2 p, \lambda_{-} / 2}\left(-\mathrm{i} p \eta^{\prime \prime 2}\right) M_{-\mathrm{i} \zeta / 2 p, \lambda_{-} / 2}\left(\mathrm{i} p \eta^{\prime 2}\right) \\
& =\int_{0}^{\infty} d p_{z} \int_{\mathbb{R}} d \zeta \int_{0}^{\infty} d p \mathrm{e}^{-\mathrm{i} E_{p_{z}, p} T / \hbar} \Psi_{p_{z}, p, \zeta}^{*}\left(z^{\prime}, \xi^{\prime}, \eta^{\prime}\right) \Psi_{p_{z}, p, \zeta}\left(z^{\prime \prime}, \xi^{\prime \prime}, \eta^{\prime \prime}\right),
\end{aligned}
$$

( $\zeta$ is the parabolic separation constant) where the wave-functions and the energy-spectra are given by

Spherical Coordinates:

$$
\begin{aligned}
& \Psi_{n, m, p}(\theta, \phi, r)=(2 r \sin \theta)^{-1 / 2} \Phi_{n}^{\left(\lambda_{+}, \lambda_{-}\right)}\left(\frac{\phi}{2}\right) \Phi_{m}^{\left(\lambda_{1}, \pm k_{3}\right)}(\theta) \sqrt{p} J_{\lambda_{2}}(p r), \\
& E_{p}=\frac{\hbar^{2}}{2 M} p^{2}
\end{aligned}
$$

Circular Polar Coordinates:

$$
\begin{aligned}
& \Psi_{n, p, p_{z}}(\phi, \rho, z)=\sqrt{p_{z} z p} J_{ \pm k_{3}}\left(p_{z} z\right) J_{\lambda_{1}}(p \rho) 2^{-1 / 2} \Phi_{n}^{\left(\lambda_{+}, \lambda_{-}\right)}\left(\frac{\phi}{2}\right), \\
& E_{p_{z}, p}=\frac{\hbar^{2}}{2 M}\left(p_{z}^{2}+p^{2}\right),
\end{aligned}
$$

Circular Parabolic Coordinates:

$$
\begin{gathered}
\Psi_{p_{z}, p, \zeta}(z, \xi, \eta)=\sqrt{p_{z} z} J_{ \pm k_{3}}\left(p_{z} z\right) \frac{\Gamma\left(\frac{1+\lambda_{+}}{2}+\frac{\mathrm{i} \zeta}{2 p}\right) \Gamma\left(\frac{1+\lambda_{-}}{2}-\frac{\mathrm{i} \zeta}{2 p}\right) \mathrm{e}^{\pi / 2 a p}}{2 \pi \sqrt{p \xi \eta} \Gamma\left(1+\lambda_{-}\right) \Gamma\left(1+\lambda_{+}\right)} \\
\times M_{-\mathrm{i} \zeta / 2 p, \lambda_{+} / 2}\left(-\mathrm{i} p \xi^{2}\right) M_{-\mathrm{i} \zeta / 2 p, \lambda_{-} / 2}\left(-\mathrm{i} p \eta^{2}\right)
\end{gathered}
$$

In the path integral evaluation subsequently [55] the path integral solution for the Pöschl-Teller potential and the radial harmonic oscillator have been used. The spectral expansion in the 
$z$ - and $\rho$-dependent kernels is done by means of (analytical continuation [113] of) the Weber formula

$$
\int_{0}^{\infty} r \mathrm{e}^{\mathrm{i} \alpha r^{2}} I_{\nu}(-\mathrm{i} a r) I_{\nu}(-\mathrm{i} b r) d r=\frac{\mathrm{i}}{2 \alpha} \mathrm{e}^{\left(a^{2}+b^{2}\right) / 4 \alpha i} I_{\nu}\left(\frac{a b}{2 \alpha \mathrm{i}}\right)
$$

and we obtain the path integral identity (we use the functional weight formulation)

$$
\begin{aligned}
\int_{r\left(t^{\prime}\right)=r^{\prime}}^{r\left(t^{\prime \prime}\right)=r^{\prime \prime}} \mu_{\lambda}\left[r^{2}\right] \mathcal{D} r(t) \exp \left(\frac{\mathrm{i} M}{2 \hbar} \int_{t^{\prime}}^{t^{\prime \prime}} \dot{r}^{2} d t\right) & =\sqrt{r^{\prime} r^{\prime \prime}} \frac{M}{\mathrm{i} \hbar T} \exp \left[\frac{\mathrm{i} M}{2 \hbar T}\left(r^{\prime 2}+r^{\prime \prime 2}\right)\right] I_{\lambda}\left(\frac{M r^{\prime} r^{\prime \prime}}{\mathrm{i} \hbar T}\right) \\
& =\sqrt{r^{\prime} r^{\prime \prime}} \int_{0}^{\infty} p d p \mathrm{e}^{-\mathrm{i} \hbar p^{2} T / 2 M} J_{\lambda}\left(p r^{\prime}\right) J_{\lambda}\left(p r^{\prime \prime}\right)
\end{aligned}
$$

Applying (234) gives in an obvious way the spectral expansion of the $z$ - and $\rho$-dependent kernel. For the extraction of the continuous states in parabolic one applies (135) by introducing a Coulomb coupling $\alpha$ and sets $a=\infty$, i.e. infinite Bohr radius, in the in the final formulæ.

In circular elliptic II coordinates the $z$-path integration is separated in the same way as for the circular parabolic case, and for the remaining path integrations we obtain the identity

$$
\begin{aligned}
& K^{\left(V_{4}\right)}\left(\vec{x}^{\prime \prime}, \vec{x}^{\prime} ; T\right) \\
& =\frac{M}{\mathrm{i} \hbar T} \sqrt{z^{\prime} z^{\prime \prime}} \exp \left[\frac{\mathrm{i} M}{2 \hbar T}\left(z^{\prime 2}+z^{\prime \prime 2}\right)\right] I_{ \pm k_{3}}\left(\frac{M z^{\prime} z^{\prime \prime}}{\mathrm{i} \hbar T}\right) \\
& \times \int_{\mathbb{R}} \frac{d E}{2 \pi \hbar} \mathrm{e}^{-\mathrm{i} E T / \hbar} \int_{0}^{\infty} d s^{\prime \prime} \int_{\xi\left(s^{\prime \prime}\right)=\xi^{\prime \prime}} \mathcal{D} \xi(s) \int_{\eta(0)=\eta^{\prime}}^{\prime \prime} \mathcal{D} \eta(s) \\
& \quad \times \exp \left\{\frac { \mathrm { i } } { \hbar } \int _ { 0 } ^ { s ^ { \prime \prime } } \left[\frac{M}{2}\left(\dot{\xi}^{2}+\dot{\eta}^{2}\right)+E d^{2}\left(\cosh ^{2} \xi-\cos ^{2} \eta\right)\right.\right. \\
& \left.\left.\quad-\frac{\hbar^{2}}{2 M}\left(\frac{\left(k_{2}^{2}-\frac{1}{2}\right)+k_{1}^{2} \cos \eta}{\sin ^{2} \eta}+\frac{\left(k_{2}^{2}-\frac{1}{2}\right)+k_{1}^{2} \cosh \xi}{\sinh ^{2} \xi}\right)\right] d s\right\} .
\end{aligned}
$$

For the spherical coordinates the expansion into radial wave-functions, and for the circular parabolic coordinates the expansion of the $z$-dependent kernel are performed by means of the Hille-Hardy formula (93).

3.2.5. We consider the potential $\left(k_{1,2}>0\right)$

$$
V_{5}(\vec{x})=\frac{\hbar^{2}}{2 M}\left(\frac{k_{1}^{2} x}{y^{2} \sqrt{x^{2}+y^{2}}}+\frac{k_{2}^{2}-\frac{1}{4}}{y^{2}}\right)-k_{3} z .
$$

Note that for $k_{3}=0$ we obtain a trivial extension of the $\alpha=0$ two-dimensional maximally super-integrable potential $V_{3}(\vec{x})$. We obtain the path integral formulations

$$
K^{\left(V_{5}\right)}\left(\vec{x}^{\prime \prime}, \vec{x}^{\prime} ; T\right)
$$

\section{Circular Polar Coordinates:}

$$
\begin{aligned}
& =\int_{\rho\left(t^{\prime}\right)=\rho^{\prime}}^{\rho\left(t^{\prime \prime}\right)=\rho^{\prime \prime}} \rho \mathcal{D} \rho(t) \int_{\phi\left(t^{\prime}\right)=\phi^{\prime}}^{\phi\left(t^{\prime \prime}\right)=\phi^{\prime \prime}} \mathcal{D} \phi(t) \int_{z\left(t^{\prime}\right)=z^{\prime}}^{z\left(t^{\prime \prime}\right)=z^{\prime \prime}} \mathcal{D} z(t) \\
& \quad \times \exp \left\{\frac{\mathrm{i}}{\hbar} \int_{t^{\prime}}^{t^{\prime \prime}}\left[\frac{M}{2}\left(\dot{\rho}^{2}+\rho^{2} \dot{\phi}^{2}+\dot{z}^{2}\right)-k_{3} z-\frac{\hbar^{2}}{2 M \rho^{2}}\left(\frac{k_{2}^{2}-k_{1}^{2}-\frac{1}{4}}{4 \cos ^{2} \frac{\phi}{2}}+\frac{k_{2}^{2}+k_{1}^{2}-\frac{1}{4}}{4 \sin ^{2} \frac{\phi}{2}}-\frac{1}{4}\right)\right] d t\right\}
\end{aligned}
$$


Circular Elliptic II Coordinates:

$$
\begin{aligned}
& =\int_{\xi\left(t^{\prime}\right)=\xi^{\prime}}^{\xi\left(t^{\prime \prime}\right)=\xi^{\prime \prime}} \mathcal{D} \xi(t) \int_{\eta\left(t^{\prime}\right)=\eta^{\prime}}^{\eta\left(t^{\prime \prime}\right)=\eta^{\prime \prime}} \mathcal{D} \eta(t) d^{2}\left(\sinh ^{2} \xi+\sin ^{2} \eta\right) \int_{z\left(t^{\prime}\right)=z^{\prime}}^{z\left(t^{\prime \prime}\right)=z^{\prime \prime}} \mathcal{D} z(t) \\
& \times \exp \left\{\frac { \mathrm { i } } { \hbar } \int _ { t ^ { \prime } } ^ { t ^ { \prime \prime } } \left[\frac{M}{2}\left(\left(\sinh ^{2} \xi^{2}+\sin ^{2} \eta\right)\left(\dot{\xi}^{2}+\dot{\eta}^{2}\right)+\dot{z}^{2}\right)-k_{3} z\right.\right. \\
& \left.\left.-\frac{\hbar^{2}}{2 M} \frac{1}{d^{2} \sinh ^{2} \xi \sin ^{2} \eta}\left(k_{1}^{2} \frac{\cosh \xi \cos \eta+1}{\cosh \xi+\cos \eta}+k_{2}^{2}-\frac{1}{2}\right)\right] d t\right\}
\end{aligned}
$$

Circular Parabolic Coordinates:

$$
\begin{aligned}
& =\int_{\eta\left(t^{\prime}\right)=\eta^{\prime}}^{\eta\left(t^{\prime \prime}\right)=\eta^{\prime \prime}} \mathcal{D} \eta(t) \int_{\xi\left(t^{\prime}\right)=\xi^{\prime}}^{\xi\left(t^{\prime \prime}\right)=\xi^{\prime \prime}} \mathcal{D} \xi(t)\left(\xi^{2}+\eta^{2}\right) \int_{z\left(t^{\prime}\right)=z^{\prime}}^{z\left(t^{\prime \prime}\right)=z^{\prime \prime}} \mathcal{D} z(t) \\
& \quad \times \exp \left\{\frac{\mathrm{i}}{\hbar} \int_{t^{\prime}}^{t^{\prime \prime}}\left[\frac{M}{2}\left(\left(\xi^{2}+\eta^{2}\right)\left(\dot{\xi}^{2}+\dot{\eta}^{2}\right)+\dot{z}^{2}\right)-\frac{\hbar^{2}}{2 M}\left(k_{1}^{2} \frac{\eta^{2}-\xi^{2}}{\xi^{2} \eta^{2}\left(\xi^{2}+\eta^{2}\right)}+\frac{k_{2}^{2}-\frac{1}{4}}{\xi^{2} \eta^{2}}\right)-k_{3} z\right] d t\right\}
\end{aligned}
$$

$\underline{\text { Parabolic Coordinates }}\left(\lambda^{2}=k_{1}^{2}+k_{2}^{2}\right)$ :

$$
\begin{aligned}
& =\int_{\eta\left(t^{\prime}\right)=\eta^{\prime}}^{\eta\left(t^{\prime \prime}\right)=\eta^{\prime \prime}} \mathcal{D} \eta(t) \int_{\xi\left(t^{\prime}\right)=\xi^{\prime}}^{\xi\left(t^{\prime \prime}\right)=\xi^{\prime \prime}} \mathcal{D} \xi(t)\left(\xi^{2}+\eta^{2}\right) \xi \eta \int_{\phi\left(t^{\prime}\right)=\phi^{\prime}}^{\phi\left(t^{\prime \prime}\right)=\phi^{\prime \prime}} \mathcal{D} \phi(t) \\
& \times \exp \left\{\frac { \mathrm { i } } { \hbar } \int _ { t ^ { \prime } } ^ { t ^ { \prime \prime } } \left[\frac{M}{2}\left(\left(\xi^{2}+\eta^{2}\right)\left(\dot{\xi}^{2}+\dot{\eta}^{2}\right)+\xi^{2} \eta^{2} \dot{\phi}^{2}\right)\right.\right. \\
& \left.\left.-\frac{\hbar^{2}}{2 M \xi^{2} \eta^{2}}\left(\frac{k_{1}^{2}+k_{2}^{2}-\frac{1}{4}}{\sin ^{2} \phi}-k_{1}^{2}-\frac{1}{4}\right)-\frac{k_{3}}{2}\left(\xi^{2}-\eta^{2}\right)\right] d t\right\} \\
& =\left(\xi^{\prime} \xi^{\prime \prime} \eta^{\prime} \eta^{\prime \prime}\right)^{-1 / 2} \sum_{n=0}^{\infty}\left(n+\lambda+\frac{1}{2}\right) \frac{\Gamma(n+2 \lambda+1)}{n !} P_{\lambda+n}^{-\lambda}\left(\cos \phi^{\prime}\right) P_{\lambda+n}^{-\lambda}\left(\cos \phi^{\prime \prime}\right) \\
& \times \int_{\mathbb{R}} \frac{d E}{2 \pi \hbar} \mathrm{e}^{-\mathrm{i} E T / \hbar} \int_{0}^{\infty} d s^{\prime \prime} \int_{\eta(0)=\eta^{\prime}}^{\eta\left(s^{\prime \prime}\right)=\eta^{\prime \prime}} \mathcal{D} \eta(s) \int_{\xi(0)=\xi^{\prime}}^{\xi\left(s^{\prime \prime}\right)=\xi^{\prime \prime}} \mathcal{D} \xi(s) \\
& \times \exp \left\{\frac { \mathrm { i } } { \hbar } \int _ { 0 } ^ { s ^ { \prime \prime } } \left[\frac{M}{2}\left(\dot{\xi}^{2}+\dot{\eta}^{2}\right)-\frac{k_{3}}{2}\left(\xi^{4}-\eta^{4}\right)+E\left(\xi^{2}+\eta^{2}\right)\right.\right. \\
& \left.\left.-\frac{\hbar^{2}}{2 M}\left(\frac{\lambda^{2}-k_{1}^{2}-\frac{1}{4}}{\xi^{2}}+\frac{\lambda^{2}-k_{1}^{2}-\frac{1}{4}}{\eta^{2}}\right)\right] d s\right\} .
\end{aligned}
$$

The path integral in parabolic coordinates cannot be done due to the $\xi^{4}, \eta^{4}$ dependence. Actually $V_{5}$ represents a Stark effect. In the cases of the circular polar and circular parabolic coordinates the corresponding Green function can be evaluated by means of the path integral solution for the linear potential [38] for the $z$-path integration, and the radial harmonic oscillator in the $(r, \phi)$, and in the $\xi$ - and $\eta$-path integration $\left[\lambda_{ \pm}^{2}=k_{2}^{2} \pm k_{1}^{2}, \lambda_{1}=n+\left(\lambda_{+}+\lambda_{-}+1\right) / 2\right]$

$$
\begin{aligned}
& K^{\left(V_{5}\right)}\left(\vec{x}^{\prime \prime}, \vec{x}^{\prime} ; T\right) \\
& \text { Circular Polar Coordinates: } \\
& =\left(\frac{M}{2 \pi \mathrm{i} \hbar T}\right)^{1 / 2} \exp \left[\frac{\mathrm{i}}{\hbar}\left(\frac{M}{2 T}\left(z^{\prime \prime}-z^{\prime}\right)^{2}-\frac{k_{3} T}{2}\left(z^{\prime}+z^{\prime \prime}\right)-\frac{k_{3}^{2} T^{3}}{24 M}\right)\right]
\end{aligned}
$$




$$
\begin{aligned}
& \quad \times \frac{M}{2 \mathrm{i} \hbar T} \sum_{n=0}^{\infty} \Phi_{n}^{\left(\lambda_{+}, \lambda_{-}\right)}\left(\frac{\phi^{\prime}}{2}\right) \Phi_{n}^{\left(\lambda_{+}, \lambda_{-}\right)}\left(\frac{\phi^{\prime \prime}}{2}\right) \exp \left[-\frac{M}{2 \mathrm{i} \hbar T}\left(\rho^{\prime 2}+\rho^{\prime \prime 2}\right)\right] I_{\lambda_{1}}\left(\frac{M \rho^{\prime} \rho^{\prime \prime}}{\mathrm{i} \hbar T}\right) \\
& =\sum_{n=0}^{\infty} \int_{0}^{\infty} d p \int_{\mathbb{R}} d E_{z} \mathrm{e}^{-\mathrm{i} E_{E_{z}, n, p} T / \hbar} \Psi_{E_{z}, n, p}^{*}\left(z^{\prime}, \phi^{\prime}, \rho^{\prime}\right) \Psi_{E_{z}, n, p}\left(z^{\prime \prime}, \phi^{\prime \prime}, \rho^{\prime \prime}\right) \\
& \text { Circular Parabolic Coordinates: } \\
& =\left(\frac{M}{2 \pi \mathrm{i} \hbar T}\right)^{1 / 2} \exp \left[\frac{\mathrm{i}}{\hbar}\left(\frac{M}{2 T}\left(z^{\prime \prime}-z^{\prime}\right)^{2}-\frac{k_{3} T}{2}\left(z^{\prime}+z^{\prime \prime}\right)-\frac{k_{3}^{2} T^{3}}{24 M}\right)\right] \\
& \quad \times \int_{\mathbb{R}} d \zeta \int_{0}^{\infty} \frac{d p}{p} \frac{\left|\Gamma\left(\frac{1+\lambda_{+}}{2}+\frac{\mathrm{i} \zeta}{2 p}\right)\right|^{2}\left|\Gamma\left(\frac{1+\lambda_{-}}{2}-\frac{\mathrm{i} \zeta}{2 p}\right)\right|^{2} \mathrm{e}^{\pi / a p} \mathrm{r}_{\xi^{\prime} \xi^{\prime \prime} \eta^{\prime} \eta^{\prime \prime}} \Gamma^{2}\left(1+\lambda_{-}\right) \Gamma^{2}\left(1+\lambda_{+}\right)}{\mathrm{e}^{2} \hbar p^{2} T / 2 M} \\
& \quad \times M_{-\mathrm{i} \zeta / 2 p, \lambda_{+} / 2}\left(-\mathrm{i} p \xi^{\prime \prime 2}\right) M_{\mathrm{i} \zeta / 2 p, \lambda_{+} / 2}\left(\mathrm{i} p \xi^{\prime \prime 2}\right) M_{\mathrm{i} \zeta / 2 p, \lambda_{-} / 2}\left(-\mathrm{i} p \eta^{\prime \prime 2}\right) M_{-\mathrm{i} \zeta / 2 p, \lambda_{-} / 2}\left(\mathrm{i} p \eta^{\prime \prime 2}\right) \\
& =\int_{\mathbb{R}} d \zeta \int_{0}^{\infty} d p \int_{\mathbb{R}} d E_{z} \mathrm{e}^{-\mathrm{i} E_{E_{z}, p} T / \hbar} \Psi_{E_{z}, p, \zeta}^{*}\left(z^{\prime}, \xi^{\prime}, \eta^{\prime}\right) \Psi_{E_{z}, p, \zeta}\left(z^{\prime \prime}, \xi^{\prime \prime}, \eta^{\prime \prime}\right),
\end{aligned}
$$

( $\zeta$ is the parabolic separation constant) where the wave-functions and the energy-spectra are given by

Circular Polar Coordinates:

$$
\begin{aligned}
& \Psi_{E_{z}, n, p}(z, \phi, \rho)=\left(\frac{2 M}{\hbar^{2} \sqrt{k}}\right)^{1 / 3} \mathrm{Ai}\left[\left(z-\frac{E_{z}}{k}\right)\left(\frac{2 M k}{\hbar^{2}}\right)^{1 / 3}\right] 2^{-1 / 2} \Phi_{n}^{\left(\lambda_{+}, \lambda_{-}\right)}\left(\frac{\phi}{2}\right) \sqrt{p \rho} J_{\lambda_{1}}(p \rho), \\
& E_{E_{z}, p}=E_{z}+\frac{\hbar^{2}}{2 M} p^{2}
\end{aligned}
$$

Circular Parabolic Coordinates:

$$
\begin{aligned}
& \Psi_{E_{z}, p, \zeta}(z, \xi, \eta)=\left(\frac{2 M}{\hbar^{2} \sqrt{k}}\right)^{1 / 3} \mathrm{Ai}\left[\left(z-\frac{E_{z}}{k}\right)\left(\frac{2 M k}{\hbar^{2}}\right)^{1 / 3}\right] \\
& \times \frac{\Gamma\left(\frac{1+\lambda_{+}}{2}+\frac{\mathrm{i} \zeta}{2 p}\right) \Gamma\left(\frac{1+\lambda_{-}}{2}-\frac{\mathrm{i} \zeta}{2 p}\right) \mathrm{e}^{\pi / 2 a p}}{2 \pi \sqrt{p \xi \eta} \Gamma\left(1+\lambda_{-}\right) \Gamma\left(1+\lambda_{+}\right)} M_{-\mathrm{i} \zeta / 2 p, \lambda_{+} / 2}\left(-\mathrm{i} p \xi^{2}\right) M_{\mathrm{i} \zeta / 2 p, \lambda_{-} / 2}\left(-\mathrm{i} p \eta^{2}\right) \\
& E_{E_{z}, p}=E_{z}+\frac{\hbar^{2}}{2 M} p^{2} .
\end{aligned}
$$

$\zeta$ is the parabolic separation constant.

The expansion into the wave-function in the $z$ coordinates is done according to $([38], \operatorname{Ai}(z)$ are Airy functions)

$$
\begin{aligned}
& \int_{z\left(t^{\prime}\right)=z^{\prime}}^{z\left(t^{\prime \prime}\right)=z^{\prime \prime}} \mathcal{D} z(t) \exp \left[\frac{\mathrm{i}}{\hbar} \int_{t^{\prime}}^{t^{\prime \prime}}\left(\frac{m}{2} \dot{z}^{2}-k z\right) d t\right] \\
& =\left(\frac{M}{2 \pi \mathrm{i} \hbar T}\right)^{1 / 2} \exp \left[\frac{\mathrm{i}}{\hbar}\left(\frac{M}{2 T}\left(z^{\prime \prime}-z^{\prime}\right)^{2}-\frac{k T}{2}\left(z^{\prime}+z^{\prime \prime}\right)-\frac{k^{2} T^{3}}{24 M}\right)\right] \\
& =\int_{\mathbb{R}} d E \mathrm{e}^{-\mathrm{i} E T / \hbar}\left(\frac{2 M}{\hbar^{2} \sqrt{k}}\right)^{2 / 3} \mathrm{Ai}\left[\left(z^{\prime}-\frac{E}{k}\right)\left(\frac{2 M k}{\hbar^{2}}\right)^{1 / 3}\right] \mathrm{Ai}\left[\left(z^{\prime \prime}-\frac{E}{k}\right)\left(\frac{2 M k}{\hbar^{2}}\right)^{1 / 3}\right] .
\end{aligned}
$$

The expansion into the radial wave-functions for the circular polar coordinates is performed by the Weber formula (234).

\subsection{Three-Dimensional Minimally Super-Integrable Smorodinsky-Winternitz Po- tentials.}

We discuss in this subsection the eight three-dimensional minimally super-integrable potentials. 
The are characterized by having four functionally independent integrals of motion. In the sequel $\vec{x}$ denotes a three-dimensional coordinate: $\vec{x}=(x, y, z) \equiv\left(x_{1}, x_{2}, x_{3}\right)$.

In table 4 we list the three-dimensional minimally super-integrable Smorodinsky-Winternitz potentials together with the separating coordinate systems (where the italized coordinates systems were not mentioned in [35]. The cases where an explicit path integration is possible are underlined.

Table 4: The three-dimensional minimally super-integrable potentials

\begin{tabular}{|c|c|}
\hline Potential $V(x, y, z)$ & Coordinate System \\
\hline$V_{1}=F(r)+\frac{\hbar^{2}}{2 M}\left(\frac{k_{1}^{2}-\frac{1}{4}}{x^{2}}+\frac{k_{2}^{2}-\frac{1}{4}}{y^{2}}+\frac{k_{3}^{2}-\frac{1}{4}}{z^{2}}\right)$ & $\frac{\text { Spherical }}{\text { Conical }}$ \\
\hline$V_{2}=\frac{M}{2} \omega^{2}\left(x^{2}+y^{2}\right)+\frac{\hbar^{2}}{2 M}\left(\frac{k_{1}^{2}-\frac{1}{4}}{x^{2}}+\frac{k_{2}^{2}-\frac{1}{4}}{y^{2}}\right)+F(z)$ & $\begin{array}{l}\frac{\text { Cartesian }}{\text { Circular Polar }} \\
\text { Circular Elliptic }\end{array}$ \\
\hline$V_{3}=\frac{M}{2} \omega^{2}\left(4 x^{2}+y^{2}\right)+\frac{\hbar^{2}}{2 M} \frac{k_{2}^{2}-\frac{1}{4}}{y^{2}}+F(z)$ & $\frac{\text { Cartesian }}{\text { Circular Parabolic }}$ \\
\hline$V_{4}=-\frac{\alpha}{\sqrt{x^{2}+y^{2}}}+\frac{\hbar^{2}}{2 M}\left(\frac{k_{1}^{2} x}{y^{2} \sqrt{x^{2}+y^{2}}}+\frac{k_{2}^{2}-\frac{1}{4}}{y^{2}}\right)+F(z)$ & $\begin{array}{l}\text { Circular Polar } \\
\text { Circular Elliptic II } \\
\text { Circular Parabolic } \\
\end{array}$ \\
\hline$V_{5}=\frac{M}{2} \omega^{2}\left(x^{2}+y^{2}+z^{2}\right)+\frac{\hbar^{2}}{2 M}\left(\frac{k_{3}^{2}-\frac{1}{4}}{z^{2}}+\frac{F(y / x)}{x^{2}+y^{2}}\right)$ & $\begin{array}{l}\frac{\text { Spherical }}{\text { Circular Polar }} \\
\text { Oblate Spheroidal } \\
\text { Prolate Spheroidal }\end{array}$ \\
\hline$V_{6}=\frac{M}{2} \omega^{2}\left(x^{2}+y^{2}+4 z^{2}\right)+\frac{\hbar^{2}}{2 M} \frac{F(y / x)}{x^{2}+y^{2}}$ & $\frac{\text { Circular Polar }}{\text { Parabolic }}$ \\
\hline$V_{7}=-\frac{\alpha}{r}+\frac{\hbar^{2}}{2 M\left(x^{2}+y^{2}\right)}\left(\frac{k_{1}^{2} z}{r}+F\left(\frac{y}{x}\right)\right)$ & $\begin{array}{l}\frac{\text { Spherical }}{\text { Parabolic }} \\
\text { Prolate Spheroidal II }\end{array}$ \\
\hline$V_{8}=-\frac{\alpha}{\rho}+\sqrt{\frac{2}{\rho}}\left(\beta_{1} \cos \frac{\phi}{2}+\beta_{2} \sin \frac{\phi}{2}\right)+F(z)$ & $\begin{array}{l}\text { Mutually Circular } \\
\text { Parabolic }\end{array}$ \\
\hline
\end{tabular}

3.3.1. We consider the potential $\left(r=|\vec{x}|, k_{1,2,3}>0\right)$

$$
V_{1}(\vec{x})=F(r)+\frac{\hbar^{2}}{2 M}\left(\frac{k_{1}^{2}-\frac{1}{4}}{x^{2}}+\frac{k_{2}^{2}-\frac{1}{4}}{y^{2}}+\frac{k_{3}^{2}-\frac{1}{4}}{z^{2}}\right) \text {. }
$$


We formulate the path integral in the separating coordinate systems and obtain

$$
\begin{aligned}
& K^{\left(V_{1}\right)}\left(\vec{x}^{\prime \prime}, \vec{x}^{\prime} ; T\right) \\
& =\int_{\vec{x}\left(t^{\prime}\right)=\vec{x}^{\prime}}^{\vec{x}\left(t^{\prime \prime}\right)=\vec{x}^{\prime \prime}} \mathcal{D} \vec{x}(t) \exp \left\{\frac{\mathrm{i}}{\hbar} \int_{t^{\prime}}^{t^{\prime \prime}}\left[\frac{M}{2} \dot{\vec{x}}^{2}-F(r)-\frac{\hbar^{2}}{2 M}\left(\frac{k_{1}^{2}-\frac{1}{4}}{x^{2}}+\frac{k_{2}^{2}-\frac{1}{4}}{y^{2}}+\frac{k_{3}^{2}-\frac{1}{4}}{z^{2}}\right)\right] d t\right\}
\end{aligned}
$$

$\underline{\text { Spherical Coordinates: }}$

$$
\begin{aligned}
& =\int_{r\left(t^{\prime}\right)=r^{\prime}}^{r\left(t^{\prime \prime}\right)=r^{\prime \prime}} r^{2} \mathcal{D} r(t) \int_{\theta\left(t^{\prime}\right)=\theta^{\prime}}^{\theta\left(t^{\prime \prime}\right)=\theta^{\prime \prime}} \sin \theta \mathcal{D} \theta(t) \int_{\phi\left(t^{\prime}\right)=\phi^{\prime}}^{\phi\left(t^{\prime \prime}\right)=\phi^{\prime \prime}} \mathcal{D} \phi(t) \\
& \quad \times \exp \left\{\frac { \mathrm { i } } { \hbar } \int _ { t ^ { \prime } } ^ { t ^ { \prime \prime } } \left[\frac{M}{2}\left(\dot{r}^{2}+r^{2} \dot{\theta}^{2}+r^{2} \sin ^{2} \theta \dot{\phi}^{2}\right)-F(r)\right.\right. \\
& \left.\left.\quad-\frac{\hbar^{2}}{2 M r^{2}}\left(\frac{1}{\sin ^{2} \theta}\left(\frac{k_{1}^{2}-\frac{1}{4}}{\cos ^{2} \phi}+\frac{k_{2}^{2}-\frac{1}{4}}{\sin ^{2} \phi}-\frac{1}{4}\right)+\frac{k_{3}^{2}-\frac{1}{4}}{\cos ^{2} \theta}-\frac{1}{4}\right)\right] d t\right\}
\end{aligned}
$$

Conical Coordinates:

$$
\begin{aligned}
& =\int_{r\left(t^{\prime}\right)=r^{\prime}}^{r\left(t^{\prime \prime}\right)=r^{\prime \prime}} r^{2} \mathcal{D} r(t) \int_{\alpha\left(t^{\prime}\right)=\alpha^{\prime}}^{\alpha\left(t^{\prime \prime}\right)=\alpha^{\prime \prime}} \mathcal{D} \alpha(t) \int_{\beta\left(t^{\prime}\right)=\beta^{\prime}}^{\beta\left(t^{\prime \prime}\right)=\beta^{\prime \prime}} \mathcal{D} \beta(t)\left(k^{2} \operatorname{cn}^{2} \alpha+k^{\prime 2} \mathrm{cn}^{2} \beta\right) \\
& \quad \times \exp \left\{\frac { \mathrm { i } } { \hbar } \int _ { t ^ { \prime } } ^ { t ^ { \prime \prime } } \left[\frac{M}{2}\left(\dot{r}^{2}+r^{2}\left(k^{2} \mathrm{cn}^{2} \alpha+k^{\prime 2} \mathrm{cn}^{2} \beta\right)\left(\dot{\alpha}^{2}+\dot{\beta}^{2}\right)\right)-F(r)\right.\right. \\
& \left.\left.\quad-\frac{\hbar^{2}}{2 M r^{2}}\left(\frac{k_{1}^{2}-\frac{1}{4}}{\operatorname{sn}^{2} \alpha \operatorname{dn}^{2} \beta}+\frac{k_{2}^{2}-\frac{1}{4}}{\operatorname{cn}^{2} \alpha \mathrm{cn}^{2} \beta}+\frac{k_{3}^{2}-\frac{1}{4}}{\mathrm{dn}^{2} \alpha \operatorname{sn}^{2} \beta}\right)\right] d t\right\} .
\end{aligned}
$$

For this potential the angular path integrations can be easily done and one is left with a radial path integral [10], i.e. $\left(\lambda_{1}=2 n \pm k_{1} \pm k_{2}+1, \lambda_{2}=2 m+\lambda_{1} \pm k_{3}+1\right)$

$$
\begin{aligned}
& K^{\left(V_{1}\right)}\left(\vec{x}^{\prime \prime}, \vec{x}^{\prime} ; T\right) \\
& =\left(r^{\prime 2} r^{\prime \prime 2} \sin \theta^{\prime} \sin \theta^{\prime \prime}\right)^{-1 / 2} \sum_{n=0}^{\infty} \Phi_{n}^{\left( \pm k_{2}, \pm k_{1}\right)}\left(\phi^{\prime \prime}\right) \Phi_{n}^{\left( \pm k_{2}, \pm k_{1}\right)}\left(\phi^{\prime}\right) \sum_{m=0}^{\infty} \Phi_{m}^{\left(\lambda_{1}, \pm k_{3}\right)}\left(\theta^{\prime \prime}\right) \Phi_{m}^{\left(\lambda_{1}, \pm k_{3}\right)}\left(\theta^{\prime}\right) \\
& \quad \times \int_{r\left(t^{\prime}\right)=r^{\prime \prime}}^{r\left(t^{\prime \prime}\right)=r^{\prime \prime}} \mathcal{D} r(t) \exp \left[\frac{\mathrm{i}}{\hbar} \int_{t^{\prime}}^{t^{\prime \prime}}\left(\frac{M}{2} \dot{r}^{2}-F(r)-\frac{\hbar^{2}}{2 M} \frac{\lambda_{2}^{2}-\frac{1}{4}}{r^{2}}\right) d t\right] \\
& \equiv\left(r^{\prime 2} r^{\prime \prime 2} \sin \theta^{\prime} \sin \theta^{\prime \prime}\right)^{-1 / 2} \sum_{n=0}^{\infty} \Phi_{n}^{\left( \pm k_{2}, \pm k_{1}\right)}\left(\phi^{\prime \prime}\right) \Phi_{n}^{\left( \pm k_{2}, \pm k_{1}\right)}\left(\phi^{\prime}\right) \sum_{m=0}^{\infty} \Phi_{m}^{\left(\lambda_{1}, \pm k_{3}\right)}\left(\theta^{\prime \prime}\right) \Phi_{m}^{\left(\lambda_{1}, \pm k_{3}\right)}\left(\theta^{\prime}\right) \\
& \quad \times \int_{r\left(t^{\prime \prime}\right)=r^{\prime \prime}} \mu_{\lambda_{2}}\left[r^{2}\right] \mathcal{D} r(t) \exp \left[\frac{\mathrm{i}}{\hbar} \int_{t^{\prime}}^{t^{\prime \prime}}\left(\frac{M}{2} \dot{r}^{2}-F(r)\right) d t\right] \\
& =\sum_{m, n=0}^{\infty} \int_{m} d E_{\lambda_{r}} \mathrm{e}^{-\mathrm{i} E_{\lambda_{r}} / \hbar} \Phi_{\lambda_{r}, n, m}^{*}\left(\theta^{\prime}, \phi^{\prime}, r^{\prime}\right) \Phi_{\lambda_{r}, n, m}\left(\theta^{\prime \prime}, \phi^{\prime \prime}, r^{\prime \prime}\right) .
\end{aligned}
$$

Here use has been made of the functional weight formulation in path integrals according to [59, 61, 119]. The wave-functions have the form

$$
\Phi_{\lambda_{r}, n, l,}(\theta, \phi, r)=\left(r^{2} \sin \theta\right)^{-1 / 2} \Phi_{n}^{\left( \pm k_{2}, \pm k_{1}\right)}(\phi) \Phi_{m}^{\left(\lambda_{1}, \pm k_{3}\right)}(\theta) \Phi_{\lambda_{r}}(r)
$$

where $\Phi_{\lambda_{r}}(r)$ are the radial wave-functions. Of special interest are the cases $F(r)=m \omega^{2} r^{2} / 2$ and $F(r)=-\alpha / r$, c.f. 3.2 .1 and 3.2 .3 , respectively. 
3.3.2. The next three potentials represent a simple $z$-dependence extension of the two-dimensional potentials $V_{1}-V_{3}$. We consider the potential $\left(k_{1,2}>0\right)$

$$
V_{2}(\vec{x})=\frac{M}{2} \omega^{2}\left(x^{2}+y^{2}\right)+\frac{\hbar^{2}}{2 M}\left(\frac{k_{1}^{2}-\frac{1}{4}}{x^{2}}+\frac{k_{2}^{2}-\frac{1}{4}}{y^{2}}\right)+F(z) .
$$

For the case of $F(z)=2 M \omega^{2} z^{2}$ there is an additional coordinate system, the parabolic coordinates, which separate $V_{2}$, c.f. the three-dimensional maximally super-integrable potential $V_{2}$. Here now we have

$$
K^{\left(V_{2}\right)}\left(\vec{x}^{\prime \prime}, \vec{x}^{\prime} ; T\right)
$$

\section{Cartesian Coordinates:}

$$
=\int_{\vec{x}\left(t^{\prime}\right)=\vec{x}^{\prime}}^{\vec{x}\left(t^{\prime \prime}\right)=\vec{x}^{\prime \prime}} \mathcal{D} \vec{x}(t) \exp \left\{\frac{\mathrm{i}}{\hbar} \int_{t^{\prime}}^{t^{\prime \prime}}\left[\frac{M}{2}\left(\dot{\vec{x}}^{2}-\omega^{2}\left(x^{2}+y^{2}\right)\right)-\frac{\hbar^{2}}{2 M} \sum_{i=1}^{2} \frac{k_{i}^{2}-\frac{1}{4}}{x_{i}^{2}}-F(z)\right] d t\right\}
$$

Circular Polar Coordinates:

$$
\begin{aligned}
& \int_{z\left(t^{\prime}\right)=z^{\prime}}^{z\left(t^{\prime \prime}\right)=z^{\prime \prime}} \mathcal{D} z(t) \int_{\rho\left(t^{\prime}\right)=\rho^{\prime}}^{\rho\left(t^{\prime \prime}\right)=\rho^{\prime \prime}} \rho \mathcal{D} \rho(t) \int_{\phi\left(t^{\prime}\right)=\phi^{\prime}}^{\phi\left(t^{\prime \prime}\right)=\phi^{\prime \prime}} \mathcal{D} \phi(t) \\
& \times \exp \left\{\frac{\mathrm{i}}{\hbar} \int_{t^{\prime}}^{t^{\prime \prime}}\left[\frac{M}{2}\left(\dot{\rho}^{2}+\rho^{2} \dot{\phi}^{2}-\omega^{2} \rho^{2}\right)-\frac{\hbar^{2}}{2 M \rho^{2}}\left(\frac{k_{1}^{2}-\frac{1}{4}}{\cos ^{2} \phi}+\frac{k_{2}^{2}-\frac{1}{4}}{\sin ^{2} \phi}-\frac{1}{4}\right)-F(z)\right] d t\right\}
\end{aligned}
$$

Circular Elliptic Coordinates:

$$
\begin{gathered}
=\int_{z\left(t^{\prime}\right)=z^{\prime}}^{z\left(t^{\prime \prime}\right)=z^{\prime \prime}} \mathcal{D} z(t) \int_{\xi\left(t^{\prime}\right)=\xi^{\prime}}^{\xi\left(t^{\prime \prime}\right)=\xi^{\prime \prime}} \mathcal{D} \xi(t) \int_{\eta\left(t^{\prime}\right)=\eta^{\prime}}^{\eta\left(t^{\prime \prime}\right)=\eta^{\prime \prime}} \mathcal{D} \eta(t) d^{2}\left(\sinh ^{2} \xi+\sin ^{2} \eta\right) \\
\times \exp \left\{\frac { \mathrm { i } } { \hbar } \int _ { t ^ { \prime } } ^ { t ^ { \prime \prime } } \left[\frac{M}{2}\left(d^{2}\left(\sinh ^{2} \xi+\sin ^{2} \eta\right)\left(\dot{\xi}^{2}+\dot{\eta}^{2}\right)+\dot{z}^{2}-\omega^{2} d^{2}\left(\cosh ^{2} \xi \cos ^{2} \eta+\sinh ^{2} \xi \sin ^{2} \eta\right)\right)\right.\right. \\
\left.\left.-F(z)-\frac{\hbar^{2}}{2 M d^{2}}\left(\frac{k_{1}^{2}-\frac{1}{4}}{\cosh ^{2} \xi \cos ^{2} \eta}+\frac{k_{2}^{2}-\frac{1}{4}}{\sinh ^{2} \xi \sin ^{2} \eta}\right)\right] d t\right\} .
\end{gathered}
$$

Except for the $z$-path integration, we can perform in the case of cartesian and circular polar polar the path integration explicitly yielding

$K^{\left(V_{2}\right)}\left(\vec{x}^{\prime \prime}, \vec{x}^{\prime} ; T\right)$

Cartesian Coordinates:

$$
\begin{aligned}
= & \left(\frac{M \omega}{\mathrm{i} \hbar \sin \omega T}\right)^{3} \prod_{i=1}^{2} \sqrt{x_{i}^{\prime} x_{i}^{\prime \prime}} \exp \left[-\frac{M \omega}{2 \mathrm{i} \hbar}\left(x_{i}^{\prime 2}+x_{i}^{\prime \prime 2}\right) \cot \omega T\right] I_{k_{i}}\left(\frac{M \omega x_{i}^{\prime} x_{i}^{\prime \prime}}{\mathrm{i} \hbar \sin \omega T}\right) \\
& \times \int_{z\left(t^{\prime}\right)=z^{\prime}}^{z\left(t^{\prime \prime}\right)=z^{\prime \prime}} \mathcal{D} z(t) \exp \left[\frac{\mathrm{i}}{\hbar} \int_{t^{\prime}}^{t^{\prime \prime}}\left(\frac{M}{2} \dot{z}^{2}-F(z)\right) d t\right] \\
= & \sum_{n_{1}, n_{2}=0}^{\infty} \mathrm{e}^{-\mathrm{i} E_{n_{1}, n_{2}} T / \hbar} \Psi_{n_{1}, n_{2}}\left(x_{1}^{\prime}, x_{2}^{\prime}\right) \Psi_{n_{1}, n_{2}}\left(x_{1}^{\prime \prime}, x_{2}^{\prime \prime}\right) \int d E_{\lambda_{z}} \mathrm{e}^{-\mathrm{i} E_{\lambda_{z}} T / \hbar} \Phi_{\lambda_{z}}^{*}\left(z^{\prime}\right) \Phi_{\lambda_{z}}\left(z^{\prime \prime}\right)
\end{aligned}
$$

Circular Polar Coordinates $\left.\left(\lambda_{1}=2 n \pm k_{1} \pm k_{2}+1\right)\right)$ :

$$
\begin{aligned}
& =\frac{M \omega}{\mathrm{i} \hbar \sin \omega T} \sum_{n=0}^{\infty} \Phi^{\left( \pm k_{2}, \pm k_{1}\right)}\left(\phi^{\prime}\right) \Phi^{\left( \pm k_{2}, \pm k_{1}\right)}\left(\phi^{\prime \prime}\right) \exp \left[-\frac{M \omega}{2 \mathrm{i} \hbar}\left(\rho^{\prime 2}+\rho^{\prime \prime 2}\right) \cot \omega T\right] I_{\lambda_{1}}\left(\frac{M \omega \rho^{\prime} \rho^{\prime \prime}}{\mathrm{i} \hbar \sin \omega T}\right) \\
& \times K_{F}\left(z^{\prime \prime}, z^{\prime} ; T\right)
\end{aligned}
$$


$=\sum_{n, m=0}^{\infty} \mathrm{e}^{-\mathrm{i} E_{n, m} T / \hbar} \Psi_{n, m}\left(\phi^{\prime}, \rho^{\prime}\right) \Psi_{n, m}\left(\phi^{\prime \prime}, \rho^{\prime \prime}\right) \int d E_{\lambda_{z}} \mathrm{e}^{-\mathrm{i} E_{\lambda_{z}} T / \hbar} \Phi_{\lambda_{z}}^{*}\left(z^{\prime}\right) \Phi_{\lambda_{z}}\left(z^{\prime \prime}\right)$

and in the sequel we denote the remaining $z$-path integral by

$$
K_{F}\left(z^{\prime \prime}, z^{\prime} ; T\right)=\int d E_{\lambda_{z}} \mathrm{e}^{-\mathrm{i} E_{\lambda_{z}} T / \hbar} \Phi_{\lambda_{z}}^{*}\left(z^{\prime}\right) \Phi_{\lambda_{z}}\left(z^{\prime \prime}\right)
$$

The wave-functions in $\left(x_{1}, x_{2}\right)$ and $(\rho, \phi)$ are the same as in 3.1.1.

3.3.3. We consider the potential $\left(k_{2}>0\right)$

$$
V_{3}(\vec{x})=\frac{M}{2} \omega^{2}\left(4 x^{2}+y^{2}\right)+\frac{\hbar^{2}}{2 M} \frac{k_{2}^{2}-\frac{1}{4}}{y^{2}}+F(z) .
$$

Due to the similarity of the corresponding two-dimensional case in 3.1.2 we just give the path integral formulations which have the form

$$
K^{\left(V_{3}\right)}\left(\vec{x}^{\prime \prime}, \vec{x}^{\prime} ; T\right)
$$

Cartesian Coordinates:

$$
\begin{aligned}
& =\int_{\vec{x}\left(t^{\prime}\right)=\vec{x}^{\prime}}^{\vec{x}\left(t^{\prime \prime}\right)=\vec{x}^{\prime \prime}} \mathcal{D} \vec{x}(t) \exp \left\{\frac{\mathrm{i}}{\hbar} \int_{t^{\prime}}^{t^{\prime \prime}}\left[\frac{M}{2}\left(\dot{\vec{x}}^{2}-\omega^{2}\left(4 x^{2}+y^{2}\right)\right)-F(z)-\frac{\hbar^{2}}{2 M} \frac{k_{2}^{2}-\frac{1}{4}}{y^{2}}\right] d t\right\} \\
& =\sum_{n, l=0}^{\infty} \mathrm{e}^{-\mathrm{i} E_{n, l} T / \hbar} \Psi_{n, l}\left(x^{\prime \prime}, y^{\prime \prime}\right) \Psi_{n, l}\left(x^{\prime}, y^{\prime}\right) \int d E_{\lambda_{z}} \mathrm{e}^{-\mathrm{i} E_{\lambda_{z}} T / \hbar} \Phi_{\lambda_{z}}^{*}\left(z^{\prime}\right) \Phi_{\lambda_{z}}\left(z^{\prime \prime}\right)
\end{aligned}
$$

Circular Parabolic Coordinates:

$$
\begin{aligned}
&= \int_{\eta\left(t^{\prime}\right)=\eta^{\prime}}^{\eta\left(t^{\prime \prime}\right)=\eta^{\prime \prime}} \mathcal{D} \eta(t) \int_{\xi\left(t^{\prime}\right)=\xi^{\prime}}^{\xi\left(t^{\prime \prime}\right)=\xi^{\prime \prime}} \mathcal{D} \xi(t)\left(\xi^{2}+\eta^{2}\right) \\
& \times \exp \left\{\frac{\mathrm{i}}{\hbar} \int_{t^{\prime}}^{t^{\prime \prime}}\left[\frac{M}{2}\left(\left(\xi^{2}+\eta^{2}\right)\left(\dot{\xi}^{2}+\dot{\eta}^{2}\right)-\omega^{2}\left(\left(\xi^{2}-\eta^{2}\right)^{2}-\xi^{2} \eta^{2}\right)\right)-\frac{k_{1}}{2}\left(\xi^{2}-\eta^{2}\right)-\hbar^{2} \frac{k_{2}^{2}-\frac{1}{4}}{2 M \eta^{2} \xi^{2}}\right] d t\right\} \\
& \times K_{F}\left(z^{\prime \prime}, z^{\prime} ; T\right),
\end{aligned}
$$

with the same Feynman kernel and wave-functions in $(x, y)$ as in 3.1.2.

3.3.4. We consider the potential $\left(k_{1,2}>0\right)$

$$
V_{4}(\vec{x})=-\frac{\alpha}{\sqrt{x^{2}+y^{2}}}+\frac{\hbar^{2}}{2 M}\left(\frac{k_{1}^{2} x}{y^{2} \sqrt{x^{2}+y^{2}}}+\frac{k_{2}^{2}-\frac{1}{4}}{y^{2}}\right)+F(z),
$$

We formulate the path integral in circular polar and circular parabolic coordinates, respectively

$$
\begin{aligned}
& K^{\left(V_{4}\right)}\left(\vec{x}^{\prime \prime}, \vec{x}^{\prime} ; T\right) \\
& =\int_{\vec{x}\left(t^{\prime}\right)=\vec{x}^{\prime}} \mathcal{D} \vec{x}(t) \exp \left\{\frac{\mathrm{i}}{\hbar} \int_{t^{\prime}}^{t^{\prime \prime}}\left[\frac{M}{2} \dot{\vec{x}}^{2}+\frac{\alpha}{\sqrt{x^{2}+y^{2}}}-\frac{\hbar^{2}}{2 M}\left(\frac{k_{1}^{2} x}{y^{2} \sqrt{x^{2}+y^{2}}}+\frac{k_{2}^{2}-\frac{1}{4}}{y^{2}}\right)-F(z)\right] d t\right\}
\end{aligned}
$$

Circular Polar Coordinates:

$$
=\int_{\rho\left(t^{\prime}\right)=\rho^{\prime}}^{\rho\left(t^{\prime \prime}\right)=\rho^{\prime \prime}} \rho \mathcal{D} \rho(t) \int_{\phi\left(t^{\prime}\right)=\phi^{\prime}}^{\phi\left(t^{\prime \prime}\right)=\phi^{\prime \prime}} \mathcal{D} \phi(t)
$$




$$
\begin{aligned}
& \times \exp \left\{\frac{\mathrm{i}}{\hbar} \int_{t^{\prime}}^{t^{\prime \prime}}\left[\frac{M}{2}\left(\dot{\rho}^{2}+\rho^{2} \dot{\phi}^{2}\right)+\frac{\alpha}{\rho}-\frac{\hbar^{2}}{2 M \rho^{2}}\left(\frac{k_{2}^{2}-k_{1}^{2}-\frac{1}{4}}{4 \cos ^{2} \frac{\phi}{2}}+\frac{k_{2}^{2}+k_{1}^{2}-\frac{1}{4}}{4 \sin ^{2} \frac{\phi}{2}}-\frac{1}{4}\right)\right] d t\right\} \\
& \times K_{F}\left(z^{\prime \prime}, z^{\prime} ; T\right)
\end{aligned}
$$

Circular Elliptic II Coordinates:

$$
\begin{gathered}
=\int_{\xi\left(t^{\prime}\right)=\xi^{\prime}}^{\xi\left(t^{\prime \prime}\right)=\xi^{\prime \prime}} \mathcal{D} \xi(t) \int_{\eta\left(t^{\prime}\right)=\eta^{\prime}}^{\eta\left(t^{\prime \prime}\right)=\eta^{\prime \prime}} \mathcal{D} \eta(t) d^{2}\left(\sinh ^{2} \xi+\sin ^{2} \eta\right) \int_{z\left(t^{\prime}\right)=z^{\prime}}^{z\left(t^{\prime \prime}\right)=z^{\prime \prime}} \mathcal{D} z(t) \\
\times \exp \left\{\frac { \mathrm { i } } { \hbar } \int _ { t ^ { \prime } } ^ { t ^ { \prime \prime } } \left[\frac{M}{2}\left(\left(\sinh ^{2} \xi^{2}+\sin ^{2} \eta\right)\left(\dot{\xi}^{2}+\dot{\eta}^{2}\right)+\dot{z}^{2}\right)+\frac{\alpha}{d(\cosh \xi+\cos \eta)}-F(z)\right.\right. \\
\left.\left.-\frac{\hbar^{2}}{2 M} \frac{1}{d^{2} \sinh ^{2} \xi \sin ^{2} \eta}\left(k_{1}^{2} \frac{\cosh \xi \cos \eta+1}{\cosh \xi+\cos \eta}+k_{2}^{2}-\frac{1}{2}\right)\right] d t\right\}
\end{gathered}
$$

Circular Parabolic Coordinates:

$$
\begin{aligned}
& =\int_{\eta\left(t^{\prime}\right)=\eta^{\prime}}^{\eta\left(t^{\prime \prime}\right)=\eta^{\prime \prime}} \mathcal{D} \eta(t) \int_{\xi\left(t^{\prime}\right)=\xi^{\prime}}^{\xi\left(t^{\prime \prime}\right)=\xi^{\prime \prime}} \mathcal{D} \xi(t)\left(\xi^{2}+\eta^{2}\right) \int_{z\left(t^{\prime}\right)=z^{\prime}}^{z\left(t^{\prime \prime}\right)=z^{\prime \prime}} \mathcal{D} z(t) \\
& \quad \times \exp \left\{\frac { \mathrm { i } } { \hbar } \int _ { t ^ { \prime } } ^ { t ^ { \prime \prime } } \left[\frac{M}{2}\left(\left(\xi^{2}+\eta^{2}\right)\left(\dot{\xi}^{2}+\dot{\eta}^{2}\right)+\dot{z}^{2}\right)+\frac{2 \alpha}{\xi^{2}+\eta^{2}}-F(z)\right.\right. \\
& \left.\left.\quad-\frac{\hbar^{2}}{2 M}\left(k_{1}^{2} \frac{\eta^{2}-\xi^{2}}{\xi^{2} \eta^{2}\left(\xi^{2}+\eta^{2}\right)}+\frac{k_{2}^{2}-\frac{1}{4}}{\xi^{2} \eta^{2}}\right)\right] d t\right\} .
\end{aligned}
$$

The circular polar an circular parabolic path integral formulations can be explicitly evaluated in terms of the corresponding Green function $\left(\lambda_{ \pm}^{2}=k_{2}^{2} \pm k_{1}^{2}, \lambda_{1}=n+\left(\lambda_{+}+\lambda_{-}+1\right) / 2\right.$, $\kappa=\alpha \sqrt{-M / 2 E} / \hbar)$

$K^{\left(V_{4}\right)}\left(\vec{x}^{\prime \prime}, \vec{x}^{\prime} ; T\right)$

Circular Polar Coordinates:

$$
\begin{aligned}
& =K_{F}\left(z^{\prime \prime}, z^{\prime} ; T\right)\left(\rho^{\prime} \rho^{\prime \prime}\right)^{-1 / 2} \frac{1}{2} \sum_{n=0}^{\infty} \Phi_{n}^{\left(\lambda_{+}, \lambda_{-}\right)}\left(\frac{\phi^{\prime}}{2}\right) \Phi_{n}^{\left(\lambda_{+}, \lambda_{-}\right)}\left(\frac{\phi^{\prime \prime}}{2}\right) \\
& \times \frac{1}{\hbar} \int_{\mathbb{R}} \frac{d E}{2 \pi \hbar} \mathrm{e}^{-\mathrm{i} E T / \hbar} \sqrt{-\frac{M}{2 E}} \frac{\Gamma\left(\frac{1}{2}+\lambda_{1}-\kappa\right)}{\Gamma\left(1+2 \lambda_{1}\right)} W_{\kappa, \lambda_{1}}\left(\sqrt{-8 M E} \frac{\rho_{>}}{\hbar}\right) M_{\kappa, \lambda_{1}}\left(\sqrt{-8 M E} \frac{\rho_{<}}{\hbar}\right) \\
& =\int d E_{\lambda_{z}} \mathrm{e}^{-\mathrm{i} E_{\lambda_{z}} T / \hbar} \Phi_{\lambda_{z}}^{*}\left(z^{\prime}\right) \Phi_{\lambda_{z}}\left(z^{\prime \prime}\right) \\
& \times \sum_{m=-0}^{\infty}\left\{\sum_{n=0}^{\infty} \frac{\Phi_{\lambda_{1}, m}^{*}\left(\rho^{\prime}, \phi^{\prime}\right) \Phi_{\lambda_{1}, m}\left(\rho^{\prime \prime}, \phi^{\prime \prime}\right)}{E_{n}-E}+\int_{\mathbb{R}} d p \frac{\Phi_{\lambda_{z}, p}^{*}\left(\rho^{\prime}, \phi^{\prime}\right) \Phi_{\lambda, p}^{\alpha)}\left(\rho^{\prime \prime}, \phi^{\prime \prime}\right)}{\hbar^{2} p^{2} / 2 M-E}\right\}
\end{aligned}
$$

Circular Parabolic Coordinates:

$$
\begin{aligned}
=\int d E_{\lambda_{z}} \mathrm{e}^{-\mathrm{i} E_{\lambda_{z}} T / \hbar} \Phi_{\lambda_{z}}^{*}\left(z^{\prime}\right) \Phi_{\lambda_{z}}\left(z^{\prime \prime}\right) \\
\quad \times\left\{\sum_{n_{1}, n_{2}=0}^{\infty} \frac{\Psi_{n_{1}, n_{2}}^{*}\left(\xi^{\prime}, \eta^{\prime}\right) \Psi_{n_{1}, n_{2}}\left(\xi^{\prime \prime}, \eta^{\prime \prime}\right)}{E_{n_{1}, n_{2}}-E}+\int_{0}^{\infty} d p \int_{\mathbb{R}} d \zeta \frac{\Psi_{p, \zeta}^{*}\left(\xi^{\prime}, \eta^{\prime}\right) \Psi_{p, \zeta}\left(\xi^{\prime \prime}, \eta^{\prime \prime}\right)}{\hbar^{2} p^{2} / 2 M-E}\right\},
\end{aligned}
$$

with the same wave-functions in $(\phi, \rho)$ and $(\xi, \eta)$ as in 3.2 .3 .

3.3.5. The next three potential involve an arbitrary $\phi$-dependence where $F(y / x)=\gamma^{2}>0$ describes the well-known double ring-shaped oscillator (c.f. e.g. Carpio-Bernido et al. [12, 13], Kibler et al. [76, 77, 81, Lutsenko et al. [91], and Quesne [116]). We consider the potential 
$\left(k_{3}>0\right)$

$$
V_{5}(\vec{x})=\frac{M}{2} \omega^{2}\left(x^{2}+y^{2}+z^{2}\right)+\frac{\hbar^{2}}{2 M}\left(\frac{k_{3}^{2}-\frac{1}{4}}{z^{2}}+\frac{F(y / x)}{x^{2}+y^{2}}\right) .
$$

We obtain the following path integral formulations in the corresponding separating coordinate systems

$$
K^{\left(V_{5}\right)}\left(\vec{x}^{\prime \prime}, \vec{x}^{\prime} ; T\right)
$$

Spherical Coordinates:

$$
\begin{aligned}
& \int_{r\left(t^{\prime}\right)=r^{\prime}}^{r\left(t^{\prime \prime}\right)=r^{\prime \prime}} r^{2} \mathcal{D} r(t) \int_{\theta\left(t^{\prime}\right)=\theta^{\prime}}^{\theta\left(t^{\prime \prime}\right)=\theta^{\prime \prime}} \sin \theta \mathcal{D} \theta(t) \int_{\phi\left(t^{\prime}\right)=\phi^{\prime}}^{\phi\left(t^{\prime \prime}\right)=\phi^{\prime \prime}} \mathcal{D} \phi(t) \\
& \times \exp \left\{\frac { \mathrm { i } } { \hbar } \int _ { t ^ { \prime } } ^ { t ^ { \prime \prime } } \left[\frac{M}{2}\left(\dot{r}^{2}+r^{2} \dot{\theta}^{2}+r^{2} \sin ^{2} \theta \dot{\phi}^{2}-\omega^{2} r^{2}\right)\right.\right. \\
& \left.\left.-\frac{\hbar^{2}}{2 M r^{2}}\left(\frac{F(\tan \phi)-\frac{1}{4}}{\sin ^{2} \theta}+\frac{k_{3}^{2}-\frac{1}{4}}{\cos ^{2} \theta}-\frac{1}{4}\right)\right] d t\right\}
\end{aligned}
$$

Circular Polar Coordinates:

$$
\begin{aligned}
& =\int_{z\left(t^{\prime}\right)=z^{\prime}}^{z\left(t^{\prime \prime}\right)=z^{\prime \prime}} \mathcal{D} z(t) \int_{\rho\left(t^{\prime}\right)=\rho^{\prime}}^{\rho\left(t^{\prime \prime}\right)=\rho^{\prime \prime}} \rho \mathcal{D} \rho(t) \int_{\phi\left(t^{\prime}\right)=\phi^{\prime}}^{\phi\left(t^{\prime \prime}\right)=\phi^{\prime \prime}} \mathcal{D} \phi(t) \\
& \quad \times \exp \left\{\frac{\mathrm{i}}{\hbar} \int_{t^{\prime}}^{t^{\prime \prime}}\left[\frac{M}{2}\left(\dot{\rho}^{2}+\rho^{2} \dot{\phi}^{2}+\dot{z}^{2}-\omega^{2}\left(\rho^{2}+z^{2}\right)\right)-\frac{\hbar^{2}}{2 M}\left(\frac{F(\tan \phi)-\frac{1}{4}}{\rho^{2}}+\frac{k_{3}^{2}-\frac{1}{4}}{z^{2}}\right)\right] d t\right\}
\end{aligned}
$$

Oblate Spheroidal Coordinates:

$$
\begin{gathered}
\overline{\bar{\mu}\left(t^{\prime \prime}\right)=\bar{\mu}^{\prime \prime}} \mathcal{D} \bar{\mu}(t) \int_{\bar{\mu}\left(t^{\prime}\right)=\bar{\mu}^{\prime}}^{\bar{\nu}\left(t^{\prime \prime}\right)=\bar{\nu}^{\prime \prime}} \mathcal{D} \bar{\nu}(t) \bar{d}^{3}\left(\cosh ^{2} \bar{\mu}-\sin ^{2} \bar{\nu}\right) \cosh \bar{\mu} \sin \bar{\nu} \int_{\phi\left(t^{\prime}\right)=\phi^{\prime}}^{\phi\left(t^{\prime \prime}\right)=\bar{\nu}^{\prime}} \mathcal{D} \phi(t) \\
\times \exp \left\{\frac { \mathrm { i } } { \overline { \hbar } } \int _ { t ^ { \prime } } ^ { t ^ { \prime \prime } } \left[\frac { M } { 2 } \overline { d } ^ { 2 } \left(\left(\cosh ^{2} \bar{\mu}-\sin ^{2} \bar{\nu}\right)\left(\dot{\bar{\mu}}^{2}+\dot{\bar{\nu}}^{2}\right)+\cosh ^{2} \bar{\mu} \sin ^{2} \bar{\nu} \dot{\phi}^{2}\right.\right.\right. \\
\left.-\omega^{2}\left(\cos ^{2} \bar{\mu} \sin ^{2} \bar{\nu}+\sinh ^{2} \bar{\mu} \cos ^{2} \bar{\nu}\right)\right) \\
\left.\left.-\frac{\hbar^{2}}{2 M \bar{d}^{2}}\left(\frac{F(\tan \phi)-\frac{1}{4}}{\cosh ^{2} \bar{\mu} \sin ^{2} \bar{\nu}}+\frac{k_{3}^{2}-\frac{1}{4}}{\sinh ^{2} \bar{\mu} \cos ^{2} \bar{\nu}}\right)\right] d t\right\}
\end{gathered}
$$

Prolate Spheroidal Coordinates:

$$
\begin{gathered}
=\int_{\mu\left(t^{\prime}\right)=\mu^{\prime}}^{\mu\left(t^{\prime \prime}\right)=\mu^{\prime \prime}} \mathcal{D} \mu(t) \int_{\nu\left(t^{\prime}\right)=\nu^{\prime}}^{\nu\left(t^{\prime \prime}\right)=\nu^{\prime \prime}} \mathcal{D} \nu(t) d^{3}\left(\sinh ^{2} \mu+\sin ^{2} \nu\right) \sinh \mu \sin \nu \int_{\phi\left(t^{\prime}\right)=\phi^{\prime}}^{\phi\left(t^{\prime \prime}\right)=\phi^{\prime \prime}} \mathcal{D} \phi(t) \\
\times \exp \left\{\frac { \mathrm { i } } { \hbar } \int _ { t ^ { \prime } } ^ { t ^ { \prime \prime } } \left[\frac { M } { 2 } d ^ { 2 } \left(\left(\sinh ^{2} \mu+\sin ^{2} \nu\right)\left(\dot{\mu}^{2}+\dot{\nu}^{2}\right)+\sinh ^{2} \mu \sin ^{2} \nu \dot{\phi}^{2}\right.\right.\right. \\
\left.-\omega^{2}\left(\sinh ^{2} \mu \sin ^{2} \nu+\cosh ^{2} \mu \cos ^{2} \nu\right)\right) \\
\left.\left.-\frac{\hbar^{2}}{2 M d^{2}}\left(\frac{F(\tan \phi)-\frac{1}{4}}{\sinh ^{2} \mu \sin ^{2} \nu}+\frac{k_{3}^{2}-\frac{1}{4}}{\cosh ^{2} \mu \cos ^{2} \nu}\right)\right] d t\right\} .
\end{gathered}
$$

We consider the spherical and circular polar coordinate cases, the others can be treated in a similar way as far as the separation of the $\phi$-path integration is concerned. We denote the 
energy-spectrum of the $\phi$-dependent problem by $E_{\lambda_{\phi}}=\hbar^{2} \lambda_{\phi}^{2} / 2 M$. The most important case occurs when $F(\tan \phi)=$ constant. Then the quantum motion in $\phi$ are just circular waves and we have in this case $\Phi_{\lambda_{\phi}}(\phi) \equiv \Phi_{\nu}(\phi)=\mathrm{e}^{\mathrm{i} \nu \phi} / \sqrt{2 \pi}(\nu \in \mathbb{Z})$. This is also the case for the potentials $V_{6}$ and $V_{7}$. We have $\left(\lambda_{2}=2 n+\lambda_{\phi} \pm k_{3}+1\right)$ 
$K^{\left(V_{5}\right)}\left(\vec{x}^{\prime \prime}, \vec{x}^{\prime} ; T\right)$

Spherical Coordinates [10]:

$$
\begin{aligned}
= & \left(r^{\prime} r^{\prime \prime} \sin \theta^{\prime} \sin \theta^{\prime \prime}\right)^{-1 / 2} \int d E_{\lambda_{\phi}} \Phi_{\lambda_{\phi}}^{*}\left(\phi^{\prime}\right) \Phi_{\lambda_{\phi}}\left(\phi^{\prime \prime}\right) \\
& \times \sum_{n=0}^{\infty} \Phi_{n}^{\left(\lambda_{\phi}, \pm k_{3}\right)}\left(\theta^{\prime}\right) \Phi_{n}^{\left(\lambda_{\phi}, \pm k_{3}\right)}\left(\theta^{\prime \prime}\right) \frac{M \omega}{\mathrm{i} \hbar \sin \omega T} \exp \left[\frac{\mathrm{i} M}{2 \hbar}\left(r^{\prime 2}+r^{\prime \prime 2}\right) \cot \omega T\right] I_{\lambda_{2}}\left(\frac{M \omega r^{\prime} r^{\prime \prime}}{\mathrm{i} \hbar \sin \omega T}\right) \\
= & \int d E_{\lambda_{\phi}} \sum_{n, l=0}^{\infty} \mathrm{e}^{-\mathrm{i} E_{N} T / \hbar} \Psi_{\lambda_{\phi}, n, l}^{*}\left(\phi^{\prime}, \theta^{\prime}, r^{\prime}\right) \Psi_{\lambda_{\phi}, n, l}\left(\phi^{\prime \prime}, \theta^{\prime \prime}, r^{\prime \prime}\right)
\end{aligned}
$$

Circular Polar Coordinates:

$$
\begin{aligned}
& =\left(\frac{M \omega}{\mathrm{i} \hbar \sin \omega T}\right)^{2} \sqrt{z^{\prime} z^{\prime \prime}} \exp \left[-\frac{M \omega}{2 \mathrm{i} \hbar}\left(z^{\prime 2}+z^{\prime \prime 2}+\rho^{\prime 2}+\rho^{\prime \prime 2}\right) \cot \omega T\right] I_{ \pm k_{3}}\left(\frac{M \omega z^{\prime} z^{\prime \prime}}{\mathrm{i} \hbar \sin \omega T}\right) \\
& \quad \times \int d E_{\lambda_{\phi}} \Phi_{\lambda_{\phi}}^{*}\left(\phi^{\prime}\right) \Phi_{\lambda_{\phi}}\left(\phi^{\prime \prime}\right) I_{\lambda_{\phi}}\left(\frac{M \omega \rho^{\prime} \rho^{\prime \prime}}{\mathrm{i} \hbar \sin \omega T}\right) \\
& =\int d E_{\lambda_{\phi}} \sum_{m, n_{z}=0}^{\infty} \mathrm{e}^{-\mathrm{i} E_{N} T / \hbar} \Psi_{\lambda_{\phi}, m, n_{z}}^{*}\left(\phi^{\prime}, \rho^{\prime}, z^{\prime}\right) \Phi_{\lambda_{\phi}, m, n_{z}}\left(\phi^{\prime \prime}, \rho^{\prime \prime}, z^{\prime \prime}\right) .
\end{aligned}
$$

The wave-functions and energy-levels are given by

Spherical Coordinates:

$$
\begin{aligned}
& \Phi_{\lambda_{\phi}, n, l}(\phi, \theta, r)=(r \sin \theta)^{-1 / 2} \Phi_{\lambda_{\phi}}(\phi) \Phi_{m}^{\left(\lambda_{\phi}, \pm k_{3}\right)}\left(\theta^{\prime}\right) \\
& \quad \times \sqrt{\frac{2 M \omega}{\hbar}\left(\frac{M \omega}{\hbar}\right)^{\lambda_{2} / 2} \sqrt{\frac{l !}{\Gamma\left(l+\lambda_{2}+1\right)}} r^{\lambda_{2}+1 / 2} \exp \left(-\frac{M \omega}{2 \hbar} r^{2}\right) L_{l}^{\left(\lambda_{2}\right)}\left(\frac{M \omega}{\hbar} r^{2}\right),} \\
& E_{N}=\hbar \omega\left(N \pm k_{3}+2\right), \quad N=2 l+2 n+\lambda_{\phi},
\end{aligned}
$$

Circular Polar Coordinates:

$$
\begin{aligned}
& \Psi_{\lambda_{\phi}, m, n_{z}}(\phi, \rho, z)=\Phi_{\lambda_{\phi}}(\phi) \frac{2 M \omega}{\hbar}\left(\frac{M \omega}{\hbar}\right)^{ \pm k_{3} / 2} \\
& \quad \times \sqrt{\frac{n_{z} !}{\Gamma\left(n_{z} \pm k_{3}+1\right)}} z^{1 / 2 \pm k_{3}} \exp \left(-\frac{M \omega}{2 \hbar} z^{2}\right) L_{n_{z}}^{\left( \pm k_{3}\right)}\left(\frac{M \omega}{\hbar} z^{2}\right) \\
& \quad \times\left(\frac{M \omega}{\hbar} \rho^{2}\right)^{\lambda_{\phi} / 2} \sqrt{\frac{m !}{\Gamma\left(m+\lambda_{\phi}+1\right)}} \exp \left(-\frac{M \omega}{2 \hbar} \rho^{2}\right) L_{m}^{\left(\lambda_{\phi}\right)}\left(\frac{M \omega}{\hbar} \rho^{2}\right), \\
& E_{N}=\hbar \omega\left(N \pm k_{3}+2\right), \quad N=2 m+2 n_{z}+\lambda_{\phi} .
\end{aligned}
$$

For $F(\tan \phi)=$ constant, $\lambda_{\phi}=|\nu|$, c.f. also $V_{6}$ and $V_{7}$.

\subsubsection{We consider the potential}

$$
V_{6}(\vec{x})=\frac{M}{2} \omega^{2}\left(x^{2}+y^{2}+4 z^{2}\right)+\frac{\hbar^{2}}{2 M} \frac{F(y / x)}{x^{2}+y^{2}} .
$$

We obtain the following path integral formulations in the corresponding separable coordinate systems We write down the corresponding path integral formulations

$$
K^{\left(V_{6}\right)}\left(\vec{x}^{\prime \prime}, \vec{x}^{\prime} ; T\right)
$$

Circular Polar Coordinates:

$$
=\int_{z\left(t^{\prime}\right)=z^{\prime}}^{z\left(t^{\prime \prime}\right)=z^{\prime \prime}} \mathcal{D} z(t) \int_{\rho\left(t^{\prime}\right)=\rho^{\prime}}^{\rho\left(t^{\prime \prime}\right)=\rho^{\prime \prime}} \rho \mathcal{D} \rho(t) \int_{\phi\left(t^{\prime}\right)=\phi^{\prime}}^{\phi\left(t^{\prime \prime}\right)=\phi^{\prime \prime}} \mathcal{D} \phi(t)
$$




$$
\begin{aligned}
& \quad \times \exp \left\{\frac{\mathrm{i}}{\hbar} \int_{t^{\prime}}^{t^{\prime \prime}}\left[\frac{M}{2}\left(\dot{\rho}^{2}+\rho^{2} \dot{\phi}^{2}+\dot{z}^{2}-\omega^{2}\left(\rho^{2}+4 z^{2}\right)\right)-\hbar^{2} \frac{F(\tan \phi)-\frac{1}{4}}{2 M \rho^{2}}\right] d t\right\} \\
& =\left(\rho^{\prime} \rho^{\prime \prime}\right)^{-1 / 2} \int d E_{\lambda_{\phi}} \Phi_{\lambda_{\phi}}^{*}\left(\phi^{\prime}\right) \Phi_{\lambda_{\phi}}\left(\phi^{\prime \prime}\right) \\
& \quad \times \int_{z\left(t^{\prime}\right)=z^{\prime}} \mathcal{D} z(t) \int_{\rho\left(t^{\prime}\right)=\rho^{\prime}} \mu_{\lambda_{\phi}}\left[\rho^{2}\right] \mathcal{D} \rho(t) \exp \left[\frac{\mathrm{i} M}{2 \hbar} \int_{t^{\prime}}^{t^{\prime \prime}}\left(\dot{\rho}^{2}+\dot{z}^{2}-\omega^{2}\left(\rho^{2}+4 z^{2}\right)\right) d t\right] \\
& =\int d E_{\lambda_{\phi}} \sum_{n_{z}, l=0}^{\infty} \mathrm{e}^{-\mathrm{i} E_{n_{z}, m} T / \hbar} \Psi_{n_{z}, \lambda_{\phi}, m}^{*}\left(\phi^{\prime}, \rho^{\prime}, z^{\prime}\right) \Psi_{n_{z}, \lambda_{\phi}, m}\left(\phi^{\prime \prime}, \rho^{\prime \prime}, z^{\prime \prime}\right)
\end{aligned}
$$

Parabolic Coordinates:

$$
\begin{aligned}
& =\left(\xi^{\prime} \xi^{\prime \prime} \eta^{\prime} \eta^{\prime \prime}\right)^{-1 / 2} \int d E_{\lambda_{\phi}} \Phi_{\lambda_{\phi}}^{*}\left(\phi^{\prime}\right) \Phi_{\lambda_{\phi}}\left(\phi^{\prime \prime}\right) \\
& \quad \times \int_{\mathbb{R}} \frac{d E}{2 \pi \hbar} \mathrm{e}^{-\mathrm{i} E T / \hbar} \int_{0}^{\infty} d s^{\prime \prime} \int_{\eta(0)=\eta^{\prime}}^{\eta\left(s^{\prime \prime}\right)=\eta^{\prime \prime}} \mathcal{D} \eta(s) \int_{\xi(0)=\xi^{\prime}}^{\xi\left(s^{\prime \prime}\right)=\xi^{\prime \prime}} \mathcal{D} \xi(s) \\
& \quad \times \exp \left\{\frac{\mathrm{i}}{\hbar} \int_{0}^{s^{\prime \prime}}\left[\frac{M}{2}\left(\left(\dot{\xi}^{2}+\dot{\eta}^{2}\right)-\omega^{2}\left(\xi^{6}+\eta^{6}\right)\right)+E\left(\xi^{2}+\eta^{2}\right)-\hbar^{2} \frac{\lambda_{\phi}^{2}-\frac{1}{4}}{2 M}\left(\frac{1}{\xi^{2}}+\frac{1}{\eta^{2}}\right)\right] d s\right\} .
\end{aligned}
$$

Here we have used the same notation as in the previous case. The wave-functions and the energy-spectrum in circular polar coordinates are given by

$$
\begin{aligned}
& \Psi_{n_{z}, \lambda_{\phi}, m}(\phi, \rho, z)=\Phi_{\lambda_{\phi}}(\phi) \sqrt{\frac{2 M \omega}{\hbar}}\left(\frac{M \omega}{\hbar} \rho^{2}\right)^{\lambda_{\phi} / 2} \sqrt{\frac{m !}{\Gamma\left(m+\lambda_{\phi}+1\right)}} \exp \left(-\frac{M \omega}{2 \hbar} \rho^{2}\right) L_{m}^{\left(\lambda_{\phi}\right)}\left(\frac{M \omega}{\hbar} \rho^{2}\right) \\
& \times \sqrt{\sqrt{\frac{2 M \omega}{\pi \hbar}} \frac{1}{2^{n_{z}} n_{z} !}} \exp \left(-\frac{M \omega}{\hbar} z^{2}\right) H_{n_{z}}\left(\sqrt{\frac{2 M \omega}{\hbar}} z\right), \\
& E_{n_{z}, m}=\hbar \omega\left(2 m+\lambda_{\phi}+n_{z}+\frac{3}{2}\right) \text {. }
\end{aligned}
$$

3.3.7. We consider $\left(k_{1}>0\right)$

$$
V_{7}(\vec{x})=-\frac{\alpha}{\sqrt{x^{2}+y^{2}+z^{2}}}+\frac{\hbar^{2}}{2 M\left(x^{2}+y^{2}\right)}\left(\frac{k_{1}^{2} z}{\sqrt{x^{2}+y^{2}+y^{2}}}+F\left(\frac{y}{x}\right)\right) .
$$

For $F(y / x)=\gamma^{2}>0$ this potential is known as the ring-shaped Hartmann potential (c.f. Calogero [9], Carpio-Bernido et al. [10, 11, 13, 12], Chetouani at al. [17], Gerry [46], Granovsky et al. [50], Grosche [56], Guha and Mukherjee [62], Hartmann [63], Kibler et al. [76]- [79], [81], Lutsenko et al. [90, Vaidya and Boschi-Filho [125], and Zhedanov [131]; compare also the connection with a Coulomb plus Aharonov-Bohm solenoid, e.g. Chetouani et al. [18], Kibler and Negadi [80], and Sökmen [117]). We write down the corresponding path integral formulations where only the corresponding Green function can be evaluated $\left[\lambda_{ \pm}^{2}=\lambda_{\phi}^{2} \pm k_{1}^{2}, \lambda_{1}=n+\left(\lambda_{+}+\right.\right.$ $\left.\left.\lambda_{-}+1\right) / 2, \kappa=\alpha \sqrt{-M / 2 E} / \hbar\right]$

$$
\frac{\mathrm{i}}{\hbar} \int_{0}^{\infty} d T \mathrm{e}^{\mathrm{i} E T / \hbar} K^{\left(V_{7}\right)}\left(\vec{x}^{\prime \prime}, \vec{x}^{\prime} ; T\right)
$$

Spherical Coordinates:

$$
=\frac{\mathrm{i}}{\hbar} \int_{0}^{\infty} d T \mathrm{e}^{\mathrm{i} E T / \hbar} \int_{r\left(t^{\prime}\right)=r^{\prime}}^{r\left(t^{\prime \prime}\right)=r^{\prime \prime}} r^{2} \mathcal{D} r(t) \int_{\theta\left(t^{\prime}\right)=\theta^{\prime}}^{\theta\left(t^{\prime \prime}\right)=\theta^{\prime \prime}} \sin \theta \mathcal{D} \theta(t) \int_{\phi\left(t^{\prime}\right)=\phi^{\prime}}^{\phi\left(t^{\prime \prime}\right)=\phi^{\prime \prime}} \mathcal{D} \phi(t)
$$




$$
\begin{aligned}
& \times \exp \left\{\frac { \mathrm { i } } { \hbar } \int _ { t ^ { \prime } } ^ { t ^ { \prime \prime } } \left[\frac{M}{2}\left(\dot{r}^{2}+r^{2} \dot{\theta}^{2}+r^{2} \sin ^{2} \theta \dot{\phi}^{2}\right)\right.\right. \\
& \left.\left.\quad+\frac{\alpha}{r}-\frac{\hbar^{2}}{2 M r^{2}}\left(\frac{k_{1}^{2} \cos \theta}{\sin ^{2} \theta}+\frac{F(\tan \phi)-\frac{1}{4}}{\sin ^{2} \theta}-\frac{1}{4}\right)\right] d t\right\} \\
& =\left(r^{\prime 2} r^{\prime \prime 2} \sin \theta^{\prime} \sin \theta^{\prime \prime}\right)^{-1 / 2} \int d E_{\lambda_{\phi}} \Phi_{\lambda_{\phi}}^{*}\left(\phi^{\prime}\right) \Phi_{\lambda_{\phi}}\left(\phi^{\prime \prime}\right) \frac{1}{2} \sum_{n=0}^{\infty} \Phi_{n}^{\left(\lambda_{+}, \lambda_{-}\right)}\left(\frac{\theta^{\prime}}{2}\right) \Phi_{n}^{\left(\lambda_{+}, \lambda_{-}\right)}\left(\frac{\theta^{\prime \prime}}{2}\right) \\
& \quad \times \frac{1}{\hbar} \sqrt{-\frac{M}{2 E}} \frac{\Gamma\left(\frac{1}{2}+\lambda_{1}-\kappa\right)}{\Gamma\left(2 \lambda_{2}+1\right)} W_{\kappa, \lambda_{1}}\left(\sqrt{-8 M E} \frac{r_{>}}{\hbar}\right) M_{\kappa, \lambda_{1}}\left(\sqrt{-8 M E} \frac{r_{<}}{\hbar}\right) \\
& =\sum_{n, m=0}^{\infty}\left\{\sum_{l=0}^{\infty} \frac{\Psi_{n, m, l}^{*}\left(\theta^{\prime}, \phi^{\prime}, r^{\prime}\right) \Psi_{n, m, l}\left(\theta^{\prime \prime}, \phi^{\prime \prime}, r^{\prime \prime}\right)}{E_{N}-E}+\int_{\mathbb{R}} d p \frac{\Psi_{n, m, p}^{*}\left(\theta^{\prime}, \phi^{\prime}, r^{\prime}\right) \Psi_{n, m, p}\left(\theta^{\prime \prime}, \phi^{\prime \prime}, r^{\prime \prime}\right)}{\hbar^{2} p^{2} / 2 M-E}\right\}
\end{aligned}
$$

Parabolic Coordinates:

$$
\begin{aligned}
& =\frac{\mathrm{i}}{\hbar} \int_{0}^{\infty} d T \mathrm{e}^{\mathrm{i} E T / \hbar} \int_{\eta\left(t^{\prime}\right)=\eta^{\prime}}^{\eta\left(t^{\prime \prime}\right)=\eta^{\prime \prime}} \mathcal{D} \eta(t) \int_{\xi\left(t^{\prime}\right)=\xi^{\prime}}^{\xi\left(t^{\prime \prime}\right)=\xi^{\prime \prime}} \mathcal{D} \xi(t)\left(\xi^{2}+\eta^{2}\right) \xi \eta \int_{\phi\left(t^{\prime}\right)=\phi^{\prime}}^{\phi\left(t^{\prime \prime}\right)=\phi^{\prime \prime}} \mathcal{D} \phi(t) \\
& \quad \times \exp \left\{\frac { \mathrm { i } } { \hbar } \int _ { t ^ { \prime } } ^ { t ^ { \prime \prime } } \left[\frac{M}{2}\left(\left(\xi^{2}+\eta^{2}\right)\left(\dot{\xi}^{2}+\dot{\eta}^{2}\right)+\xi^{2} \eta^{2} \dot{\phi}^{2}\right)\right.\right. \\
& \left.\left.\quad+\frac{2 \alpha}{\xi^{2}+\eta^{2}}-\frac{\hbar^{2}}{2 M \xi^{2} \eta^{2}}\left(k_{1}^{2} \frac{\eta^{2}-\xi^{2}}{\eta^{2}+\xi^{2}}+F(\tan \phi)-\frac{1}{4}\right)\right] d t\right\} \\
& =\sum_{n=0}^{\infty}\left[\sum_{n_{1}, n_{2}=0}^{\infty} \frac{\Psi_{n, n_{1}, n_{2}}\left(\phi^{\prime}, \xi^{\prime}, \eta^{\prime}\right) \Psi_{n, n_{1}, n_{2}}\left(\phi^{\prime \prime}, \xi^{\prime \prime}, \eta^{\prime \prime}\right)}{E_{n_{1}, n_{2}}-E}\right. \\
& \left.+\int_{0}^{\infty} d p \int_{\mathbb{R}} d \zeta \frac{\Psi_{n, p, \zeta}^{*}\left(\phi^{\prime}, \xi^{\prime}, \eta^{\prime}\right) \Psi_{n, p, \zeta}\left(\phi^{\prime \prime}, \xi^{\prime \prime}, \eta^{\prime \prime}\right)}{\hbar^{2} p^{2} / 2 M-E}\right] .
\end{aligned}
$$

The bound state wave-functions in the polar coordinates are

$$
\begin{gathered}
\Psi_{n, m, l}(\theta, \phi, r)=(2 \sin \theta)^{-1 / 2} \Psi_{\lambda_{\phi}}^{*}(\phi) \Phi_{m}^{\left(\lambda_{+}, \lambda_{-}\right)}\left(\frac{\theta}{2}\right) \\
\quad \times \frac{2}{\left(l+\lambda_{1}+\frac{1}{2}\right)^{2}}\left[\frac{l !}{a^{3}\left(l+\lambda_{1}+\frac{1}{2}\right) \Gamma\left(l+2 \lambda_{1}+1\right)}\right]^{1 / 2} \\
\quad \times\left(\frac{2 r}{a\left(l+\lambda_{1}+\frac{1}{2}\right)}\right)^{\lambda_{1}} \exp \left(-\frac{r}{a\left(l+\lambda_{1}+\frac{1}{2}\right)}\right) L_{l}^{\left(2 \lambda_{1}\right)}\left(\frac{2 r}{a\left(l+\lambda_{1}+\frac{1}{2}\right)}\right), \\
E_{N}=-\frac{M \alpha^{2}}{\hbar^{2} N^{2}}, \quad N=l+n+\frac{1}{2}\left(1+\lambda_{-}+\lambda_{+}\right) .
\end{gathered}
$$

The continuous wave-functions are

$$
\begin{aligned}
\Psi_{n, l, p}(\theta, \phi, r)==(2 & \sin \theta)^{-1 / 2} \Phi_{\lambda_{\phi}}(\phi) \Phi_{n}^{\left(\lambda_{+}, \lambda_{-}\right)}\left(\frac{\theta}{2}\right) \\
& \times \frac{\Gamma\left(\frac{1}{2}+\lambda_{2}-\mathrm{i} / a p\right)}{\sqrt{2 \pi} r \Gamma\left(2 \lambda_{2}+1\right)} \exp \left(\frac{\pi}{2 a p}\right) M_{\mathrm{i} / a p, \lambda_{2}}(-2 \mathrm{i} p r) .
\end{aligned}
$$

The wave-functions in parabolic coordinates are the same as in 3.2.3, except for the modification that $\lambda_{ \pm}^{2}=\lambda_{\phi}^{2} \pm k_{1}^{2}$ instead of $\lambda_{ \pm}^{2}=k_{2} \pm k_{1}^{2}$. 
The potential $V_{7}$ is also separable in prolate spheroidal II coordinates and we have the identity

$$
\begin{aligned}
& K^{\left(V_{7}\right)}\left(\vec{x}^{\prime \prime}, \vec{x}^{\prime} ; T\right) \\
& =\int_{\mu\left(t^{\prime}\right)=\mu^{\prime}}^{\mu\left(t^{\prime \prime}\right)=\mu^{\prime \prime}} \mathcal{D} \mu(t) \int_{\nu\left(t^{\prime}\right)=\nu^{\prime}}^{\nu\left(t^{\prime \prime}\right)=\nu^{\prime \prime}} \mathcal{D} \nu(t) d^{3}\left(\sinh ^{2} \mu+\sin ^{2} \nu\right) \sin \nu \sinh \mu \int_{\phi\left(t^{\prime}\right)=\phi^{\prime}}^{\phi\left(t^{\prime \prime}\right)=\phi^{\prime \prime}} \mathcal{D} \phi(t) \\
& \quad \times \exp \left\{\frac { \mathrm { i } } { \hbar } \int _ { t ^ { \prime } } ^ { t ^ { \prime \prime } } \left[\frac{M}{2} d^{2}\left(\left(\sinh ^{2} \mu+\sin ^{2} \nu\right)\left(\dot{\mu}^{2}+\dot{\nu}^{2}\right)+\sinh ^{2} \mu \sin ^{2} \nu \dot{\phi}^{2}\right)\right.\right. \\
& \left.\left.\quad+\frac{\hbar^{2}}{d(\cosh \mu+\cos \nu)}-\frac{\hbar_{1}^{2}(\cosh \mu \cos \nu+1)}{2 M d^{2} \sinh ^{2} \mu \sin ^{2} \nu}\left(\frac{\cosh \mu+\cos \nu}{\alpha}+F(\tan \phi)-\frac{1}{4}\right)\right] d t\right\} \\
& =\left(d^{2} \sinh \mu^{\prime} \sinh \mu^{\prime \prime} \sin \nu^{\prime} \sin \nu^{\prime \prime}\right)^{-1 / 2} \int d E_{\lambda_{\phi}} \Phi_{\lambda_{\phi}}^{*}\left(\phi^{\prime}\right) \Phi_{\lambda_{\phi}}\left(\phi^{\prime \prime}\right) \int_{\mathbb{R}} \frac{d E}{2 \pi \hbar} \mathrm{e}^{-\mathrm{i} E T / \hbar \int_{0}^{\infty}} d s^{\prime \prime} \\
& \quad \int_{\mu\left(s^{\prime \prime}\right)=\mu^{\prime \prime}} \mathcal{D} \mu(s) \int_{\nu\left(s^{\prime \prime}\right)=\nu^{\prime \prime}} \mathcal{D} \nu(s) \exp \left\{\frac { \mathrm { i } } { \hbar } \int _ { 0 } ^ { s ^ { \prime \prime } } \left[\frac{M}{2}\left(\dot{\mu}^{2}+\dot{\nu}^{2}\right)+E d^{2}\left(\cosh { }^{2} \mu-\cos ^{2} \nu\right)\right.\right. \\
& \quad \int_{\mu(0)=\mu^{\prime}} \int_{(311)} \\
& \left.\left.\quad+\alpha d(\cosh \mu-\cos \nu)-\frac{\hbar^{2}}{2 M}\left(\frac{\lambda^{2}-\frac{1}{4}+k_{1}^{2} \cosh \mu}{\sinh ^{2} \mu}+\frac{\lambda^{2}-\frac{1}{4}+k_{1}^{2} \cos \nu}{\sin ^{2} \nu}\right)\right] d s\right\} \cdot
\end{aligned}
$$

Unfortunately we cannot solve this path integral.

3.3.8. We consider the potential $\left(\rho=\sqrt{x^{2}+y^{2}}\right)$

$$
V_{8}(\vec{x})=-\frac{\alpha}{\rho}+\sqrt{\frac{2}{\rho}}\left(\beta_{1} \cos \frac{\phi}{2}+\beta_{2} \sin \frac{\phi}{2}\right)+F(z) .
$$

This potential is the same potential as in 3.1.4 with an additional $z$-dependence via $F(z)$. The corresponding path integral formulations and solutions in mutually orthogonal circular parabolic coordinates are therefore a straightforward combination of the results of 3.1 .4 and $K_{F}\left(z^{\prime \prime}, z^{\prime} ; T\right)$.

\section{Summary.}

In the present work we have made the first step in the classification of all the presently possible path integral solutions of the Smorodinsky-Winternitz potentials in two and three dimensions. This approach as compared with the Schrödinger approach has advantages as well as disadvantages. The advantages are that the path integral gives a global view of the problem in question, whereas the Schrödinger equation only a local one. This property enables one to incorporate into the Feynman path integral many important features like topological effects, perturbation theory as well as the investigation of non-perturbative effects, a semi-classical expansion of the propagator, and many more. It is quite likely that the Feynman path integral is possibly the only consistent way to incorporate renormalization theory in a unified field theory. Therefore it is always important to investigate solvable quantum systems "from the point of view of fluctuating paths" [31]. A further advantage is that the explicit computation of the propagator (the Feynman kernel), respectively the (energy-dependent) Green's function, gives simultaneously the spectral expansion into the wave-functions and the energy spectrum.

A disadvantage is the fact that an explicit path integration can be done only in a limited number of coordinate systems, namely in polar, spherical, and some problems in parabolic coordinates, respectively coordinate systems which are related to them. Path integral evaluations 
in, say, elliptic, paraboloidal and other parametric coordinate systems are not accessible. One may say that one of the very advantages of the Feynman path integral, i.e. its global approach to a quantum mechanical problem, leads in these cases to a disadvantage, because the actual path integration would require "addition theorems" in terms of special functions of parametric coordinate systems, i.e. their corresponding spherical functions of the Laplace operator. Such "addition theorems" are not to our disposal presently. However, it seems reasonable that a path integral identity in conical coordinates can be derived via the path integral on the sphere $S^{(6)}$, where for the spherical functions a representation in terms of Jacobi functions is chosen.

Explicitly solvable models always involve solutions in terms of hypergeometric and confluent hypergeometric functions. We have seen that in at least one coordinate system each of the discussed Smorodinsky-Winternitz potentials can be solved in terms of these higher transcendental functions. In the parameter dependent coordinates the solution cannot be written in closed form. Therefore an investigation of the Feynman path integral in such coordinate systems is desirable and further studies along these lines will be subject to future work. This is not idle doing, then, for instance, the spheroidal coordinate system is only one into which the two-center problem separates. In future investigations we will try to discuss these more complicated problems.

The present work does not claim a full analysis of the quantum mechanical SmorodinskyWinternitz system. Such an analysis must include not only a comprehensive classification but also the determination of the interbasis coefficients of the wave-functions of the same energy. This requires the knowledge of the dynamical symmetry group which is responsible for the accidental degeneracy of the energy spectrum of a Smorodinsky-Winternitz potential.

In due time we will continue our work along the lines described above, and we will also try to include an analysis in which quantum mechanical problems in spaces of (positive and negative) constant curvature are taken into account. This will include a path integral approach of parametric coordinate systems on the sphere and pseudo-sphere, the study of the Coulomband the Higgs oscillator problem in particular, and of Smorodinsky-Winternitz potentials in spaces of constant curvature in general.

\section{Acknowledgement.}

G.S.Pogosyan would like to thank the members of the II.Institut für Theoretische Physik, Hamburg University, for their kind hospitality.

\section{Appendix.}

In this appendix we want to give a short discussion of the path integral problem of the anharmonic sextic oscillator as encountered in parabolic coordinates for the Holt potential. Let us consider the one-dimensional path integral

$$
K\left(x^{\prime \prime}, x^{\prime} ; T\right)=\int_{x\left(t^{\prime}\right)=x^{\prime}}^{x\left(t^{\prime \prime}\right)=x^{\prime \prime}} \mathcal{D} x(t) \exp \left\{\frac{\mathrm{i}}{\hbar} \int_{t^{\prime}}^{t^{\prime \prime}}\left[\frac{M}{2}\left(\dot{x}^{2}-\omega^{2} x^{6}\right)+\frac{\hbar^{2} k^{2}}{2 M} x^{2}-\frac{\hbar^{2} \beta^{2}}{2 M x^{2}}\right] d t\right\}
$$

Performing a combined space-time transformation with the new coordinate $z=x^{4}$, i.e. $x=$ $F(z)=z^{1 / 4}$, and the new "pseudo-time" defined by $s_{j}-s_{j-1}=\delta_{j}=16 x_{j}^{3} x_{j-1}^{3}\left(t_{j}-t_{j-1}\right)$ on the lattice, respectively $s^{\prime \prime}=16 \int_{t^{\prime}}^{t} x^{6}(s) d s$, we obtain the transformtion formulæ (c.f. [28, 40, 59, 61, 85, 110])

$$
K\left(x^{\prime \prime}, x^{\prime} ; T\right)=\int_{-\infty}^{\infty} \frac{d E}{2 \pi \mathrm{i}} \mathrm{e}^{-\mathrm{i} T E / \hbar} G\left(z^{\prime \prime}, z^{\prime} ; E\right)
$$




$$
G\left(z^{\prime \prime}, z^{\prime} ; E\right)=\left[F^{\prime}\left(z^{\prime \prime}\right) F^{\prime}\left(z^{\prime}\right)\right]^{1 / 2} \frac{\mathrm{i}}{\hbar} \int_{0}^{\infty} \hat{K}\left(z^{\prime \prime}, z^{\prime} ; s^{\prime \prime}\right) d s^{\prime \prime}
$$

and the kernel $\hat{K}\left(z^{\prime \prime}, z^{\prime} ; s^{\prime \prime}\right)$ is given by $\left[\lambda^{2}-\frac{1}{4}=\left(\beta^{2}+15 / 4\right) 16\right]$

$$
\hat{K}\left(z^{\prime \prime}, z^{\prime}, s^{\prime \prime}\right)=\int_{z(0)=z^{\prime}}^{z\left(s^{\prime \prime}\right)=z^{\prime \prime}} \mathcal{D} z(s) \exp \left\{\frac{\mathrm{i}}{\hbar} \int_{0}^{s^{\prime \prime}}\left[\frac{M}{2} \dot{z}^{2}+\frac{\hbar^{2} k^{2}}{32 M z}+\frac{E}{16 z^{3 / 2}}-\frac{\hbar^{2}}{2 M} \frac{\lambda^{2}-\frac{1}{4}}{z^{2}}\right] d s\right\} .
$$

We see that this path integral cannot be evaluated for $E \neq 0$. This corresponds in the case of the Holt potential (and all related ones as well) that we can only "solve" it for the parabolic separation parameter equal to zero.

If we now set $E=0$ the path integral $\hat{K}\left(s^{\prime \prime}\right)$ is actually a Coulomb potential problem. For the Coulomb potential the Feynman kernel is not known in closed form, however the corresponding Green's function $G^{(C)}(E)$ is. Insertion in (315) gives the the zero-energy Green's function of (313)

$$
\begin{aligned}
G\left(z^{\prime \prime}, z^{\prime} ; 0\right)= & {\left[F^{\prime}\left(z^{\prime \prime}\right) F^{\prime}\left(z^{\prime}\right)\right]^{1 / 2} G^{(C)}\left(z^{\prime \prime}, z^{\prime} ;-\frac{M \omega^{2}}{32}\right) } \\
= & \left(z^{\prime} z^{\prime \prime}\right)^{1 / 8} \frac{4}{\hbar \omega} \frac{\Gamma\left(\frac{1}{2}+\lambda+\hbar k^{2} / 8 M \omega\right)}{\Gamma(1+2 \lambda)} \\
& \quad \times W_{-\hbar k^{2} / 8 M \omega, \lambda}\left(\frac{M \omega}{2 \hbar} z_{>}\right) M_{-\hbar k^{2} / 8 M \omega, \lambda}\left(\frac{M \omega}{2 \hbar} z_{<}\right) .
\end{aligned}
$$

Note that this formula is a generalization of the result of Steiner [119] where only one power potential term was taken into account. It is obvious that in a similar way an $x^{14}$ potential term can be considered instead of a $x^{6}$, leading to a (zero-energy) Green's function of a radial harmonic oscillator. Furthermore, higher power potentials can be also treated by choosing another power dependence in the transformation $x=F(z)$.

Note that the zero-energy Green's function can also be evaluated in cases, where coordinate systems in flat space separate the Laplace equation (and not the Helmhotz, respectively the Schrödinger equation), i.e. coordinate systems which are R-separable, as for instance bispherical or toroidal coordinates. 


\section{References}

[1] Y.Aharonov and D.Bohm: Significance of Electromagnetic Potentials in the Quantum Theory; Phys.Rev. 115 (1959) 485

[2] A.O.Barut, A.Inomata and G.Junker: Path Integral Treatment of the Hydrogen Atom in a Curved Space of Constant Curvature; J.Phys.A: Math.Gen. 20 (1987) 6271

[3] A.O.Barut, A.Inomata and G.Junker: Path Integral Treatment of the Hydrogen Atom in a Curved Space of Constant Curvature II; J.Phys.A: Math.Gen. 23 (1990) 1179

[4] C.C.Bernido and A.Inomata: Path Integrals with a Periodic Constraint: The Aharonov-Bohm Effect; J.Math.Phys. 22 (1981) 715

[5] A.Bohm, Y.Ne'eman and A.O.Barut (eds.): Dynamical Groups and Spectrum Generating Algebras Vol.I (Academic Press, New York, 1987)

[6] M.Böhm and G.Junker: Path Integration Over Compact and Noncompact Rotation Groups; J.Math.Phys. 28 (1987) 1978

[7] D.Bonatos, C.Daskaloyannis and K.Kokkotas: Deformed Oscillator Algebras for Two-Dimensional Quantum Superintegrable Systems; Thessaloniki University preprint, THES-TP-10-93, hep-th/9309088, September 1993

[8] H.Buchholz: The Confluent Hypergeometric Function, Springer Tracts in Natural Philosophy, Volume 15 (Springer-Verlag, Berlin, 1969)

[9] F.Calogero: Solution of a Three-Body Problem in One Dimension; J.Math.Phys. 10 (1969) 2191

[10] M.V.Carpio-Bernido: Path Integral Quantization of Certain Noncentral Systems with Dynamical Symmetries; J.Math.Phys. 32 (1991) 1799

[11] M.V.Carpio-Bernido: Green Function for an Axially Symmetric Potential Field: A Path Integral Evaluation in Polar Coordinates; J.Phys.A: Math.Gen. 24 (1991) 3013

[12] M.V.Carpio-Bernido and C.C.Bernido: An Exact Solution of a Ring-Shaped Oscillator Plus a $c \sec ^{2} \theta / r^{2}$ Potential; Phys.Lett. A 134 (1989) 395; Algebraic Treatment of a Double Ring-Shaped Oscillator; Phys.Lett. A 137 (1989) 1

[13] M.V.Carpio-Bernido, C.C.Bernido and A.Inomata: Exact Path Integral Treatment of Two Classes of Axially Symmetric Potentials, in "Third International Conference on Path Integrals From meV to MeV", 1989, p.442, eds.: V.Sa-yakanit et al. (World Scientific, Singapore, 1989)

[14] M.V.Carpio-Bernido and A.Inomata: Path Integral Treatment of the Hartmann Potential; in "Bielefeld Encounters in Physics and Mathematics VII, Path Integrals From meV to MeV", 1985, p.261; eds.: M.C.Gutzwiller et al. (World Scientific, Singapore, 1986)

[15] D.P.L.Castrigiano and F.Stärk: New Aspects of the Path Integrational Treatment of the Coulomb Potential; J.Math.Phys. 30 (1989) 2785

[16] B.K.Cheng: Path-Integral Evaluation of the Propagator for a Charged Particle in a Constant Magnetic Field and with the Vector Potential of a Solenoid, Phys.Rev. A 36 (1987) 2964

[17] L.Chetouani, L.Guechi and T.F.Hammann: Exact Path Integral for the Ring Potential; Phys.Lett. A 125 (1987) 277; Algebraic Treatment of a General Noncentral Potential; J.Math.Phys. 33 (1992) 3410

[18] L.Chetouani, L.Guechi and T.F.Hammann: Exact Path Integral Solution of the Coulomb Plus Aharonov-Bohm Potential; J.Math.Phys. 30 (1989) 655

[19] L.Chetouani and T.F.Hammann: Coulomb Green's Function, in a $n$-Dimensional Euclidean Space; J.Math.Phys. 27 (1986) 2944

[20] L.Chetouani and T.F.Hammann: Traitement Exact des Système Coulombiens, dans le Formalisme des Intégrales de Feynman, en Coordonnées Parabolique; Nuovo Cimento B 98 (1987) 1 
[21] A.Cisneros and H.V.McIntosh: Symmetry of the Two-Dimensional Hydrogen Atom; J.Math.Phys. 10 (1969) 277

[22] C.A.Coulson and P.D.Robinson: Wave Functions for the Hydrogen Atom in Spheroidal Coordinates I: The Derivation and Properties of the Functions; Proc.Phys.Soc. 71 (1958) 815

[23] J.Daboul, P.Slodowy and C.Daboul: The Hydrogen Algebra as Centerless Twisted Kac-Moody Algebra; Ben Gurion University preprint, August 1993

[24] L.S.Davtyan, G.S.Pogosyan, A.N.Sissakian and V.M.Ter-Antonyan, Transformations Between Parabolic Bases of the Two-Dimensional Hydrogen Atom in the Continuous Spectrum; Theor. Math.Phys. 74 (1988) 157; Two-Dimensional Hydrogen Atom. Reciprocal Expansions of the Polar and Parabolic Bases of the Continuous Spectrum; Theor.Math.Phys. 66 (1986) 146

[25] B.S.DeWitt: Dynamical Theory in Curved Spaces. I. A Review of the Classical and Quantum Action Principles; Rev.Mod.Phys. 29 (1957) 377

[26] J.S.Dowker: When is the 'Sum Over Classical Paths' Exact? J.Phys.A: Gen.Phys. 3 (1970) 451

[27] J.S.Dowker: Quantum Mechanics on Group Space and Huygens' Principle; Ann.Phys.(N.Y.) 62 (1971) 361

[28] I.H.Duru: Path Integrals Over SU(2) Manifold and Related Potentials; Phys.Rev. D 30 (1984) 2121

[29] I.H.Duru: On the Path Integral for the Potential $V=a r^{-2}+b r^{2}$; Phys.Lett. A 112 (1985) 421

[30] I.H.Duru and H.Kleinert: Solution of the Path Integral for the H-Atom; Phys.Lett. B 84 (1979) 185

[31] I.H.Duru and H.Kleinert: Quantum Mechanics of H-Atoms From Path Integrals; Fortschr.Phys. 30 (1982) 401

[32] L.P.Eisenhart: Enumeration of Potentials for Which One-Particle Schroedinger Equations Are Separable; Phys.Rev. 74 (1948) 87

[33] A.Erdélyi, W.Magnus, F.Oberhettinger and F.G.Tricomi (eds.): Higher Transcendental Functions, Vol.III, $\quad$ p.272 (McGraw Hill, New York, 1955) //TCPSMTDH JOB 90099099,CLASS=X,TIME=(,1),MSGCLASS=Z //*MAIN SYSTEM $=$ JGLOBAL,RELPRI $=$ HIG,LINES $=5000$, USER $=$ TCPSMT

// EXEC PGM=IEBGENER //SYSIN DD DUMMY //SYSPRINT DD SYSOUT $=* / /$ SYSUT2 DD SYSOUT=(B,TCPSMT) //SYSUT1 DD DATA,DLM=@@ HELO DSYIBM.DESY.DE NOOP MAIL FROM: ¡I02GRO@DSYIBM.DESY.DE; RCPT TO: ¡hep-th@xxx.lanl.govi DATA

[34] A.Erdélyi, W.Magnus, F.Oberhettinger and F.G.Tricomi (eds.): Tables of Integral Transforms, Vol.II (McGraw Hill, New York, 1955)

[35] N.W.Evans: Superintegrability in Classical Mechanics; Phys.Rev. A 41 (1990) 5666; Group Theory of the Smorodinsky-Winternitz System; J.Math.Phys. 32 (1991) 3369

[36] N.W.Evans: Super-Integrability of the Winternitz System; Phys.Lett. A 147 (1990) 483

[37] R.P.Feynman: Space-Time Approach to Non-Relativistic Quantum Mechanics; Rev.Mod.Phys. 20 (1948) 367

[38] R.P.Feynman and A.Hibbs: Quantum Mechanics and Path Integrals (McGraw Hill, New York, 1965)

[39] V.Fock: Zur Theorie des Wasserstoffatoms; Zeitschr.Phys. 98 (1935) 145, reprinted in [5], p.400

[40] W.Fischer, H.Leschke and P.Müller: Changing Dimension and Time: Two Well-Founded and Practical Techniques for Path Integration in Quantum Physics; J.Phys.A: Math.Gen. 25 (1992) 3835

[41] W.Fischer, H.Leschke and P.Müller: Path Integration in Quantum Physics by Changing the Drift of the Underlying Diffusion Process: Application of Legendre Processes; Ann.Phys.(N.Y.) 227 (1993) 206

[42] J.Friš, V.Mandrosov, Ya.A.Smorodinsky, M.Uhlir and P.Winternitz: On Higher Symmetries in Quantum Mechanics; Phys.Lett. 16 (1965) 354,

J.Friš, Ya.A.Smorodinskiǐ, M.Uhlî̌ and P.Winternitz: Symmetry Groups in Classical and Quantum Mechanics; Sov.J.Nucl.Phys. 4 (1967) 444 
[43] I.M.Gel'fand and M.I.Graev: Geometry of Homogeneous Spaces, Representations of Groups in Homogeneous Spaces and Related Questions. I; Amer.Math.Soc.Transl., Ser.2 37 (1964) 351

[44] I.M.Gel'fand, M.I.Graev and N.Ya.Vilenkin: Generalized Functions, Vol.5 (Academic Press, New York, 1966)

[45] C.C.Gerry and V.A.Singh: Feynman Path-Integral Approach to the Aharonov-Bohm Effect; Phys.Rev. D 20 (1979) 2550;

[46] C.C.Gerry: Dynamical Group for a Ring Potential; Phys.Lett. A 118 (1986) 445

[47] J.-L.Gervais and A.Jevicki: Point Canonical Transformations in the Path Integral; Nucl.Phys. B $110(1976) 93$

[48] M.J.Goovaerts: Path-Integral Evaluation of a Nonstationary Calogero Model; J.Math.Phys. 16 (1975) 720

[49] I.S.Gradshteyn and I.M.Ryzhik: Table of Integrals, Series, and Products (Academic Press, New York, 1980)

[50] Ya.A.Granovsky, A.S.Zhedanov and I.M.Lutzenko: Quadratic Alegbra as a 'Hidden' Symmetry of the Hartmann Potential; J.Phys.A: Math.Gen. 24 (1991) 3887

[51] Ya.A.Granovsky, A.S.Zhedanov and I.M.Lutzenko: Quadratic Algebras and Dynamics in Curved Spaces. I. Oscillator; Theor.Math.Phys. 91 (1992) 474; Quadratic Algebras and Dynamics in Curved Spaces. II. The Kepler Problem; Theor.Math.Phys. 91 (1992) 604

[52] C.Grosche: The Product Form for Path Integrals on Curved Manifolds; Phys.Lett. A 128 (1988) 113

[53] C.Grosche: Path Integral Solution of a Class of Potentials Related to the Pöschl-Teller Potential; J.Phys.A: Math.Gen. 22 (1989) 5073

[54] C.Grosche: The Path Integral for the Kepler Problem on the Pseudosphere; Ann.Phys.(N.Y.) 204 (1990) 208

[55] C.Grosche: Separation of Variables in Path Integrals and Path Integral Solution of Two Potentials on the Poincaré Upper Half-Plane; J.Phys.A: Math.Gen. 23 (1990) 4885

[56] C.Grosche: Coulomb Potentials by Path-Integration; Fortschr.Phys. 40 (1992) 695

[57] C.Grosche: Path Integral Solution of Two Potentials Related to the $\mathrm{SO}(2,1)$ Dynamical Algebra; J.Phys.A: Math.Gen. 26 (1993) L279

[58] C.Grosche: Path Integration and Separation of Variables in Spaces of Constant Curvature in Two and Three Dimensions; DESY preprint, DESY 93-141, hep-th/9311001, October 1993,

[59] C.Grosche and F.Steiner: Path Integrals on Curved Manifolds; Zeitschr.Phys. C 36 (1987) 699

[60] C.Grosche and F.Steiner: The Path Integral on the Pseudosphere; Ann.Phys.(N.Y.) 182 (1988) 120

[61] C.Grosche and F.Steiner: Table of Feynman Path Integrals; to appear in: Springer Tracts in Modern Physics; Classification of Exactly Solvable Feynman Path Integrals; in Proceedings of the "Fourth International Conference on Path Integrals from meV to $\mathrm{MeV}$ ", Tutzing, Germany 1992, p.276; H.Grabert, A.Inomata, L.S.Schulman and U.Weiss (eds.): World Scientific, Singapore, 1993).

[62] A.Guha and S.Mukherjee: Exact Solution of the Schrödinger Equation with Noncentral Parabolic Potentials; J.Math.Phys. 28 (1987) 840

[63] H.Hartmann: Bewegung eines Körpers in einem ringförmigen Potentialfeld; Theoret.Chim.Acta 24 (1972) 201

[64] J.Hietarinta: Direct Methods for the Search of the Second Invariant; Phys.Rep. 147 (1987) 87

[65] P.W.Higgs: Dynamical Symmetries in a Spherical Geometry; J.Phys.A: Math.Gen. 12 (1979) 309

[66] C.R.Holt: Construction of New Integrable Hamiltonians in Two Degrees of Freedom; J.Math.Phys. 23 (1982) 1037

[67] L.Infeld: On a New Treatment of Some Eigenvalue Problems; Phys.Rev. 59 (1941) 737 
[68] A.Inomata: Exact Path-Integration for the Two Dimensional Coulomb Problem; Phys.Lett. A 87 (1982) 387

[69] A.Inomata: Alternative Exact-Path-Integral-Treatment of the Hydrogen Atom; Phys.Lett. A 101 (1984) 253

[70] A.Inomata: Roles of Dynamical Groups in Path Integration; to appear in the proceedings of the "International Workshop on 'Symmetry Methods in Physics' in Memory of Prof. Ya. A. Smorodinsky", Dubna, July 1993

[71] A.Inomata, H.Kuratsuji and C.C.Gerry: Path Integrals and Coherent States of SU(2) and SU(1,1) (World Scientific, Singapore, 1992)

[72] A.Inomata and R.Wilson: Path Integral Realization of a Dynamical Group; Lecture Notes in Physics 261, p.42 (Springer-Verlag, Berlin, 1985).

[73] W.Kallies, I.Lukach, G.S.Pogosyan and A.N.Sissakian: Ellipsoidal Bases for Harmonic Oscillator; to appear in the proceedings of the "International Workshop on 'Symmetry Methods in Physics' in Memory of Prof. Ya. A. Smorodinsky", Dubna, July 1993

[74] E.G.Kalnins: Separation of Variables for Riemannian Spaces of Constant Curvature (Longman Scientific \& Technical, Essex, 1986)

[75] E.G.Kalnins, W.Miller Jr. and P.Winternitz: The Group $O(4)$, Separation of Variables and the Hydrogen Atom; SIAM J.Appl.Math. 30 (1976) 630

[76] M.Kibler and C.Campigotto: On a Generalized Aharonov-Bohm Plus Oscillator System; Phys.Lett. A 181 (1993) 1; Classical and Quantum Study of a Generalized Kepler-Coulomb System; Int.J.Quantum Chem. 45 (1993) 209

[77] M.Kibler, G.-H.Lamot and P.Winternitz: Classical Trajectories for Two Ring-Shaped Potentials; Int.J.Quantum Chem. 43 (1992) 625

[78] M.Kibler, L.G.Mardoyan and G.S.Pogosyan: On a Generalized Kepler-Coulomb Problem System: Interbasis Expansion; Lyon preprint, January 1994

[79] M.Kibler and T.Negadi: Motion of a Particle in a Ring-Shaped Potential: An Approach Via a Nonbijective Canonical Transformation; Int.J.Quantum Chem. 26 (1984) 405

[80] M.Kibler and T.Negadi: Motion of a Particle in a Coulomb Field Plus Aharonov-Bohm Potential; Phys.Lett. A 124 (1987) 42

[81] M.Kibler and P.Winternitz: Dynamical Invariance Algebra of the Hartmann Potential; J.Phys.A: Math.Gen. 20 (1987) 4097

[82] M.Kibler and P.Winternitz: Periodicity and Quasi-Periodicity for Super-Integrable Hamiltonian Systems; Phys.Lett. A 147 (1990) 338

[83] H.M.Kiefer and D.M.Fradkin: Comments on Separability Operators, Invariance Ladder Operators, and Quantization of the Kepler Problem in Prolate-Spheroidal Coordinates; J.Math.Phys. 9 (1968) 627

[84] H.Kleinert: How to do the Time Sliced Path Integral for the H Atom; Phys.Lett. A 120 (1987) 361

[85] H.Kleinert: Path Integrals in Quantum Mechanics, Statistics, and Polymer Physics (World Scientific, Singapore, 1990)

[86] W.Langguth and A.Inomata: Remarks on the Hamiltonian Path Integral in Polar Coordinates; J.Math.Phys. 20 (1979) 499

[87] H.I.Leemon: Dynamical Symmetries in a Spherical Geometry; J.Phys.A: Math.Gen. A 12 (1979) 489

[88] Le Van Hoang and N.T.Giang: On the Green Function for a Hydrogen-Like Atom in the Dirac Monopole Field Plus the Aharonov-Bohm Field; J.Phys.A: Math.Gen. 26 (1993) 3333

[89] M.W.Lucht and P.D.Jarvis: Quasiexactly Solvable Problems in the Path-Integral Formalism; Phys.Rev. A 47 (1993) 817

[90] I.V.Lutsenko, G.S.Pogosyan, A.N.Sissakian and V.M.Ter-Antonyan: Hydrogen Atom as Indicator of Hidden Symmetry of a Ring-Shaped Potential; Theor.Math.Phys. 83 (1990) 633; 
[91] I.V.Lutsenko, L.G.Mardoyan, G.S.Pogosyan and A.N.Sissakian: The Generalization of Interbasis Oscillator Expansions "Cylinder-Sphere" in the Field of a Rings-Shaped Potential; Communications JINR, P2-89-814, Dubna, 1989

[92] D.W.McLaughlin and L.S.Schulman: Path Integrals in Curved Spaces; J.Math.Phys. 12 (1971) 2520

[93] A.A.Makarov, Ya.A.Smorodinsky, Kh.Valiev and P.Winternitz: A Systematic Search for Nonrelativistic Systems with Dynamical Symmetries; Nuovo Cimento A 52 (1967) 1061

[94] L.G.Mardoyan, G.S.Pogosyan, A.N.Sissakian and V.M.Ter-Antonyan: Spheroidal Analysis of Hydrogen Atom; J.Phys.A: Math.Gen. 16 (1983) 711; Spheroidal Corrections to the Spherical and Parabolic Bases of the Hydrogen Atom; Theor.Math.Phys. 64 (1985) 762; Elliptic Basis for a Circular Oscillator; Theor.Math.Phys. 65 (1985) 1113

[95] L.G.Mardoyan, G.S.Pogosyan, A.N.Sissakian and V.M.Ter-Antonyan: Two-Dimensional Hydrogen Atom: I. Elliptic Bases; Theor.Math.Phys. 61 (1984) 1021; Hidden Symmetry, Separation of Variables and Interbasis Expansions in the Two-Dimensional Hydrogen Atom; J.Phys.A: Math.Gen. 18 (1985) 455; Interbasis Expansion in a Two-Dimensional Hydrogen Atom; Theor.Math.Phys. 63 (1985) 406; To the Elliptic Base of a Two-Dimensional Hydrogen Atom; Preprint JINR, P-84-110, Dubna 1984

[96] M.S.Marinov and M.V.Terentyev: A Functional Integral on Unitary Groups; Sov.J.Nucl.Phys. 28 (1978) 729; Dynamics on the Group Manifold and Path Integral; Fortschr.Phys. 27 (1979) 511

[97] J.Meixner and F.W.Schäfke: Mathieusche Funktionen und Sphäroidfunktionen (Springer-Verlag, Berlin, 1954)

[98] M.M.Mizrahi: The Weyl Correspondence and Path Integrals; J.Math.Phys. 16 (1975) 2201

[99] M.M.Mizrahi: Phase Space Path Integrals, without Limiting Procedure; J.Math.Phys. 19 (1978) 298

[100] M.M.Mizrahi: Correspondence Rules and Path Integrals; Nuovo Cimento B 61 (1981) 81

[101] P.Moon and D.E.Spencer: Field Theory Handbook (Springer-Verlag, Berlin, 1988)

[102] C.Morette: On the Definition and Approximation of Feynman's Path Integrals; Phys.Rev. 81 (1951) 848

[103] C.Morette-DeWitt: The Semiclassical Expansion; Ann.Phys.(N.Y.) 97 (1976) 367

[104] P.M.Morse: Diatomic Molecules According to the Wave Mechanics II. Vibrational Levels; Phys.Rev. 34 (1929), 57.

[105] P.M.Morse and H.Feshbach: Methods of Theoretical Physics (McGraw-Hill, New York, 1953)

[106] M.N.Olevskiĩ: Triorthogonal Systems in Spaces of Constant Curvature in which the Equation $\Delta_{2} u+\lambda u=0$ Allows the Complete Separation of Variables; Math.Sb. 27 (1950) 379 (in russian)

[107] M.Omote: Point Canonical Transformations and the Path Integral; Nucl.Phys. B 120 (1977) 325

[108] V.S.Otchik and V.M.Red'kov: Quantum Mechanical Kepler Problem in Space with Constant Curvature; Minsk 1983, preprint No. 298 (in russian)

[109] K.Pak and I.Sökmen: A New Exact Path Integral Treatment of the Coulomb and the Morse Potential Problems; Phys.Lett. A 100 (1984) 327

[110] N.K.Pak and I.Sökmen: General New-Time Formalism in the Path Integral; Phys.Rev. A 30 (1984) 1629

[111] J.Patera and P.Winternitz: A New Basis for the Representation of the Rotation Group. Lamé and Heun Polynomials; J.Math.Phys. 14 (1973) 1130

[112] W.Pauli: Über das Wasserstoffspektrum vom Standpunkt der neuen Quantenmechanik; Zeitschr. Phys. 36 (1926) 336, reprinted in [5], p.371

[113] D.Peak and A.Inomata: Summation Over Feynman Histories in Polar Coordinates; J.Math.Phys. $10(1969) 1422$ 
[114] R.F.Picken: The Propagator for Quantum Mechanics on a Group Manifold from an Infinite Dimensional Analogue of the Duistermaat-Heckman Integration Formula; J.Phys.A: Math.Gen. 22 (1989) 2285

[115] G.S.Pogosyan and A.N.Sissakian and S.I.Vinitsky: Higgs Oscillator in Three-Dimensional Space with Positive Constant Curvature. Interbasis Expansion; to appear in the proceedings of the "International Workshop on 'Symmetry Methods in Physics' in Memory of Prof. Ya. A. Smorodinsky", Dubna, July 1993

[116] C.Quesne: A New Ring-Shaped Potential and its Dynamical Invariance Algebra; J.Phys.A: Math.Gen. 21 (1988) 3093

[117] I.Sökmen: Exact Path-Integral Solution for a Charged Particle in a Coulomb Plus Aharonov-Bohm Potential; Phys.Lett. A 132 (1988) 65

[118] F.Steiner: Exact Path Integral Treatment of the Hydrogen Atom; Phys.Lett. A 106 (1984) 363

[119] F.Steiner: Path Integrals in Polar Coordinates From eV to GeV; in "Bielefeld Encounters in Physics and Mathematics VII, Path Integrals From meV to MeV", 1985, p.335, eds.: M.C.Gutzwiller et al. (World Scientific, Singapore, 1986)

[120] A.F.Steveson: Note on the "Kepler Problem" in a Spherical Space, and the Factorization Method of Solving Eigenvalue Problems; Phys.Rev. 59 (1941) 842

[121] E.Schrödinger: A Method of Determining Quantum-Mechanical Eigenvalues and Eigenfunctions; Proc.Roy.Irish Soc. 46 (1941) 9; Further Studies on Solving Eigenvalue Problems by Factorization; Proc.Roy.Irish Soc. 46 (1941) 183; The Factorization of the Hypergeometric Equation; Proc.Roy.Irish Soc. 47 (1941) 53

[122] S.N.Storchak: Remarks on Quantization of the Hydrogen Atom by the Path-Integral Method; Theor.Math.Phys. 82 (1990) 32

[123] V.Tanikella and A.Inomata: Path Integral Calculation of Entanglement Probabilities for Bound Macromolecules; Phys.Lett. A 87 (1982) 196

[124] E.Teller: Über das Wasserstoffmolekülion; Zeitschr.Phys. 61 (1930), 458.

[125] A.N.Vaidya and H.Boschi-Filho: Algebraic Calculation of the Green's Function of the Hartmann Potential; J.Math.Phys. 31 (1990) 1951

[126] N.Ja.Vilenkin: Special Functions Connected with Class 1 Representations of Groups of Motions in Spaces of Constant Curvature; Trans.Moscow Math.Soc. (1963) 209

[127] F.W.Wiegel: Path Integral Methods in Statistical Mechanics; Phys.Rep. C 16 (1975) 57

[128] P.Winternitz: Group Theoretical Methods in Physics, p.549; Konf. Montreal 1976; eds.: R.T.Sharp and B.Kolman (Academic Press, New York, 1977)

[129] P.Winternitz, L.Lukac and Ya.A.Smorodinsky: Quantum Mechanics of the Little Group of the Poincaré Group; Sov.J.Nucl.Phys. 7 (1968) 139

[130] B.Zaslow and M.E.Zandler: Two-Dimensional Analog of the Hydrogen Atom; Amer.J.Phys. 35 (1967) 1118

[131] A.S.Zhedanov: Hidden Symmetry Algebra and Overlap Coefficients for Two Ring-Shaped Potentials; J.Phys.A: Math.Gen. 26 (1993) 4633 